High order non-conforming multi-element Discontinuous Galerkin method for time domain electromagnetics

Clément Durochat, Stéphane Lanteri , Claire Scheid

RESEARCH

REPORT

$\mathrm{N}^{\circ} 8257$

March 2013

Project-Team Nachos 



\title{
inzián
}

\section{High order non-conforming multi-element Discontinuous Galerkin method for time domain electromagnetics}

\author{
Clément Durochat ${ }^{*}$, Stéphane Lanteri *, Claire Scheid ${ }^{*}$ \\ Project-Team Nachos \\ Research Report n 8257 - March 2013 - 52 pages
}

\begin{abstract}
We study a high order Discontinuous Galerkin Time Domain (DGTD) method for solving the system of Maxwell equations. This method is formulated on a non-conforming multielement mesh mixing an unstructured triangulation for the discretization of irregularly shaped objects with a structured (Cartesian) quadrangulation for the rest of the computational domain. Within each element, the electromagnetic field components are approximated by a high order nodal polynomial. The DG method proposed here makes use of a centered scheme for the definition of the numerical traces of the electric and magnetic fields at element interfaces, associated to a second order or fourth order leap-frog scheme for the time integration of the associated semi-discrete equations. We formulate this DGTD method in 3D and study its theoretical properties. In particular, an a priori convergence estimation is elaborated. Finally, we present numerical results for the application of the method to $2 \mathrm{D}$ test problems.
\end{abstract}

Key-words: computational electromagnetics, time domain Maxwell equations, discontinuous Galerkin method, multi-element mesh, non-conforming mesh

* INRIA Sophia Antipolis-Méditerranée

$\dagger$ University of Nice-Sophia Antipolis 


\section{Méthode Galerkin discontinue en maillage multi-éléments non-conforme pour les équations de Maxwell en domaine temporel}

Résumé : On étudie une méthode Galerkin discontinue en domaine temporel pour la résolution numérique du système des équations de Maxwell instationnaires. Cette méthode est formulée sur un maillage multi-élément non-conforme associant une triangulation non-structurée pour la discrétisation d'objets aux formes irrégulières, et une quadrangulation structurée du reste du domaine de calcul. Dans chaque élément du maillage, les composantes du champ éléctromagnétique sont approchées par une interpolation polynomiale d'ordre élevé. La formulation Galerkin discontinue adoptée ici utilise un schéma centré pour la définition des traces numériques des champs électrique et magnétique aux interfaces entre éléments voisins, et un schéma saute-mouton du second ordre ou du quatrième ordre pour l'intégration en temps des équations semi-discrétisées. Cette méthode est présentée dans un cadre 3D et on étudie ses propriétés théoriques. En particulier, on réalise une étude de convergence a priori. Enfin, on présente des résultats numériques de l'application de la méthode à des problèmes tests $2 \mathrm{D}$.

Mots-clés : électromagnétisme numérique, équations de Maxwell, domaine temporel, méthode Galerkin discontinue, maillage multi-éléments, maillage non-confoorme. 


\section{Introduction}

Nowadays, a variety of modeling strategies exist for the computer simulation of electromagnetic wave propagation in the time domain. In particular, the ability to deal accurately and in a flexible way with complex geometries has been extensively studied in the context of unstructured mesh based discretization methods as alternatives to the well established FDTD (Finite Difference Time Domain) method [24]. In the FDTD method, the whole computational domain is discretized using a structured (Cartesian) grid. This greatly simplifies the discretization process but also represents the main limitation of the method when complicated geometrical objects come into play. Besides, the last 10 years have witnessed an increased interest in so-called DGTD (Discontinuous Galerkin Time Domain) methods. Thanks to the use of discontinuous finite element spaces, DGTD methods can easily handle elements of various types and shapes, irregular non-conforming meshes, and even locally varying polynomial degree, and hence offer great flexibility in the mesh design. They also lead to (block-) diagonal mass matrices and therefore yield fully explicit, inherently parallel methods when coupled with explicit time stepping. Moreover, continuity is weakly enforced across mesh interfaces by adding suitable bilinear forms (often referred as numerical fluxes) to the standard variational formulations. DGTD methods which have been developed on quadrangular (2D case) or hexahedral (3D case) meshes [3]- 16], as well as on triangular or tetrahedral [13]- [9]-[7]-[5] meshes. DGTD methods have now acquired a sufficient level of maturity and have successfully penetrated several scientific and technological communities following to their adaptation to increasingly complex modeling contexts such as [5]-17]-23]-14] among others. Worthwhile to note, the method has also been adopted for the first time in a commercial software as the time domain alternative of a very well known electromagnetic wave simulation tool 22. In all the previously mentioned works on DGTD methods, the first order (or mixed) form of the system of time domain Maxwell equations is considered and, within each mesh element, the electromagnetic field components are approximated by a arbitrarily high order nodal polynomial.

One of the main features of DGTD methods is their enhanced flexibility with regards to the type of meshes they can deal with. Indeed, DGTD methods can easily handle irregular possibly non-conforming meshes, constituted of elements of various types and shapes. Thus, not surprisingly, several attempts have been made to combine time domain methods based on structured meshes with DGTD formulations on unstructured meshes. A low order solution strategy in this direction is presented in 8] in the form of a combination of FDTD and FVTD (that can be seen as the lowest order form of a DGTD method). A hybridization of FDTD and DGTD is described in [10]-11] for 2D problems. The main goal is to accurately model (with FVTD or DGTD) the geometric details of a curved objects, while maintaining the simplicity and the speed of FDTD for the surrounding space. The proposed strategy relies on a simple interfacing scheme which is deduced from a particular setting of FDTD as a particular case of DGTD and vice versa. An intermediate layer of quadrangular elements is introduced between the Cartesian FDTD grid and the triangular DGTD mesh. Within this layer, the coupling of the two schemes is facilitated by regarding FDTD as a 0th order DGTD method. The DGTD method makes use of central fluxes and both methods are time advanced using a second order leap-frog scheme. A high order hybrid strategy has been recently studied in [4] which combines a spectral FETD method on quadrangular meshes with a DGTD method on triangular meshes. The coupling of the two methods is achieved by using an upwind numerical flux on the internal boundary defining the interface between the quadrangular and triangular meshes. In both methods, the semi-discrete equations are time advanced using a 4th order Runge-Kutta scheme. The accuracy of the resulting high order hybrid FETD-DGTD method is clearly demonstrated for several 2D test problems involving different scattering geometries but there is no assessment of the computational performances 
in comparison to a solution strategy based on fully triangular meshes. A different and somewhat less general hybridization approach is proposed in [21. This scheme combines the widely adopted Finite Integration Technique (FIT) with a Finite Volume Method (FVM) based either on central or upwind fluxes. However, in this combined FIT-FVM method, a single mesh type is used, more precisely a Cartesian grid, and the hybridization strategy relies on the longitudinal-transverse splitting of the discrete curl operator. Then, the FIT discretizes the transverse two-dimensional subproblem while the FVM discretizes the longitudinal one-dimensional subproblem. The resulting FIT-FVM strategy aims at being applied to propagation problems where the size of the computational domain along one preferred direction is electrically much larger than along the others. By proceeding in that way, the superior dispersive properties of the FVM are exploited along one direction.

In this work, we are concerned with the possibility of relying on a single discretization scheme, i.e. a discontinuous Galerkin method, and improving the efficiency of the simulation by employing a hybrid structured-unstructured mesh made of orthogonal quadrangular elements (hexahedral elements in 3D) for the discretization of the regular part of the computational domain and triangular elements (tetrahedral elements in 3D) for the discretization of the irregularly shaped objects of the propagation scene. To fully exploit the flexibility of a discontinuous Galerkin method with regards to the discretization of the computational domain, we allow hanging nodes at the interface between the structured and unstructured parts of the mesh. In addition, we consider the possibility of using different approximation orders in these regions. Of particular interest to the present study is the work discussed in 12 . In this paper, the authors describe a high order in space and time DGTD method formulated on non-conforming hybrid meshes for the simulation of seismic wave propagation in the 2D case. This DGTD method is based on the ADER (Arbitrarily high-order DERivatives) scheme for achieving high order accuracy in time, while the adopted DG formulation makes use of an upwind flux for the evaluation of the boundary integral term at an interface (edge) shared by neighboring elements. In the presence of hanging nodes resulting from the use of non-conforming meshes, this boundary integral term is evaluated thanks to an appropriate Gauss-Legendre numerical quadrature formula. A similar approach is adopted in the present work however the DG formulation adopted here is an extension of the one introduced in [9] i.e. it is a based on a central flux and the time integration relies on a leap-frog scheme. Note that we consider both a second order and a fourth order leap-frog scheme in order to assess the impact of a higher accuracy in time. More recently, an interior penalty DGTD method formulated on non-conforming tetrahedral meshes for the solution of the 3D Maxwell equations has been presented in [19. The development of the method was motivated by integrated circuit (IC) packaging applications for which it is very difficult to construct a conforming mesh. Instead, for the complicated geometries involved in such applications, a subdomain-based meshing approach is particularly attractive and allowing hanging nodes is highly desirable in this context. The authors also demonstrate the benefits of using a local time strategy for dealing with the strong variations in elements size when meshing IC structures. In addition, they discuss about appropriate parallelization and load balancing strategies for further improving the overall computational efficiency of the proposed numerical methodology.

We formulate a non-conforming multi-element DGTD- $\mathbb{P}_{p} \mathbb{Q}_{k}$ method in the $3 \mathrm{D}$ case and study its theoretical properties. In particular, an a priori convergence estimation is elaborated. The method is then evaluated numerically thanks to its implementation in the 2D case and its application to several test problems. The rest of the paper is organized as follows: the initial and boundary value problem to be solved is briefly outlined in section 2 section 3 is devoted to the formulation of the DGTD- $\mathbb{P}_{p} \mathbb{Q}_{k}$ method on hybrid hexahdral-tetrahedral meshes; the convergence of this method is analyzed in section 4 numerical results for $2 \mathrm{D}$ propagation problems are presented in section 5. finally, section 6 summarizes the main result of this study. 


\section{The continuous problem}

Let $\Omega$ be an opened, bounded domain of $\mathbb{R}^{3}$ with boundary $\Gamma$. The system of 3D Maxwell equations is given by:

$$
\left\{\begin{aligned}
\epsilon \frac{\partial \mathbf{E}}{\partial t}-\operatorname{rot}(\mathbf{H}) & =-z_{0} \sigma \mathbf{E} \\
\mu \frac{\partial \mathbf{H}}{\partial t}+\operatorname{rot}(\mathbf{E}) & =0
\end{aligned}\right.
$$

where $\mathbf{x}=\left(x_{1}, x_{2}, x_{3}\right)^{\mathrm{T}}$ while $\mathbf{E}(\mathbf{x}, t)=\left(E_{x}(\mathbf{x}, t), E_{y}(\mathbf{x}, t), E_{z}(\mathbf{x}, t)\right)^{\mathrm{T}}$ and

$\mathbf{H}(\mathbf{x}, t)=\left(H_{x}(\mathbf{x}, t), H_{y}(\mathbf{x}, t), H_{z}(\mathbf{x}, t)\right)^{\mathrm{T}}$ respectively denote the electric and magnetic fields; $\epsilon \equiv \epsilon(\mathbf{x}), \mu \equiv \mu(\mathbf{x})$ and $\sigma \equiv \sigma(\mathbf{x})$ respectively stand for the electric permittivity, the magnetic permeability and the electric conductivity. Equations (1) have been normalized such that $\epsilon$ and $\mu$ define relative quantities $\left(z_{0}=\sqrt{\mu_{0} / \epsilon_{0}}\right.$ is the vacuum impedance). Our goal is to solve system (1) in a domain $\Omega$ with boundary $\partial \Omega=\Gamma_{a} \cup \Gamma_{m}$, where we impose the following boundary conditions: $\mathbf{n} \times \mathbf{E}=0$ on $\Gamma_{m}$, and $\mathcal{L}(\mathbf{E}, \mathbf{H})=\mathcal{L}\left(\mathbf{E}_{\text {inc }}, \mathbf{H}_{\text {inc }}\right)$ on $\Gamma_{a}$ where $\mathcal{L}(\mathbf{X}, \mathbf{Y})=\mathbf{n} \times \mathbf{X}-z \mathbf{n} \times(\mathbf{Y} \times \mathbf{n})$ with $z=\sqrt{\mu / \varepsilon}$. Here $\mathbf{n}$ denotes the unit outward normal to $\partial \Omega$ and $\left(\mathbf{E}_{\text {inc }}, \mathbf{H}_{\text {inc }}\right)$ is a given incident field. The first boundary condition is called metallic (referring to a perfectly electric conducting surface) while the second condition is an absorbing boundary condition, more precisely the Silver-Müller condition which is a first order approximation of the exact absorbing boundary condition. This absorbing condition is applied on $\Gamma_{a}$ which represents an artificial truncation of the computational domain. To simplify the presentation in the following, we assume $\sigma=0$ and rewrite system (1) under a vectorial form (also referred to as pseudo-conservative form) where $\mathbf{W}=(\mathbf{E}, \mathbf{H})^{\mathrm{T}} \in \mathbb{R}^{6}$ :

$$
Q\left(\partial_{t} \mathbf{W}\right)+\nabla \cdot F(\mathbf{W})=0 \text { with } \nabla=(\partial / \partial x, \partial / \partial y, \partial / \partial z)^{\mathrm{T}},
$$

where:

$$
Q=\left[\begin{array}{cc}
\epsilon \mathrm{I}_{3 \times 3} & 0_{3 \times 3} \\
0_{3 \times 3} & \mu \mathrm{I}_{3 \times 3}
\end{array}\right], \quad \mathbf{W}=\left(\begin{array}{c}
\mathbf{E} \\
\mathbf{H}
\end{array}\right) \quad \text { and } \quad \mathbf{J}=\left(\begin{array}{c}
-z_{0} \sigma \mathbf{E} \\
0 \\
0 \\
0
\end{array}\right)
$$

and:

$$
\nabla \cdot F(\mathbf{W})=\partial_{x_{1}}\left(F_{1}(\mathbf{W})\right)+\partial_{x_{2}}\left(F_{2}(\mathbf{W})\right)+\partial_{x_{3}}\left(F_{3}(\mathbf{W})\right) \in \mathbb{R}^{6}
$$

where:

$$
\begin{aligned}
& F_{1}(\mathbf{W})=\left[\begin{array}{cc}
0_{3 \times 3} & N_{1} \\
-N_{1} & 0_{3 \times 3}
\end{array}\right] \mathbf{W}=\left(0, H_{3},-H_{2}, 0,-E_{3}, E_{2}\right)^{\mathrm{T}}, \\
& F_{2}(\mathbf{W})=\left[\begin{array}{cc}
0_{3 \times 3} & N_{2} \\
-N_{2} & 0_{3 \times 3}
\end{array}\right] \mathbf{W}=\left(-H_{3}, 0, H_{1}, E_{3}, 0,-E_{1}\right)^{\mathrm{T}}, \\
& F_{3}(\mathbf{W})=\left[\begin{array}{cc}
0_{3 \times 3} & N_{3} \\
-N_{3} & 0_{3 \times 3}
\end{array}\right] \mathbf{W}=\left(H_{2},-H_{1}, 0,-E_{2}, E_{1}, 0\right)^{\mathrm{T}},
\end{aligned}
$$

are vectors $\in \mathbb{R}^{6}$ and $N_{1}, N_{2}, N_{3}$ are the (skew-symmetric) matrices given by

$$
N_{1}=\left[\begin{array}{ccc}
0 & 0 & 0 \\
0 & 0 & 1 \\
0 & -1 & 0
\end{array}\right] \quad, \quad N_{2}=\left[\begin{array}{ccc}
0 & 0 & -1 \\
0 & 0 & 0 \\
1 & 0 & 0
\end{array}\right] \quad, \quad N_{3}=\left[\begin{array}{ccc}
0 & 1 & 0 \\
-1 & 0 & 0 \\
0 & 0 & 0
\end{array}\right]
$$

$\mathrm{RR} \mathrm{n}^{\circ} 8257$ 


\section{DGTD method formulation}

This section is concerned with the numerical treatment of system 21 using a DGTD method. The method is here formulated in the 3D case although, in this preliminary study, we present in section 5 numerical results for the solution of $2 \mathrm{D}$ problems. When appropriate, we comment on specific differences between the $2 \mathrm{D}$ and $3 \mathrm{D}$ situations.

\subsection{Discretization in space}

We assume that the domain $\Omega$ is discretized as $\mathscr{C}_{h}=\bigcup_{i=1}^{N} c_{i}=\mathscr{T}_{h} \bigcup \mathscr{Q}_{h}$ where the $c_{i}$ 's are hexahedral $\left(\in \mathscr{Q}_{h}\right)$ and tetrahedral $\left(\in \mathscr{T}_{h}\right)$ elements. The resulting mesh is hybrid and nonconforming (i.e. with hanging nodes on a common face between two elements with different types) (see [6]-[7]). In this study, we assume a certain kind of non-conformity as shown Fig. 1 , i.e. we limit ourselves to configurations such that for a given non-conforming interface, the triangular faces on one side are obtained by subdividing the quadrangular face on the other side. In practice, we allow for an arbitrary refinement level $l$ of the triangular faces $(l=1$ on Fig. 1$)$. $\Gamma$ denotes the boundary of the domain $\Omega$ and $\Gamma_{m}$ (resp. $\Gamma_{a}$ ) the part of the boundary where the field verifies a metallic boundary condition (resp. an absorbing boundary condition).
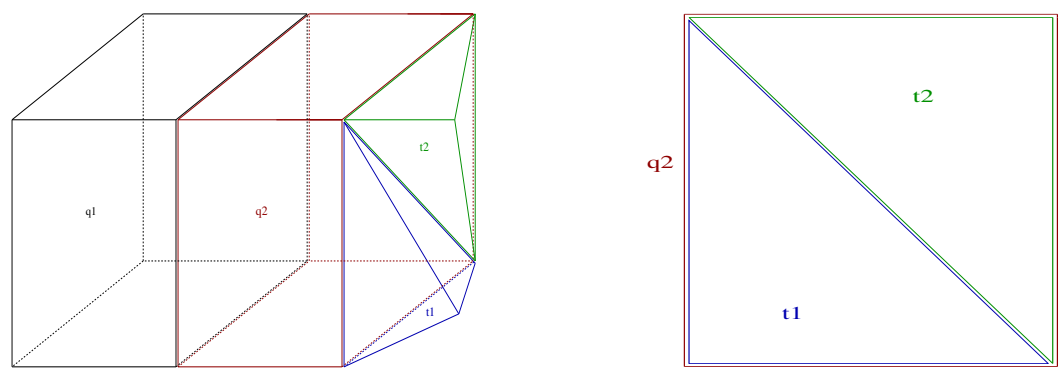

Figure 1: Left: type of non-conformity considered in 3D, between a hexadron (q2) and two tetrahedra ( $\mathrm{t} 1$ and $\mathrm{t} 2$ ) - Right: $2 \mathrm{D}$ view of the non-conforming hybrid face between $\mathrm{q} 2$ and, $\mathrm{t} 1$ and $\mathrm{t} 2$

\subsubsection{Weak formulation}

Let $\vec{\psi}=\left(\psi_{1}, \psi_{2}, \psi_{3}, \psi_{4}, \psi_{5}, \psi_{6}\right)^{\mathrm{T}} \in \mathbb{R}^{6}$ be a vectorial test function. Applying the scalar product by $\langle.,$.$\rangle byr \vec{\psi}$ to system $(2)$ and integrating over $c_{i}$ we obtain the starting point of the weak formulation of the problem:

$$
\int_{c_{i}}\left\langle Q\left(\partial_{t} \mathbf{W}\right), \vec{\psi}\right\rangle d \mathbf{x}+\int_{c_{i}}\langle\nabla \cdot F(\mathbf{W}), \vec{\psi}\rangle d \mathbf{x}=0
$$

In the sequel, to simplify the presentation of the discrete formulation, we assume that $\Gamma_{a}=\emptyset$ but we note that the discrete formulations readily extends to the situation where $\Gamma_{a} \neq \emptyset$. We introduce $\mathbb{P}_{p}\left[c_{i}\right]$ the space of scalar polynomial functions with degree at most $p$ in $c_{i} \in \mathscr{T}_{h}$, and $\mathbb{Q}_{k}\left[c_{i}\right]$ the space of scalar polynomial functions with degree at most $k$ with respect to each 
space variable separately in $c_{i} \in \mathscr{Q}_{h}$. The discrete solution vector $\mathbf{W}_{h}$ is searched for in the approximation space $V_{h}^{6}$ defined by:

$$
V_{h}=\left\{\begin{array}{l|l}
v_{h} \in L^{2}(\Omega) & \begin{array}{l}
\forall c_{i} \in \mathscr{T}_{h}, v_{h \mid c_{i}} \in \mathbb{P}_{p}\left[c_{i}\right] \\
\forall c_{i} \in \mathscr{Q}_{h}, v_{h \mid c_{i}} \in \mathbb{Q}_{k}\left[c_{i}\right]
\end{array}
\end{array}\right\}
$$

Let $\phi_{i}=\left(\varphi_{i 1}, \varphi_{i 2}, \cdots, \varphi_{i d_{i}}\right)^{\mathrm{T}}$ be a local basis of $\mathbb{P}_{p}\left[c_{i}\right]$ with $d_{i}$ the dimension of the basis with regards to the interpolation order of the physical field in the tetrahedron $c_{i}$, and $\theta_{i}=$ $\left(\vartheta_{i 1}, \cdots, \vartheta_{i b_{i}}\right)^{\mathrm{T}}$ a local basis of $\mathbb{Q}_{k}\left[c_{i}\right]$ with $b_{i}$ the dimension of the basis with regards to the interpolation order of the physical field in the hexahedron $c_{i}$. The local degrees of freedom are denoted by $\mathbf{W}_{i l}=\left(\mathbf{E}_{i l}, \mathbf{H}_{i l}\right)^{\mathrm{T}} \in \mathbb{R}^{6}$ while $\mathbf{W}_{i}=\left(\mathbf{E}_{i}, \mathbf{H}_{i}\right)^{\mathrm{T}} \in \mathbb{R}^{6}$ is the restriction of the approximate solution to the cell $c_{i}\left(\left.\mathbf{W}_{h}\right|_{c_{i}}\right)$. We then have that:

$$
\begin{aligned}
\text { - If } c_{i} \in \mathscr{T}_{h}: \mathbf{W}_{i}(\mathbf{x}) & =\sum_{l=1}^{d_{i}} \mathbf{W}_{i l} \varphi_{i l}(\mathbf{x})=\left(\begin{array}{c}
\sum_{l=1}^{d_{i}} \mathbf{E}_{i l} \varphi_{i l}(\mathbf{x}) \\
\sum_{l=1}^{d_{i}} \mathbf{H}_{i l} \varphi_{i l}(\mathbf{x})
\end{array}\right) \\
& =\left(\begin{array}{c}
\mathbf{E}_{i}(\mathbf{x}) \\
\mathbf{H}_{i}(\mathbf{x})
\end{array}\right) \text { and } \mathbb{P}_{p}\left[c_{i}\right]=\operatorname{Vect}\left(\varphi_{i 1}, \ldots, \varphi_{i d_{i}}\right) . \\
\text { - If } c_{i} \in \mathscr{Q}_{h}: \mathbf{W}_{i}(\mathbf{x}) & =\sum_{l=1}^{b_{i}} \mathbf{W}_{i l} \vartheta_{i l}(\mathbf{x})=\left(\begin{array}{c}
\sum_{l=1}^{b_{i}} \mathbf{E}_{i l} \vartheta_{i l}(\mathbf{x}) \\
\sum_{l=1}^{b_{i}} \mathbf{H}_{i l} \vartheta_{i l}(\mathbf{x}) \\
\sum_{l=1}
\end{array}\right) \\
& =\left(\begin{array}{c}
\mathbf{E}_{i}(\mathbf{x}) \\
\mathbf{H}_{i}(\mathbf{x})
\end{array}\right) \text { and } \mathbb{Q}_{k}\left[c_{i}\right]=\operatorname{Vect}\left(\vartheta_{i 1}, \ldots, \vartheta_{i b_{i}}\right) .
\end{aligned}
$$

Let $a_{i j}=c_{i} \cap c_{j}\left(a_{i j}\right.$ is possibly empty) be the common interface between $c_{i}$ and $c_{j}$ and let us denote by $\mathcal{V}_{i}=\left\{j \mid c_{i} \cap c_{j} \neq \varnothing\right\}$ the set of neighboring cells of $c_{i}$. We also introduce $\breve{\mathbf{n}}_{i j}$ which is the unitary normal vector on $a_{i j}$ directed from $c_{i}$ to $c_{j}$, and $\breve{\mathbf{n}}_{i j}=\left(\breve{n}_{i j}^{1}, \breve{n}_{i j}^{2}, \breve{n}_{i j}^{3}\right)^{\mathrm{T}} \in \mathbb{R}^{3}$. Since the approximate fields $\mathbf{E}_{h}$ and $\mathbf{H}_{h}$ are allowed to be discontinuous across element boundaries, a specific treatment must be introduced when evaluating such a field at a cell boundary. In the context of finite volume methods, this leads to the notion of numerical flux. In this study, we choose to use a centered approximation for evaluating $\left.\mathbf{W}_{h}\right|_{a_{i j}}$, i.e. $\forall i, \forall j \in \mathcal{V}_{i}$ we set $\mathbf{W}_{h \mid a_{i j}} \approx\left(\left.\mathbf{W}_{i}\right|_{a_{i j}}+\left.\mathbf{W}_{j}\right|_{a_{i j}}\right) / 2$. For the boundary cells i.e. for interfaces located on the discretization of $\Gamma$, we consider that $c_{j}$ is a fictitious cell and $\mathbf{W}_{j}$ is defined according to the boundary conditions that are set on $\Gamma$. For instance, if $a_{i j} \in \partial \Omega^{m}$ we set:

$$
\mathbf{W}_{j}=\left[\begin{array}{cc}
-I_{3 \times 3} & 0_{3 \times 3} \\
0_{3 \times 3} & I_{3 \times 3}
\end{array}\right] \mathbf{W}_{i} \text {, i.e. : }\left(\begin{array}{c}
\mathbf{E}_{j} \\
\mathbf{H}_{j}
\end{array}\right)=\left(\begin{array}{r}
-\mathbf{E}_{i} \\
\mathbf{H}_{i}
\end{array}\right) \text {. }
$$

From now on, we consider two cases referred a (A) and (B) in the following.

Case (A) corresponds to the situation where $c_{i} \in \mathscr{T}_{h}$ is a tetrahedron. Then, $\forall j \in \mathcal{V}_{i}, a_{i j}$ is either a boundary interface (i.e. $a_{i j} \in \mathscr{T}_{m}^{i} \subset \Gamma_{m}$ or $a_{i j} \in \mathscr{T}_{a}^{i} \subset \Gamma_{a}$ ), or an interface between two neighboring tetrahedra (i.e. $a_{i j} \in \mathscr{T}_{d}^{i}$ ), or an interface between a tetrahedron and an hexahedron (i.e. $\quad a_{i j} \in \mathscr{H}_{d}^{i}$ ) which will be referred as a hybrid interface. Injecting $\mathbf{W}_{i}$ in $(3)$, integrating by by parts twice (not detailed here) and using the defintion of the numerical flux, we obtain the 
the weak formulation:

$$
\begin{aligned}
& 2 Q_{i} \int_{c_{i}}\left\langle\partial_{t} \mathbf{W}_{i}, \vec{\psi}\right\rangle d \mathbf{x}+ \\
& \int_{c_{i}}\left(\left\langle\sum_{k=1}^{3} \partial_{x_{k}} \mathcal{N}^{k} \mathbf{W}_{i}, \vec{\psi}\right\rangle-\sum_{k=1}^{3}\left\langle\partial_{x_{k}} \vec{\psi}, \mathcal{N}^{k} \mathbf{W}_{i}\right\rangle\right) d \mathbf{x}+ \\
& \sum_{a_{i j} \in \mathscr{T}_{d}^{i}} \int_{a_{i j}}^{k}\left\langle\mathcal{M}_{i j} \mathbf{W}_{j}, \vec{\psi}\right\rangle d \sigma+\sum_{a_{i j} \in \mathscr{T}_{m}^{i}} \int_{a_{i j}}\left\langle\mathcal{S}_{i m} \mathbf{W}_{i}, \vec{\psi}\right\rangle d \sigma+ \\
& \sum_{a_{i j} \in \mathscr{H}_{d}^{i}} \int_{a_{i j}}\left\langle\mathcal{M}_{i j} \mathbf{W}_{j}, \vec{\psi}\right\rangle d \sigma=0,
\end{aligned}
$$

with:

- $Q_{i}=\left[\begin{array}{cc}\epsilon_{i} I_{3 \times 3} & 0_{3 \times 3} \\ 0_{3 \times 3} & \mu_{i} I_{3 \times 3}\end{array}\right]$ where $\epsilon_{i}$ and $\mu_{i}$ have constant values on $c_{i}$,

- $\mathcal{N}^{k}=\left[\begin{array}{cc}0_{3 \times 3} & N_{k} \\ -N_{k} & 0_{3 \times 3}\end{array}\right]$,

- $\mathcal{M}_{i j}=\sum_{k=1}^{3} \breve{n}_{i j}^{k} \mathcal{N}^{k}=\left[\begin{array}{cc}0_{3 \times 3} & \breve{N}_{i j} \\ -\breve{N}_{i j} & 0_{3 \times 3}\end{array}\right]$

where $\breve{N}_{i j}=\sum_{k=1}^{3} \breve{n}_{i j}^{k} N_{k}=\left[\begin{array}{ccc}0 & \breve{n}_{i j}^{3} & -\breve{n}_{i j}^{2} \\ -\breve{n}_{i j}^{3} & 0 & \breve{n}_{i j}^{1} \\ \breve{n}_{i j}^{2} & -\breve{n}_{i j}^{1} & 0\end{array}\right]$,

- $\mathcal{S}_{i m}=\left[\begin{array}{cc}0_{3 \times 3} & \breve{N}_{i m} \\ \breve{N}_{i m} & 0_{3 \times 3}\end{array}\right]$.

Remark 1. The following comments can be made:

- In each term of (7) $\mathbf{W}_{i} \in \mathbb{P}_{p}\left[c_{i}\right]$ (respectively $\mathbf{W}_{j} \in \mathbb{P}_{p}\left[c_{j}\right]$ ), except for faces $\in \mathscr{H}_{d}^{i}$ for which $\mathbf{W}_{j} \in \mathbb{Q}_{k}\left[c_{j}\right]$.

- $\forall \mathbf{X} \in \mathbb{R}^{3}$, we have $\breve{N}_{i j} \mathbf{X}=\mathbf{X} \times \breve{\mathbf{n}}_{i j}$. In addition $\breve{\mathbf{n}}_{i j}=-\breve{\mathbf{n}}_{j i}$ and thus $\breve{N}_{i j}=-\breve{N}_{j i}$.

- The matrices $N_{k}$ and $\breve{N}_{i j}$ have size $3 \times 3$ and are skew-symmetric; the matrices $\mathcal{N}^{k}$ and $\mathcal{M}_{i j}$ have size $6 \times 6$ and are symmetric; $\mathcal{S}_{i m}$ is a $6 \times 6$ skew-symmetric matrix.

Case (B) corresponds to the situation where $c_{i} \in \mathscr{Q}_{h}$ is a hexahedron. Then, $\forall j \in \mathcal{V}_{i}, a_{i j}$ is either a boundary interface (i.e. $a_{i j} \in \mathscr{Q}_{m}^{i} \subset \Gamma_{m}$ or $a_{i j} \in \mathscr{Q}_{a}^{i} \subset \Gamma_{a}$ ), or an interface between two neighboring hexahedra (i.e. $a_{i j} \in \mathscr{Q}_{d}^{i}$ ), or a hybrid interface (i.e. $a_{i j} \in \mathscr{H}_{d}^{i}$ ) which will be referred as a hybrid interface. Proceeding similarly as done for case (A), we obtain:

$$
\begin{gathered}
2 Q_{i} \int_{c_{i}}\left\langle\partial_{t} \mathbf{W}_{i}, \vec{\psi}\right\rangle d \mathbf{x}+ \\
\int_{c_{i}}\left(\left\langle\sum_{k=1}^{3} \partial_{x_{k}} \mathcal{N}^{k} \mathbf{W}_{i}, \vec{\psi}\right\rangle-\sum_{k=1}^{3}\left\langle\partial_{x_{k}} \vec{\psi}, \mathcal{N}^{k} \mathbf{W}_{i}\right\rangle\right) d \mathbf{x}+ \\
\sum_{a_{i j} \in \mathscr{Q}_{d}^{i}} \int_{a_{i j}}\left\langle\mathcal{M}_{i j} \mathbf{W}_{j}, \vec{\psi}\right\rangle d \sigma+\sum_{a_{i j} \in \mathscr{Q}_{m}^{i}} \int_{a_{i j}}\left\langle\mathcal{S}_{i m} \mathbf{W}_{i}, \vec{\psi}\right\rangle d \sigma+ \\
\sum_{a_{i j} \in \mathscr{H}_{d}^{i}} \int_{a_{i j}}\left\langle\mathcal{M}_{i j} \mathbf{W}_{j}, \vec{\psi}\right\rangle d \sigma=0 .
\end{gathered}
$$


Remark 2. Here, in each term $\mathbf{W}_{i} \in \mathbb{Q}_{k}\left[c_{i}\right]$ (respectively $\mathbf{W}_{j} \in \mathbb{Q}_{k}\left[c_{j}\right]$ ), except for faces $\in \mathscr{H}_{d}^{i}$ for which $\mathbf{W}_{j} \in \mathbb{P}_{p}\left[c_{j}\right]$.

\subsubsection{Semi-discrete equations}

We now introduce the notations:

$$
\begin{gathered}
\Phi_{i}=\int_{c_{i}} \phi_{i} \phi_{i}^{\mathrm{T}} d \mathbf{x}, \quad \Phi_{i}^{x_{k}}=\int_{c_{i}}\left(\phi_{i}\left(\partial_{x_{k}} \phi_{i}\right)^{\mathrm{T}}-\left(\partial_{x_{k}} \phi_{i}\right) \phi_{i}^{\mathrm{T}}\right) d \mathbf{x}, \\
\Phi_{i j}=\int_{a_{i j}} \phi_{i} \phi_{j}^{\mathrm{T}} d \sigma \text { and } \Upsilon_{i j}=\int_{a_{i j}} \phi_{i} \theta_{j}^{\mathrm{T}} d \sigma,
\end{gathered}
$$

where:

- $\phi_{i}$ is a column vector $\in \mathbb{R}^{d_{i}}$ and $\phi_{i}^{\mathrm{T}}$ (respectively $\phi_{j}^{\mathrm{T}}$ ) is a row vector $\in \mathbb{R}^{d_{i}}$ (respectively de $\left.\mathbb{R}^{d_{j}}\right)$.

- $\theta_{j}^{\mathrm{T}}$ is a row vector $\in \mathbb{R}^{b_{j}}$.

- $\Phi_{i}$ is a $d_{i} \times d_{i}$ symmetric positive definite matrix and $\Phi_{i}^{x_{k}}$ is a $d_{i} \times d_{i}$ skew-symmetric matrix.

- $\Phi_{i j}$ is a $d_{i} \times d_{j}$ symmetric positive matrix.

- $\Upsilon_{i j}$ is a $d_{i} \times b_{j}$ rectangular matrix.

Let $\overline{\mathbf{E}}_{i}$ and $\overline{\mathbf{H}}_{i}$ the vectors of local degrees of freedom (which are both in $\mathbb{R}^{3 d_{i}}$ ), $\mathbf{E}_{i l}$ and $\mathbf{H}_{i l}$ (which are both in $\mathbb{R}^{3}$ ) for $l=1, \ldots, d_{i}$, associated to the tetrahedron $c_{i}$; similarly, let $\widetilde{\mathbf{E}}_{j}$ and $\widetilde{\mathbf{H}}_{j}$ the vectors of local degrees of freedom (which are both in $\mathbb{R}^{3 b_{i}}$ ), $\mathbf{E}_{j l}$ and $\mathbf{H}_{j l}$ for $l=1, \ldots, b_{j}$, associated to the hexahedron $c_{i}$ :

$$
\overline{\mathbf{E}}_{i}=\left(\begin{array}{c}
\mathbf{E}_{i 1} \\
\vdots \\
\mathbf{E}_{i d_{i}}
\end{array}\right), \overline{\mathbf{H}}_{i}=\left(\begin{array}{c}
\mathbf{H}_{i 1} \\
\vdots \\
\mathbf{H}_{i d_{i}}
\end{array}\right), \widetilde{\mathbf{E}}_{j}=\left(\begin{array}{c}
\mathbf{E}_{j 1} \\
\vdots \\
\mathbf{E}_{j b_{j}}
\end{array}\right), \widetilde{\mathbf{H}}_{j}=\left(\begin{array}{c}
\mathbf{H}_{j 1} \\
\vdots \\
\mathbf{H}_{j b_{j}}
\end{array}\right) .
$$

Finally, we introduce the $6 d_{i}$ vectorial basis functions $\vec{\varphi}_{i 1}, \ldots, \vec{\varphi}_{i\left(6 d_{i}\right)}$ belonging to $\mathbb{R}^{6}$ whose 
elements are the scalar basis functions $\varphi_{i 1}, \ldots, \varphi_{i d_{i}}$ of $\mathbb{P}_{p_{i}}$ :

$$
\begin{aligned}
\vec{\varphi}_{i 1} & =\left(\begin{array}{c}
\varphi_{i 1} \\
0 \\
0 \\
0 \\
0 \\
0
\end{array}\right), \vec{\varphi}_{i 2}=\left(\begin{array}{c}
0 \\
\varphi_{i 1} \\
0 \\
0 \\
0 \\
0
\end{array}\right), \quad \ldots, \vec{\varphi}_{i 6}=\left(\begin{array}{c}
0 \\
0 \\
0 \\
0 \\
0 \\
\varphi_{i 1} \\
0 \\
0 \\
0 \\
0 \\
0 \\
\varphi_{i 2} \\
0 \\
0 \\
0 \\
0
\end{array}\right), \vec{\varphi}_{i 8}=\left(\begin{array}{c}
\varphi_{i 2} \\
0 \\
0 \\
0 \\
0
\end{array}\right), \quad \ldots, \vec{\varphi}_{i 12}=\left(\begin{array}{c}
\varphi_{i 7} \\
\vdots \\
\varphi_{i d_{i}} \\
0 \\
0 \\
0 \\
0
\end{array}\right), \vec{\varphi}_{i\left(6 d_{i}-4\right)}=\left(\begin{array}{c}
0 \\
\varphi_{i d_{i}} \\
0 \\
0 \\
0 \\
0 \\
\vec{\varphi}_{i\left(6 d_{i}-5\right)}
\end{array}\right), \quad \ldots, \vec{\varphi}_{i\left(6 d_{i}\right)}=\left(\begin{array}{c}
0 \\
0 \\
0 \\
0 \\
0 \\
\varphi_{i d_{i}}
\end{array}\right) .
\end{aligned}
$$

In order to obtain the $6 d_{i}$ semi-discrete equations associated to each tetrahedron $c_{i}$ we perform several operations in the weak form (7). In the integrals over $c_{i}$ and $a_{i j}$ for $a_{i j} \in \mathscr{T}_{d}^{i}$ and $a_{i j} \in \mathscr{T}_{m}^{i}$, we replace $\mathbf{W}_{i}$ (respectively $\mathbf{W}_{j}$ ) by its expression (5) in the basis $\phi_{i}$ (respectively $\phi_{j}$ ) of $\mathbb{P}_{p_{i}}\left[c_{i}\right]$ (respectively $\mathbb{P}_{p_{j}}\left[c_{j}\right]$ ). For the integral in the summation over the faces of $\mathscr{H}_{d}^{i}$ we replace $\mathbf{W}_{j}$ by its expression (6) in the basis $\theta_{j}$ of $\mathbb{Q}_{k_{j}}\left[c_{j}\right]$. Finally, we replace $\vec{\psi}$ by $\vec{\varphi}_{i l}$ for $l=1, \ldots, 6 d_{i}$. We then obtain the local system of ordinary differential equations:

$$
\begin{aligned}
& 2 \mathcal{X}_{\mu, i} \frac{d \overline{\mathbf{H}}_{i}}{d t}-\sum_{k=1}^{3} \mathcal{X}_{i}^{x_{k}} \overline{\mathbf{E}}_{i}-\sum_{a_{i j} \in \mathscr{T}_{d}^{i}} \mathcal{X}_{i j} \overline{\mathbf{E}}_{j}+\sum_{a_{i j} \in \mathscr{T}_{m}^{i}} \mathcal{X}_{i m} \overline{\mathbf{E}}_{i}-\sum_{a_{i j} \in \mathcal{H}_{d}^{i}} \mathcal{A}_{i j} \widetilde{\mathbf{E}}_{j}=0, \\
& 2 \mathcal{X}_{\epsilon, i} \frac{d \overline{\mathbf{E}}_{i}}{d t}+\sum_{k=1}^{3} \mathcal{X}_{i}^{x_{k}} \overline{\mathbf{H}}_{i}+\sum_{a_{i j} \in \mathscr{T}_{d}^{i}} \mathcal{X}_{i j} \overline{\mathbf{H}}_{j}+\sum_{a_{i j} \in \mathscr{T}_{m}^{i}} \mathcal{X}_{i m} \overline{\mathbf{H}}_{i}+\sum_{a_{i j} \in \mathscr{H}_{d}^{i}} \mathcal{A}_{i j} \widetilde{\mathbf{H}}_{j}=0,
\end{aligned}
$$

where $\mathcal{X}_{\epsilon, i}$ and $\mathcal{X}_{\mu, i}$ are local mass matrices (i.e. involving terms of the form $\int_{\tau_{i}} \phi_{i}^{\mathrm{T}} \phi_{i} d \mathbf{x}$ ). Similarly, $\mathcal{X}_{i}^{x_{k}}$ is a matrix involving terms of the form $\int_{\tau_{i}}\left(\phi_{i}^{\mathrm{T}}\left(\partial_{x_{k}} \phi_{i}\right)-\left(\partial_{x_{k}} \phi_{i}\right)^{\mathrm{T}} \phi_{i}\right) d \mathbf{x}$, while $\mathcal{X}_{i j}$ and $\mathcal{X}_{i m}$ are matrices associated to boundary integral terms (i.e. involving terms of the form $\left.\int_{a_{i j}} \phi_{i}^{\mathrm{T}} \phi_{j} d \sigma\right)$. All these matrices are of size $3 d_{i} \times 3 d_{i}$ except $\mathcal{A}_{i j}$ whose size is $3 d_{i} \times 3 b_{j}$ (i.e. involving terms of the form $\int_{a_{i j}} \phi_{i}^{\mathrm{T}} \theta_{j} d \sigma$, with $a_{i j} \in \mathscr{H}_{d}^{i}$ ).

For the hexahedral cells we introduce:

$$
\Theta_{i}=\int_{c_{i}} \theta_{i} \theta_{i}^{\mathrm{T}} d \mathbf{x}, \quad \Theta_{i}^{x_{k}}=\int_{c_{i}}\left(\theta_{i}\left(\partial_{x_{k}} \theta_{i}\right)^{\mathrm{T}}-\left(\partial_{x_{k}} \theta_{i}\right) \theta_{i}^{\mathrm{T}}\right) d \mathbf{x}, \quad \Theta_{i j}=\int_{a_{i j}} \theta_{i} \theta_{j}^{\mathrm{T}} d \sigma,
$$


and we recall that:

$$
\Upsilon_{j i}^{\mathrm{T}}=\int_{a_{i j}} \theta_{i} \phi_{j}^{\mathrm{T}} d \sigma
$$

where:

- $\theta_{i}$ is a column vector in $\mathbb{R}^{b_{i}}$ and $\theta_{i}^{\mathrm{T}}$ (respectively $\theta_{j}^{\mathrm{T}}$ ) are row vectors in $\mathbb{R}^{b_{i}}$ (respectively $\left.\in \mathbb{R}^{b_{j}}\right) ; \phi_{j}^{\mathrm{T}}$ is a row vector $\in \mathbb{R}^{d_{j}}$.

- $\Theta_{i}$ is a $b_{i} \times b_{i}$ symmetric positive definite matrix, $\Theta_{i}^{x_{k}}$ a $b_{i} \times b_{i}$ skew-symmetric matrix and $\Theta_{i j}$ a $b_{i} \times b_{j}$ symmetric positive matrix.

- $\Upsilon_{j i}^{\mathrm{T}}$ is a $b_{i} \times d_{j}$ rectangular matrix.

Proceeding as previously, we formulate the local system of $6 b_{i}$ ordinary differential equations for each hexahedral cell:

$$
\begin{aligned}
& 2 \mathcal{W}_{\mu, i} \frac{d \widetilde{\mathbf{H}}_{i}}{d t}-\sum_{k=1}^{3} \mathcal{W}_{i}^{x_{k}} \widetilde{\mathbf{E}}_{i}-\sum_{a_{i j} \in \mathscr{Q}_{d}^{i}} \mathcal{W}_{i j} \widetilde{\mathbf{E}}_{j}+\sum_{a_{i j} \in \mathscr{Q}_{m}^{i}} \mathcal{W}_{i m} \widetilde{\mathbf{E}}_{i}-\sum_{a_{i j} \in \mathscr{H}_{d}^{i}} \mathcal{B}_{i j} \overline{\mathbf{E}}_{j}=0, \\
& 2 \mathcal{W}_{\epsilon, i} \frac{d \widetilde{\mathbf{E}}_{i}}{d t}+\sum_{k=1}^{3} \mathcal{W}_{i}^{x_{k}} \widetilde{\mathbf{H}}_{i}+\sum_{a_{i j} \in \mathscr{Q}_{d}^{i}} \mathcal{W}_{i j} \widetilde{\mathbf{H}}_{j}+\sum_{a_{i j} \in \mathscr{Q}_{m}^{i}} \mathcal{W}_{i m} \widetilde{\mathbf{H}}_{i}+\sum_{a_{i j} \in \mathscr{H}_{d}^{i}} \mathcal{B}_{i j} \overline{\mathbf{H}}_{j}=0,
\end{aligned}
$$

where the involved matrices are defined analagously to those characterizing the system of semidiscrete equations corresponding to case (A).

\subsection{Time integration}

We rewrite the systems of ordinary differential equations 10 and 111 as:

$$
\text { Case (A) : }\left\{\begin{array}{l}
2 \mathcal{X}_{\mu, i} \frac{d \overline{\mathbf{H}}_{i}}{d t}=\mathbf{A}_{\mathbf{E}, i}, \\
2 \mathcal{X}_{\epsilon, i} \frac{d \overline{\mathbf{E}}_{i}}{d t}=\mathbf{A}_{\mathbf{H}, i} .
\end{array} \text { Case (B) }:\left\{\begin{array}{l}
2 \mathcal{W}_{\mu, i} \frac{d \widetilde{\mathbf{H}}_{i}}{d t}=\mathbf{B}_{\mathbf{E}, i}, \\
2 \mathcal{W}_{\epsilon, i} \frac{d \widetilde{\mathbf{E}}_{i}}{d t}=\mathbf{B}_{\mathbf{H}, i} .
\end{array}\right.\right.
$$

Let:

$$
\begin{aligned}
S_{h}^{e l}:\left(V_{h}\right)^{3} & \rightarrow\left(V_{h}\right)^{3} \\
\mathbf{E} & \mapsto S_{h}^{\text {el }}(\mathbf{E}),
\end{aligned}
$$

and:

$$
\begin{aligned}
& S_{h}^{\text {mag }}:\left(V_{h}\right)^{3} \rightarrow\left(V_{h}\right)^{3} \\
& \mathbf{H} \mapsto S_{h}^{\text {mag }}(\mathbf{H}) \text {, }
\end{aligned}
$$

that are uniquely given by their degrees of freedom. We have:

$$
\begin{aligned}
& \text { Case (A) : } \begin{cases}\left(\overline{S_{h}^{e l}(\mathbf{E})}\right)_{i} & =\frac{1}{2}\left(\mathcal{X}_{\mu, i}\right)^{-1} \mathbf{A}_{\mathbf{E}, i}, \\
\left(\overline{S_{h}^{\text {mag }}(\mathbf{H})}\right)_{i} & =\frac{1}{2}\left(\mathcal{X}_{\epsilon, i}\right)^{-1} \mathbf{A}_{\mathbf{H}, i},\end{cases} \\
& \text { Case (B) : } \begin{cases}\left(\widetilde{\left.S_{h}^{e l}(\mathbf{E})\right)_{i}}\right. & =\frac{1}{2}\left(\mathcal{W}_{\mu, i}\right)^{-1} \mathbf{B}_{\mathbf{E}, i}, \\
\left.\widetilde{S_{h}^{\text {mag }}(\mathbf{H})}\right)_{i} & =\frac{1}{2}\left(\mathcal{W}_{\epsilon, i}\right)^{-1} \mathbf{B}_{\mathbf{H}, i} .\end{cases}
\end{aligned}
$$


Remark 3. With this notation:

$$
S_{h}^{e l}\left(\mathbf{E}_{h}\right)=\frac{\partial H_{h}}{\partial t} \quad \operatorname{and} S_{h}^{m a g}\left(\mathbf{H}_{h}\right)=\frac{\partial \mathbf{E}_{h}}{\partial t} .
$$

Time integration of systems 12 can be achieved by:

- A second order leap-frog scheme (LF2):

$$
\begin{aligned}
& \text { Case (A) : }\left\{\begin{array}{l}
\overline{\mathbf{H}}_{i}^{n+\frac{1}{2}}=\overline{\mathbf{H}}_{i}^{n-\frac{1}{2}}+\frac{\Delta t}{2}\left[\mathcal{X}_{\mu, i}\right]^{-1} \mathbf{A}_{\mathbf{E}, i}^{n}, \\
\overline{\mathbf{E}}_{i}^{n+1}=\overline{\mathbf{E}}_{i}^{n}+\frac{\Delta t}{2}\left[\mathcal{X}_{\epsilon, i}\right]^{-1} \mathbf{A}_{\mathbf{H}, i}^{n+\frac{1}{2}} .
\end{array}\right. \\
& \text { Case (B) : }\left\{\begin{array}{l}
\widetilde{\mathbf{H}}_{i}^{n+\frac{1}{2}}=\widetilde{\mathbf{H}}_{i}^{n-\frac{1}{2}}+\frac{\Delta t}{2}\left[\mathcal{W}_{\mu, i}\right]^{-1} \mathbf{B}_{\mathbf{E}, i}^{n}, \\
\widetilde{\mathbf{E}}_{i}^{n+1}=\widetilde{\mathbf{E}}_{i}^{n}+\frac{\Delta t}{2}\left[\mathcal{W}_{\epsilon, i}\right]^{-1} \mathbf{B}_{\mathbf{H}, i}^{n+\frac{1}{2}} .
\end{array}\right.
\end{aligned}
$$

The resulting method will sometimes be denoted by LF2-DGTD- $\mathbb{P}_{p} \mathbb{Q}_{k}$ in the following.

- A fourth-order leap-frog scheme (LF4):

$$
\begin{aligned}
& \text { Case (A) : }\left\{\begin{aligned}
\overline{\mathbf{H}}_{i}^{n+\frac{1}{2}}=\overline{\mathbf{H}}_{i}^{n-\frac{1}{2}} & +\frac{\Delta t}{2}\left[\mathcal{X}_{\mu, i}\right]^{-1} \mathbf{A}_{\mathbf{E}^{n}, i} \\
& +\frac{\Delta t^{3}}{24}\left(\overline{S_{h}^{e l} \circ S_{h}^{\text {mag }} \circ S_{h}^{e l}\left(\mathbf{E}^{n}\right)}\right)_{i}, \\
\overline{\mathbf{E}}_{i}^{n+1}=\overline{\mathbf{E}}_{i}^{n} & +\frac{\Delta t}{2}\left[\mathcal{X}_{\epsilon, i}\right]^{-1} \mathbf{A}_{\mathbf{H}^{n+\frac{1}{2}, i}} \\
& +\frac{\Delta t^{3}}{24}\left(\overline{S_{h}^{\text {mag }} \circ S_{h}^{e l} \circ S_{h}^{\text {mag }}\left(\mathbf{H}^{n+\frac{1}{2}}\right)}\right)_{i} .
\end{aligned}\right. \\
& \text { Case (B) : }\left\{\begin{aligned}
\widetilde{\mathbf{H}}_{i}^{n+\frac{1}{2}}=\widetilde{\mathbf{H}}_{i}^{n-\frac{1}{2}} & +\frac{\Delta t}{2}\left[\mathcal{W}_{\mu, i}\right]^{-1} \mathbf{B}_{\mathbf{E}^{n}, i} \\
& +\frac{\Delta t^{3}}{24}\left(S_{h}^{e l} \circ \widetilde{\left.S_{h}^{\text {mag }} \circ S_{h}^{e l}\left(\mathbf{E}^{n}\right)\right)_{i},}\right. \\
\widetilde{\mathbf{E}}_{i}^{n+1}=\widetilde{\mathbf{E}}_{i}^{n}+ & \frac{\Delta t}{2}\left[\mathcal{W}_{\epsilon, i}\right]^{-1} \mathbf{B}_{\mathbf{H}, i}^{n+\frac{1}{2}} \\
& +\frac{\Delta t^{3}}{24}\left(S_{h}^{\text {mag }} \circ \widetilde{S_{h}^{e l} \circ S_{h}^{\text {mag }}}\left(\mathbf{H}^{n+\frac{1}{2}}\right)\right)_{i} .
\end{aligned}\right.
\end{aligned}
$$

The resulting method will sometimes be denoted by LF4-DGTD-P $\mathbb{P}_{p} \mathbb{Q}_{k}$ in the following.

For each of the two schemes, one defines the iteration operator $\mathcal{I} t_{l}^{k}$, with $l=2$ or 4 referring respectively to LF2 or LF4 time schemes, and $k=e l$ or mag:

$$
\mathcal{I} t_{l}^{k}: V_{h} \longrightarrow V_{h},
$$

defined by its degress of freedom for each cases:

- For $l=2$,

$$
\text { Case (A) : }\left\{\begin{array}{l}
\overline{\mathcal{I} t_{2}^{e l}(\mathbf{E})_{i}}=\mathbf{A}_{\mathbf{E}, i}, \\
\overline{\mathcal{I} t_{2}^{m a g}(\mathbf{H})_{i}}=\mathbf{A}_{\mathbf{H}, i} .
\end{array}\right.
$$




$$
\text { Case (B) : }\left\{\begin{array}{l}
\widetilde{\mathcal{I} t_{2}^{\widetilde{m a g}}(\mathbf{H})_{i}}=\mathbf{B}_{\mathbf{E}, i}, \\
\widetilde{\mathcal{I} t_{2}^{e l}(\mathbf{E})_{i}}=\mathbf{B}_{\mathbf{H}, i} .
\end{array}\right.
$$

- For $l=4$,

$$
\begin{gathered}
\text { Case (A) : }\left\{\begin{array}{l}
\overline{\mathcal{I} t_{4}^{e l}(\mathbf{E})_{i}}=\mathbf{A}_{\mathbf{E}, i}+\frac{\Delta t^{2}}{12}\left[\mathcal{X}_{\mu, i}\right]\left(\overline{S_{h}^{e l} \circ S_{h}^{\text {mag }} \circ S_{h}^{e l}(\mathbf{E})}\right)_{i}, \\
\overline{\mathcal{I} t_{4}^{\text {mag }}(\mathbf{H})_{i}}=\mathbf{A}_{\mathbf{H}, i}+\frac{\Delta t^{2}}{12}\left[\mathcal{X}_{\epsilon, i}\right]\left(\overline{S_{h}^{m a g} \circ S_{h}^{e l} \circ S_{h}^{\text {mag }}(\mathbf{E})}\right)_{i} .
\end{array}\right. \\
\text { Case (B) : }\left\{\begin{array}{l}
\widetilde{\mathcal{I} t_{4}^{\text {mag }}(\mathbf{H})_{i}}=\mathbf{B}_{\mathbf{E}, i}+\frac{\Delta t^{2}}{12}\left[\mathcal{W}_{\mu, i}\right]\left(S_{h}^{e l} \circ \widetilde{S_{h}^{\text {mag }} \circ} S_{h}^{e l}(\mathbf{E})\right)_{i}, \\
\widetilde{\mathcal{I} t_{4}^{e l}(\mathbf{E})_{i}}=\mathbf{B}_{\mathbf{H}, i}+\frac{\Delta t^{2}}{12}\left[\mathcal{W}_{\epsilon, i}\right]\left(S_{h}^{\text {mag }} \circ \widetilde{S_{h}^{e l} \circ S_{h}^{m a g}}(\mathbf{E})\right)_{i} .
\end{array}\right.
\end{gathered}
$$

\section{Theoretical aspects}

In this section, for simplicity, we assume that $\Gamma_{a}=\varnothing$. The study for the LF2 scheme will be extensively detailed. Since the scheme of proof for LF4 scheme follows the one from LF2, we will only point out the difficulties added to ensure readability. We begin by studying the $L^{2}$ stability analysis of the proposed DGTD- $\mathbb{P}_{p} \mathbb{Q}_{k}$ method and then conduct an a priori convergence analysis.

\subsection{Stability analysis}

\subsubsection{Preliminaries}

We assume the following hypotheses:

$$
\begin{aligned}
& \forall \mathbf{X} \in\left(\mathbb{P}_{p}\left[c_{i}\right]\right)^{3}, \quad\|\operatorname{rot}(\mathbf{X})\|_{c_{i}} \leq \frac{\alpha_{i}^{\tau} p_{i}}{\left|c_{i}\right|}\|\mathbf{X}\|_{c_{i}}, \\
& \forall \mathbf{X} \in\left(\mathbb{P}_{p}\left[c_{i}\right]\right)^{3}, \quad\|\mathbf{X}\|_{a_{i j}}^{2} \leq \frac{\beta_{i j}^{\tau}\left\|\mathbf{n}_{i j}\right\|}{\left|c_{i}\right|}\|\mathbf{X}\|_{c_{i}}^{2},
\end{aligned}
$$

where $\alpha_{i}^{\tau}$ and $\beta_{i j}^{\tau}\left(\forall j \in \mathcal{V}_{i}\right)$ are constant parameters which do not depend on the discretization parameter $h$, but on the geometry of the finite element (here, a tetrahedron) and on the shape of the basis functions (i.e. on the interpolation degree). We also admit similar hypotheses $\forall \mathbf{X} \in$ $\left(\mathbb{Q}_{k}\left[c_{i}\right]\right)^{3}$ with $\alpha_{i}^{q}$ and $\beta_{i j}^{q}$ defining the constant parameters which are specific to a hexahedron. We will use $\alpha_{i}$ for either $\alpha_{i}^{\tau}$ or $\alpha_{i}^{q}$ if respectively $c_{i}$ is a tetrahedron or a hexahedron. Same notation will hold for $\beta_{i j}$. Besides, $\|\cdot\|_{c_{i}}$ and $\|\cdot\|_{a_{i j}}$ are $L^{2}$ norms, $\mathbf{n}_{i j}$ is the non-unitary normal vector to $a_{i j}$ directed from $c_{i}$ to $c_{j}$ with $\left\|\mathbf{n}_{i j}\right\|=\int_{a_{i j}} 1 d \sigma$ (i.e. the area of the interface $a_{i j}$ ), $\left|c_{i}\right|=\int_{c_{i}} 1 d \mathbf{x}$ (i.e. the volume of $c_{i}$ ). In what follows, we also make use of $p_{i}=\sum_{j \in \mathcal{V}_{i}}\left\|\mathbf{n}_{i j}\right\|$.

Properties of operators $S_{h}^{e l}, S_{h}^{\text {mag }}$.

$\mathrm{RR} \mathrm{n}^{\circ} 8257$ 
Proposition 4.1. In a global formulation, if:

$$
\tilde{\nu}_{i}:=\sum_{j \in \mathcal{V}_{i}}\left(\frac{2}{\sqrt{\mu_{i} \epsilon_{i}}} \frac{\alpha_{i}}{\left|c_{i}\right|}+\frac{1}{\sqrt{\mu_{i} \epsilon_{j}}} \frac{\beta_{i j}}{\left|c_{i}\right|}\right)\left\|\mathbf{n}_{i j}\right\|, \forall i \in[1, N],
$$

and:

$$
\hat{\nu}_{i}:=\sum_{j \in \mathcal{V}_{i}}\left(\frac{2}{\sqrt{\mu_{i} \epsilon_{i}}} \frac{\alpha_{i}}{\left|c_{i}\right|}+\frac{1}{\sqrt{\mu_{j} \epsilon_{i}}} \frac{\beta_{i j}}{\left|c_{i}\right|}\right)\left\|\mathbf{n}_{i j}\right\|, \forall i \in[1, N]
$$

then

$$
\left\|S_{h}^{e l}(\mathbf{E})\right\|_{\mu} \leq 2 \sqrt{\max _{i \in[1, N]}\left(\tilde{\nu}_{i}\right)} \sqrt{\max _{i \in[1, N]}\left(\hat{\nu}_{i}\right)}\|\mathbf{E}\|_{\epsilon}
$$

and:

$$
\left\|S_{h}^{\operatorname{mag}}(\mathbf{H})\right\|_{\epsilon} \leq 2 \sqrt{\max _{i \in[1, N]}\left(\tilde{\nu}_{i}\right)} \sqrt{\max _{i \in[1, N]}\left(\hat{\nu}_{i}\right)}\|\mathbf{H}\|_{\mu}
$$

where $\|v\|_{L_{\epsilon}^{2}\left(c_{i}\right)}=\left(\int_{c_{i}} \epsilon|v|^{2} d x\right)^{\frac{1}{2}}$ and $\|v\|_{L_{\mu}^{2}\left(c_{i}\right)}=\left(\int_{c_{i}} \mu|v|^{2} d x\right)^{\frac{1}{2}}$, for any $v \in L^{2}\left(c_{i}\right), c_{i} \in \mathcal{C}_{h}$, and:

$$
\|v\|_{\mu}^{2}=\sum_{i \in[1, N]}\|v\|_{L_{\mu}^{2}\left(c_{i}\right)}^{2}
$$

and:

$$
\|v\|_{\epsilon}^{2}=\sum_{i \in[1, N]}\|v\|_{L_{\epsilon}^{2}\left(c_{i}\right)}^{2}
$$

Proof. In a matricial form $\left\|S_{h}^{e l}(\mathbf{E})\right\|_{L_{\mu}^{2}\left(c_{i}\right)}^{2}=\left({\overline{S_{h}^{e l}(\mathbf{E})}}_{i}^{\mathrm{T}} \mathcal{X}_{\mu, i}{\overline{S_{h}^{e l}(\mathbf{E})}}_{i}\right.$ if $c_{i}$ is a tetrahedron. One has an analoguous expression if $c_{i}$ is a hexahedron. The exact expression is given by:

$$
\begin{aligned}
\left(\overline{S_{h}^{e l}(\mathbf{E})_{i}}\right)^{\mathrm{T}} \mathcal{X}_{\mu, i} \overline{S_{h}^{e l}(\mathbf{E})_{i}}= & \frac{1}{2}\left(\overline{S_{h}^{e l}(\mathbf{E})_{i}}\right)^{\mathrm{T}} \\
& \left(\sum_{k=1}^{3} \mathcal{X}_{i}^{x_{k}} \overline{\mathbf{E}}_{i}+\sum_{a_{i j} \in \mathscr{T}_{d}^{i}} \mathcal{X}_{i j} \overline{\mathbf{E}}_{j}-\sum_{a_{i j} \in \mathscr{T}_{m}^{i}} \mathcal{X}_{i m} \overline{\mathbf{E}}_{i}+\sum_{a_{i j} \in \mathscr{H}_{d}^{i}} \mathcal{A}_{i j} \widetilde{\mathbf{E}}_{j}\right) .
\end{aligned}
$$

One has:

$$
\sum_{k=1}^{3}\left({\overline{S_{h}^{e l}(\mathbf{E})_{i}}}^{\mathrm{T}} \mathcal{X}_{i}^{x_{k}} \overline{\mathbf{E}}_{i}=-\int_{c_{i}}\left[\left(\operatorname{rot}\left(S_{h}^{e l}(\mathbf{E})_{i}\right)\right) \mathbf{E}_{i}+\left(\operatorname{rot} \mathbf{E}_{i}\right) S_{h}^{e l}(\mathbf{E})_{i}\right] d \mathbf{x}\right.
$$

from which one deduces:

$$
\begin{aligned}
\left|\sum_{k=1}^{3}\left(\overline{S_{h}^{e l}(\mathbf{E})_{i}}\right)^{\mathrm{T}} \mathcal{X}_{i}^{x_{k}} \overline{\mathbf{E}}_{i}\right| & \leq \frac{1}{\sqrt{\epsilon_{i} \mu_{i}}}\left\|\operatorname{rot}\left(S_{h}^{e l}(\mathbf{E})_{i}\right)\right\|_{\mu_{i}, c_{i}}\left\|\mathbf{E}_{i}\right\|_{\epsilon_{i}, c_{i}} \\
& +\left\|\operatorname{rot}\left(\mathbf{E}_{i}\right)\right\|_{\mu_{i}, c_{i}}\left\|S_{h}^{e l}(\mathbf{E})_{i}\right\|_{\epsilon_{i}, c_{i}} d \mathbf{x} .
\end{aligned}
$$

Using the inverse inequalities 22 , one can write:

$$
\|\operatorname{rot}(\mathbf{X})\|_{c_{i}} \leq \frac{\alpha_{i} p_{i}}{\left|c_{i}\right|}\|\mathbf{X}\|_{c_{i}},
$$


so that:

$$
\left|\sum_{k=1}^{3}\left(\overline{S_{h}^{e l}(\mathbf{E})_{i}}\right)^{\mathrm{T}} \mathcal{X}_{i}^{x_{k}} \overline{\mathbf{E}}_{i}\right| \leq \frac{2}{\sqrt{\epsilon_{i} \mu_{i}}} \frac{\alpha_{i}^{\tau} p_{i}}{\left|c_{i}\right|}\left\|S_{h}^{e l}(\mathbf{E})_{i}\right\|_{\mu_{i}, c_{i}}\left\|\mathbf{E}_{i}\right\|_{\epsilon_{i}, c_{i}} .
$$

Furthermore one also has:

$$
\sum_{a_{i j} \in \mathscr{T}_{d}^{i}}\left(\overline{S_{h}^{e l}(\mathbf{E})_{i}}\right)^{\mathrm{T}} \mathcal{X}_{i j} \overline{\mathbf{E}}_{j}=\sum_{a_{i j} \in \mathscr{T}_{d}^{i}} \int_{a_{i j}}\left(S_{h}^{e l}(\mathbf{E})_{i} \times \mathbf{E}_{j}\right) \breve{\mathbf{n}}_{i j} d \sigma
$$

so that:

$$
\left|\sum_{a_{i j} \in \mathscr{T}_{d}^{i}}\left({\overline{S_{h}^{e l}(\mathbf{E})}}_{i}\right)^{\mathrm{T}} \mathcal{X}_{i j} \overline{\mathbf{E}}_{j}\right| \leq \frac{1}{\sqrt{\mu_{i} \epsilon_{j}}} \sum_{a_{i j} \in \mathscr{T}_{d}^{i}}\left\|\sqrt{\mu_{i}} S_{h}^{e l}(\mathbf{E})_{i}\right\|_{a_{i, j}}\left\|\sqrt{\epsilon_{j}} \mathbf{E}_{j}\right\|_{a_{i, j}} .
$$

Using the inverse inequality:

$$
\|\mathbf{X}\|_{a_{i j}}^{2} \leq \frac{\beta_{i j}\left\|\mathbf{n}_{i j}\right\|}{\left|c_{i}\right|}\|\mathbf{X}\|_{c_{i}}^{2}
$$

where $\beta_{i j}$ stands for $\beta_{i j}^{\tau}$ or $\beta_{i j}^{q}$ if respectively $c_{i}$ is a tetrahedron or a hexahedron, one has:

$$
\left|\sum_{a_{i j} \in \mathscr{T}_{d}^{i}}\left(\overline{S_{h}^{e l}(\mathbf{E})_{i}}\right)^{\mathrm{T}} \mathcal{X}_{i j} \overline{\mathbf{E}}_{j}\right| \leq \frac{1}{\sqrt{\mu_{i} \epsilon_{j}}} \sum_{a_{i j} \in \mathscr{T}_{d}^{i}} \sqrt{\frac{\beta_{i j}\left\|\mathbf{n}_{i j}\right\|}{\left|c_{i}\right|}}\left\|S_{h}^{e l}(\mathbf{E})_{i}\right\|_{\mu_{i}, c_{i}} \sqrt{\frac{\beta_{j i}\left\|\mathbf{n}_{j i}\right\|}{\left|c_{j}\right|}}\left\|\mathbf{E}_{j}\right\|_{\epsilon_{j}, c_{j}} .
$$

In a similar way:

$$
\left|\sum_{a_{i j} \in \mathscr{T}_{m}^{i}}\left(\overline{S_{h}^{e l}(\mathbf{E})_{i}}\right)^{\mathrm{T}} \mathcal{X}_{i j} \overline{\mathbf{E}}_{j}\right| \leq \frac{1}{\sqrt{\mu_{i} \epsilon_{j}}} \sum_{a_{i j} \in \mathscr{T}_{m}^{i}} \sqrt{\frac{\beta_{i j}\left\|\mathbf{n}_{i j}\right\|}{\left|c_{i}\right|}}\left\|S_{h}^{e l}(\mathbf{E})_{i}\right\|_{\mu_{i}, c_{i}} \sqrt{\frac{\beta_{j i}\left\|\mathbf{n}_{j i}\right\|}{\left|c_{j}\right|}}\left\|\mathbf{E}_{j}\right\|_{\epsilon_{j}, c_{j}},
$$

with the conventions adopted on the boundaries. The last term writes as:

$$
\left(\overline{S_{h}^{e l}(\mathbf{E})_{i}}\right)^{\mathrm{T}} \sum_{a_{i j} \in \mathscr{H}_{d}^{i}} \mathcal{A}_{i j} \widetilde{\mathbf{E}}_{j}
$$

Since:

$$
{\overline{S_{h}^{e l}(\mathbf{E})_{i}^{\mathrm{T}}}}_{\mathcal{A}_{i j}} \widetilde{\mathbf{E}}_{j}=\int_{a_{i j}}\left(S_{h}^{e l}(\mathbf{E})_{i}^{\mathrm{T}} \times \mathbf{E}_{j}\right)_{\mathbf{n}_{i j}} d \sigma,
$$

one thus also has:

$$
\left.\mid \overline{\left(S_{h}^{e l}(\mathbf{E})_{i}\right.}\right)^{\mathrm{T}} \sum_{a_{i j} \in \mathscr{H}_{d}^{i}} \mathcal{A}_{i j} \widetilde{\mathbf{E}}_{j} \mid \leq \frac{1}{\sqrt{\mu_{i} \epsilon_{j}}} \sum_{a_{i j} \in \mathscr{H}_{d}^{i}} \sqrt{\frac{\beta_{i j}\left\|\mathbf{n}_{i j}\right\|}{\left|c_{i}\right|}}\left\|S_{h}^{e l}(\mathbf{E})_{i}\right\|_{\mu_{i}, c_{i}} \sqrt{\frac{\beta_{j i}\left\|\mathbf{n}_{j i}\right\|}{\left|c_{j}\right|}}\left\|\mathbf{E}_{j}\right\|_{\epsilon_{j}, c_{j}} .
$$

$\operatorname{RR} n^{\circ} 8257$ 
Combining all the terms (26)-(33):

$$
\begin{aligned}
& 2\left(\overline{S_{h}^{e l}(\mathbf{E})_{i}}\right)^{\mathrm{T}} \mathcal{X}_{\mu, i} \overline{S_{h}^{e l}(\mathbf{E})_{i}} \leq \\
& \frac{2}{\sqrt{\mu_{i} \epsilon_{i}}} \frac{\alpha_{i} p_{i}}{\left|c_{i}\right|}\left\|S_{h}^{e l}(\mathbf{E})_{i}\right\|_{\mu_{i}, c_{i}}\left\|\mathbf{E}_{i}\right\|_{\epsilon_{i}, c_{i}}+ \\
& \frac{1}{\sqrt{\mu_{i} \epsilon_{j}}} \sum_{a_{i j} \in \mathscr{T}_{d}^{i}} \sqrt{\frac{\beta_{i j}\left\|\mathbf{n}_{i j}\right\|}{\left|c_{i}\right|}}\left\|S_{h}^{e l}(\mathbf{E})_{i}\right\|_{\mu_{i}, c_{i}} \sqrt{\frac{\beta_{j i}\left\|\mathbf{n}_{j i}\right\|}{\left|c_{j}\right|}}\left\|\mathbf{E}_{j}\right\|_{\epsilon_{j}, c_{j}}+ \\
& \frac{1}{\sqrt{\mu_{i} \epsilon_{j}}} \sum_{a_{i j} \in \mathscr{T}_{m}^{i}} \sqrt{\frac{\beta_{i j}\left\|\mathbf{n}_{i j}\right\|}{\left|c_{i}\right|}}\left\|S_{h}^{e l}(\mathbf{E})_{i}\right\|_{\mu_{i}, c_{i}} \sqrt{\frac{\beta_{j i}\left\|\mathbf{n}_{j i}\right\|}{\left|c_{j}\right|}}\left\|\mathbf{E}_{j}\right\|_{\epsilon_{j}, c_{j}}+ \\
& \frac{1}{\sqrt{\mu_{i} \epsilon_{j}}} \sum_{a_{i j} \in \mathscr{H}_{d}^{i}} \sqrt{\frac{\beta_{i j}\left\|\mathbf{n}_{i j}\right\|}{\left|c_{i}\right|}}\left\|S_{h}^{e l}(\mathbf{E})_{i}\right\|_{\mu_{i}, c_{i}} \sqrt{\frac{\beta_{j i}\left\|\mathbf{n}_{j i}\right\|}{\left|c_{j}\right|}}\left\|\mathbf{E}_{j}\right\|_{\epsilon_{j}, c_{j}} .
\end{aligned}
$$

Let $\gamma_{i j}:=\sqrt{\frac{\beta_{i j}\left\|\mathbf{n}_{i j}\right\|}{\left|c_{i}\right|}}$, then:

$$
\begin{aligned}
& 2 \sum_{i \in\left[1, N_{\tau}\right]}\left(\overline{S_{h}^{e l}(\mathbf{E})_{i}}\right)^{\mathrm{T}} \mathcal{X}_{\mu, i} \overline{S_{h}^{e l}(\mathbf{E})_{i}} \leq \\
& \sum_{i} \frac{2}{\sqrt{\mu_{i} \epsilon_{i}}} \frac{\alpha_{i} p_{i}}{\left|c_{i}\right|}\left\|S_{h}^{e l}(\mathbf{E})_{i}\right\|\left\|_{\mu_{i}, c_{i}}\right\| \mathbf{E}_{i} \|_{\epsilon_{i}, c_{i}}+ \\
& \sum_{i} \frac{1}{\sqrt{\mu_{i} \epsilon_{j}}} \sum_{a_{i j} \in \mathscr{T}_{d}^{i}} \gamma_{i j}\left\|S_{h}^{e l}(\mathbf{E})_{i}\right\|_{\mu_{i}, c_{i}} \gamma_{j i}\left\|\mathbf{E}_{j}\right\|_{\epsilon_{j}, c_{j}}+ \\
& \sum_{i} \frac{1}{\sqrt{\mu_{i} \epsilon_{j}}} \sum_{a_{i j} \in \mathscr{T}_{m}^{i}} \gamma_{i j}\left\|S_{h}^{e l}(\mathbf{E})_{i}\right\|_{\mu_{i}, c_{i}} \gamma_{j i}\left\|\mathbf{E}_{j}\right\|_{\epsilon_{j}, c_{j}}+ \\
& \sum_{i} \frac{1}{\sqrt{\mu_{i} \epsilon_{j}}} \sum_{a_{i j} \in \mathscr{H}_{m}^{i}} \gamma_{i j}\left\|S_{h}^{e l}(\mathbf{E})_{i}\right\|_{\mu_{i}, c_{i}} \gamma_{j i}\left\|\mathbf{E}_{j}\right\|_{\epsilon_{j}, c_{j}} .
\end{aligned}
$$

Thus:

$$
\begin{aligned}
& 2 \sum_{i \in\left[1, N_{\tau}\right]}\left(\overline{S_{h}^{e l}(\mathbf{E})_{i}}\right)^{\mathrm{T}} \mathcal{X}_{\mu, i} \overline{S_{h}^{e l}(\mathbf{E})_{i}} \leq \\
& \sum_{i \in\left[1, N_{\tau}\right]} \frac{2}{\sqrt{\mu_{i} \epsilon_{i}}} \frac{\alpha_{i} p_{i}}{\left|c_{i}\right|}\left\|S_{h}^{e l}(\mathbf{E})_{i}\right\|_{\mu_{i}, c_{i}}\left\|\mathbf{E}_{i}\right\|_{\epsilon_{i}, c_{i}}+ \\
& \sum_{i \in\left[1, N_{\tau}\right]} \sum_{j \in \mathcal{V}_{i}} \frac{1}{\sqrt{\mu_{i} \epsilon_{j}}} \gamma_{i j}\left\|S_{h}^{e l}(\mathbf{E})_{i}\right\|_{\mu_{i}, c_{i}} \gamma_{j i}\left\|\mathbf{E}_{j}\right\|_{\epsilon_{j}, c_{j}} .
\end{aligned}
$$

Similar expressions can be obtained for the case of degrees of freedom associated to hexahedra. This implies:

$$
\begin{aligned}
& 2 \sum_{i \in\left[1, N_{\tau}\right]}\left(\overline{S_{h}^{e l}(\mathbf{E})_{i}}\right)^{\mathrm{T}} \mathcal{X}_{\mu, i} \overline{S_{h}^{e l}(\mathbf{E})_{i}}+2 \sum_{i \in\left[1, N_{q}\right]}\left(\widetilde{S_{h}^{e l}(\mathbf{E})_{i}}\right)^{\mathrm{T}} \mathcal{W}_{\mu, i} \widetilde{S_{h}^{e l}(\mathbf{E})_{i}} \leq \\
& \sum_{i \in[1, N]} \frac{2}{\sqrt{\mu_{i} \epsilon_{i}}} \frac{\alpha_{i} p_{i}}{\left|c_{i}\right|}\left\|S_{h}^{e l}(\mathbf{E})_{i}\right\|_{\mu_{i}, c_{i}}\left\|\mathbf{E}_{i}\right\|_{\epsilon_{i}, c_{i}}+ \\
& \sum_{i \in[1, N]} \sum_{j \in \mathcal{V}_{i}} \frac{1}{\sqrt{\mu_{i} \epsilon_{j}}} \gamma_{i j}\left\|S_{h}^{e l}(\mathbf{E})_{i}\right\|_{\mu_{i}, c_{i}} \gamma_{j i}\left\|\mathbf{E}_{j}\right\|_{\epsilon_{j}, c_{j}} .
\end{aligned}
$$


This gives:

$$
\begin{aligned}
\left\|S_{h}^{e l}(\mathbf{E})\right\|_{\mu}^{2} & \leq\left(\sum_{i=1}^{N} \frac{1}{\sqrt{\mu_{i} \epsilon_{i}}} \frac{\alpha_{i} p_{i}}{\left|c_{i}\right|}\left\|S_{h}^{e l}(\mathbf{E})_{i}\right\|_{\mu_{i}, c_{i}}^{2}\right)^{\frac{1}{2}}\left(\sum_{i=1}^{N} \frac{1}{\sqrt{\mu_{i} \epsilon_{i}}} \frac{\alpha_{i} p_{i}}{\left|c_{i}\right|}\left\|\mathbf{E}_{i}\right\|_{\epsilon_{i}, c_{i}}^{2}\right)^{\frac{1}{2}} \\
& +\left(\sum_{i=1}^{N} \sum_{j \in \mathcal{V}_{i}} \frac{1}{2 \sqrt{\mu_{i} \epsilon_{j}}} \gamma_{i j}^{2}\left\|S_{h}^{e l}(\mathbf{E})_{i}\right\|_{\mu_{i}, c_{i}}^{2}\right)^{\frac{1}{2}}\left(\sum_{i=1}^{N} \sum_{j \in \mathcal{V}_{i}} \frac{1}{2 \sqrt{\mu_{i} \epsilon_{j}}} \gamma_{j i}^{2}\left\|\mathbf{E}_{j}\right\|_{\epsilon_{j}, c_{j}}^{2}\right)^{\frac{1}{2}} .
\end{aligned}
$$

Let $\tilde{\nu}_{i}=\sum_{j \in \mathcal{V}_{i}}\left(\frac{1}{\sqrt{\mu_{i} \epsilon_{i}}} \frac{\alpha_{i}}{\left|c_{i}\right|}\left\|\mathbf{n}_{i j}\right\|+\frac{1}{2 \sqrt{\mu_{i} \epsilon_{j}}} \gamma_{i j}^{2}\right)$, then:

$$
\begin{aligned}
\left\|S_{h}^{e l}(\mathbf{E})\right\|_{\mu}^{2} \leq\left(\sum_{i=1}^{N} \tilde{\nu}_{i}\left\|S_{h}^{e l}(\mathbf{E})_{i}\right\|_{\mu_{i}, c_{i}}^{2}\right)^{\frac{1}{2}} & {\left[\left(\sum_{i=1}^{N} \frac{1}{\sqrt{\mu_{i} \epsilon_{i}}} \frac{\alpha_{i} p_{i}}{\left|c_{i}\right|}\left\|\mathbf{E}_{i}\right\|_{\epsilon_{i}, c_{i}}^{2}\right)^{\frac{1}{2}}+\right.} \\
& \left.\left(\sum_{i=1}^{N} \sum_{a_{i j} \in \mathscr{T}_{d}^{i}} \frac{1}{2 \sqrt{\mu_{j} \epsilon_{i}}} \gamma_{i j}^{2}\left\|\mathbf{E}_{i}\right\|_{\epsilon_{i}, c_{i}}^{2}\right)^{\frac{1}{2}}\right] .
\end{aligned}
$$

Similarly let, $\hat{\nu}_{i}=\sum_{j \in \mathcal{V}_{i}}\left(\frac{1}{\sqrt{\mu_{i} \epsilon_{i}}} \frac{\alpha_{i}^{\tau}}{\left|c_{i}\right|}\left\|\mathbf{n}_{i j}\right\|+\frac{1}{2 \sqrt{\mu_{j} \epsilon_{i}}} \gamma_{i j}^{2}\right)$, then:

$$
\begin{gathered}
\left\|S_{h}^{e l}(\mathbf{E})\right\|_{\mu}^{2} \leq 2\left(\sum_{i=1}^{N} \tilde{\nu}_{i}\left\|S_{h}^{e l}(\mathbf{E})_{i}\right\|_{\mu_{i}, c_{i}}^{2}\right)^{\frac{1}{2}}\left(\sum_{i=1}^{N} \hat{\nu}_{i}\left\|\mathbf{E}_{i}\right\|_{\epsilon_{i}, c_{i}}^{2}\right)^{\frac{1}{2}}, \\
\left\|S_{h}^{e l}(\mathbf{E})\right\|_{\mu}^{2} \leq 2 \max _{i \in[1, N]}\left(\tilde{\nu}_{i}\right) \max _{i}\left(\hat{\nu}_{i \in[1, N]}\right)\left\|S_{h}^{e l}(\mathbf{E})\right\|_{\mu}\|\mathbf{E}\|_{\epsilon} .
\end{gathered}
$$

The result of the proposition then follows.

\section{Proposition 4.2 .}

$$
\ll \mathbf{E}, S_{h}^{m a g}(\mathbf{H}) \gg_{\epsilon}=-\ll \mathbf{H}, S_{h}^{e l}(\mathbf{E}) \gg_{\mu},
$$

where $\ll \cdot, \cdot \gg_{\mu}$ is the $L^{2}$-vectorial scalar product with weight $\mu$ and with an analoguous definition for $\ll \cdot, \cdot \gg_{\epsilon}$.

Proof. One has:

$$
\ll \mathbf{E}, S_{h}^{m a g}(\mathbf{H}) \gg_{\epsilon}=\sum_{c_{i} \in \mathscr{T}_{h}} \ll \mathbf{E}, S_{h}^{m a g}(\mathbf{H}) \gg_{\epsilon_{i}, c_{i}}+\sum_{c_{i} \in \mathscr{Q}_{h}} \ll \mathbf{E}, S_{h}^{m a g}(\mathbf{H}) \gg_{\epsilon_{i}, c_{i}} .
$$

Let us recall the expression of $S_{h}^{e l}, S_{h}^{\text {mag }}$ :

$$
\begin{aligned}
& \overline{\left(S_{h}^{e l}(\mathbf{E})\right)_{i}}=\frac{1}{2} \mathcal{X}_{\mu, i}^{-1}\left(\sum_{k=1}^{3} \mathcal{X}_{i}^{x_{k}} \overline{\mathbf{E}}_{i}+\sum_{a_{i j} \in \mathscr{T}_{d}^{i}} \mathcal{X}_{i j} \overline{\mathbf{E}}_{j}-\sum_{a_{i j} \in \mathscr{T}_{m}^{i}} \mathcal{X}_{i m} \overline{\mathbf{E}}_{i}+\sum_{a_{i j} \in \mathscr{H}_{d}^{i}} \mathcal{A}_{i j} \widetilde{\mathbf{E}}_{j}\right), \\
& \widetilde{\left(S_{h}^{e l}(\mathbf{E})\right)_{i}}=\frac{1}{2} \mathcal{W}_{\mu, i}^{-1}\left(\sum_{k=1}^{3} \mathcal{W}_{i}^{x_{k}} \widetilde{\mathbf{E}}_{i}+\sum_{a_{i j} \in \mathscr{Q}_{d}^{i}} \mathcal{W}_{i j} \widetilde{\mathbf{E}}_{j}-\sum_{a_{i j} \in \mathscr{Q}_{m}^{i}} \mathcal{W}_{i m} \widetilde{\mathbf{E}}_{i}+\sum_{a_{i j} \in \mathscr{H}_{d}^{i}} \mathcal{B}_{i j} \overline{\mathbf{E}}_{j}\right),
\end{aligned}
$$

$\mathrm{RR} \mathrm{n}^{\circ} 8257$ 


$$
\begin{aligned}
{\overline{\left(S_{h}^{\text {mag }}(\mathbf{H})\right)_{i}}}_{i} & =-\frac{1}{2} \mathcal{X}_{\epsilon, i}^{-1}\left(\sum_{k=1}^{3} \mathcal{X}_{i}^{x_{k}} \overline{\mathbf{H}}_{i}+\sum_{a_{i j} \in \mathscr{T}_{d}^{i}} \mathcal{X}_{i j} \overline{\mathbf{H}}_{j}+\sum_{a_{i j} \in \mathscr{T}_{m}^{i}} \mathcal{X}_{i m} \overline{\mathbf{H}}_{i}+\sum_{a_{i j} \in \mathscr{H}_{d}^{i}} \mathcal{A}_{i j} \widetilde{\mathbf{H}}_{j}\right) \\
\left(\widetilde{\left.S_{h}^{\text {mag }}(\mathbf{H})\right)_{i}}\right. & =-\frac{1}{2} \mathcal{W}_{\epsilon, i}^{-1}\left(\sum_{k=1}^{3} \mathcal{W}_{i}^{x_{k}} \widetilde{\mathbf{H}}_{i}+\sum_{a_{i j} \in \mathscr{Q}_{d}^{i}} \mathcal{W}_{i j} \widetilde{\mathbf{H}}_{j}+\sum_{a_{i j} \in \mathscr{Q}_{m}^{i}} \mathcal{W}_{i m} \widetilde{\mathbf{H}}_{i}+\sum_{a_{i j} \in \mathscr{H}_{d}^{i}} \mathcal{B}_{i j} \overline{\mathbf{H}}_{j}\right) .
\end{aligned}
$$

Thus one has to consider terms like:

$$
\begin{array}{r}
\sum_{c_{i} \in \mathscr{T}_{h}} \ll \mathbf{E}, S_{h}^{\text {mag }}(\mathbf{H}) \gg_{\epsilon_{i}, c_{i}}=-\frac{1}{2} \sum_{c_{i} \in \mathscr{T}_{h}} \overline{\mathbf{E}}_{i}^{\mathrm{T}}\left(\sum_{k=1}^{3} \mathcal{X}_{i}^{x_{k}} \overline{\mathbf{H}}_{i}+\sum_{a_{i j} \in \mathscr{T}_{d}^{i}} \mathcal{X}_{i j} \overline{\mathbf{H}}_{j}+\right. \\
\left.\sum_{a_{i j} \in \mathscr{T}_{m}^{i}} \mathcal{X}_{i m} \overline{\mathbf{H}}_{i}+\sum_{a_{i j} \in \mathscr{H}_{d}^{i}} \mathcal{A}_{i j} \widetilde{\mathbf{H}}_{j}\right) .
\end{array}
$$

Since $\mathcal{X}_{i}^{x_{k}}$ is symmetric:

$$
\sum_{c_{i} \in \mathscr{T}_{h}} \overline{\mathbf{E}}_{i}^{\mathrm{T}} \sum_{k=1}^{3} \mathcal{X}_{i}^{x_{k}} \overline{\mathbf{H}}_{i}=\sum_{c_{i} \in \mathscr{T}_{h}} \overline{\mathbf{H}}_{i}^{\mathrm{T}} \sum_{k=1}^{3} \mathcal{X}_{i}^{x_{k}} \overline{\mathbf{E}}_{i},
$$

with analoguous expressions for hybrid faces. Furthermore, due to the symmetry (or antisymmetry) of the operator involved in the representative matrices:

$$
\begin{aligned}
& \sum_{c_{i} \in \mathscr{T}_{h}} \overline{\mathbf{E}}_{i}^{\mathrm{T}} \sum_{a_{i j} \in \mathscr{T}_{d}^{i}} \mathcal{X}_{i j} \overline{\mathbf{H}}_{j}=\sum_{c_{j} \in \mathscr{T}_{h}} \overline{\mathbf{H}}_{j}^{\mathrm{T}} \sum_{a_{j i} \in \mathscr{T}_{d}^{j}} \mathcal{X}_{j i} \overline{\mathbf{E}}_{i} . \\
& \sum_{c_{i} \in \mathscr{T}_{h}} \overline{\mathbf{E}}_{i}^{\mathrm{T}} \sum_{a_{i j} \in \mathscr{T}_{m}^{i}} \mathcal{X}_{i m} \overline{\mathbf{H}}_{i}=-\sum_{c_{i} \in \mathscr{T}_{h}} \overline{\mathbf{H}}_{i}^{\mathrm{T}} \sum_{a_{i j} \in \mathscr{T}_{m}^{i}} \mathcal{X}_{i m} \overline{\mathbf{E}}_{i},
\end{aligned}
$$

with analoguous expressions for hybrid faces. Moreover:

$$
\begin{aligned}
\sum_{c_{i} \in \mathscr{T}_{h}} & \overline{\mathbf{E}}_{i}^{\mathrm{T}} \sum_{a_{i j} \in \mathscr{H}_{d}^{i}} \mathcal{A}_{i j} \widetilde{\mathbf{H}}_{j}+\sum_{c_{i} \in \mathscr{Q}_{h}} \widetilde{\mathbf{E}}_{i}^{\mathrm{T}} \sum_{a_{i j} \in \mathscr{H}_{d}^{i}} \mathcal{B}_{i j} \overline{\mathbf{H}}_{j}= \\
& \sum_{c_{i} \in \mathscr{T}_{h}} \overline{\mathbf{H}}_{i}^{\mathrm{T}} \sum_{a_{i j} \in \mathscr{H}_{d}^{i}} \mathcal{A}_{i j} \widetilde{\mathbf{E}}_{j}+\sum_{c_{i} \in \mathscr{Q}_{h}} \widetilde{\mathbf{H}}_{i}^{\mathrm{T}} \sum_{a_{i j} \in \mathscr{H}_{d}^{i}} \mathcal{B}_{i j} \overline{\mathbf{E}}_{j} .
\end{aligned}
$$

The result of the proposition then follows.

Discrete energy. We define the following discrete electromagnetic energy:

$$
\mathfrak{E}^{n}=\frac{1}{2}\left[\sum_{i=1}^{N_{\tau}} \mathfrak{A}^{n}+\sum_{i=1}^{N_{q}} \mathfrak{B}^{n}\right],
$$

with:

$$
\begin{aligned}
& \mathfrak{A}^{n}=\left(\overline{\mathbf{E}}_{i}^{n}\right)^{\mathrm{T}} \mathcal{X}_{\epsilon, i} \overline{\mathbf{E}}_{i}^{n}+\left(\overline{\mathbf{H}}_{i}^{n-\frac{1}{2}}\right)^{\mathrm{T}} \mathcal{X}_{\mu, i} \overline{\mathbf{H}}_{i}^{n+\frac{1}{2}}, \\
& \mathfrak{B}^{n}=\left(\widetilde{\mathbf{E}}_{i}^{n}\right)^{\mathrm{T}} \mathcal{W}_{\epsilon, i} \widetilde{\mathbf{E}}_{i}^{n}+\left(\widetilde{\mathbf{H}}_{i}^{n-\frac{1}{2}}\right)^{\mathrm{T}} \mathcal{W}_{\mu, i} \widetilde{\mathbf{H}}_{i}^{n+\frac{1}{2}},
\end{aligned}
$$


and where $N_{\tau}$ and $N_{q}$ respectively denote the numbers of tetrahedra and hexahedra in $\mathscr{T}_{h}$ and $\mathscr{Q}_{h}$. For proving the stability of the proposed DGTD- $\mathbb{P}_{p} \mathbb{Q}_{k}$ method, we first verify that $\mathfrak{E}^{n}$ is conserved through one time step, i.e. $\Delta \mathfrak{E}=\mathfrak{E}^{n+1}-\mathfrak{E}^{n}=0$, and then find the condition on $\Delta t$ under which $\mathfrak{E}^{n}$ is a positive definite quadratic form. This yields a CFL-like sufficient stability condition.

\subsubsection{Conservation of the discrete energy}

The variation of the discrete energy through one time step is given by:

$$
\Delta \mathfrak{E}=\frac{1}{2}\left[\sum_{i=1}^{N_{\tau}}\left(\mathfrak{A}^{n+1}-\mathfrak{A}^{n}\right)+\sum_{i=1}^{N_{q}}\left(\mathfrak{B}^{n+1}-\mathfrak{B}^{n}\right)\right] .
$$

We introduce the following quantities that will be used in the sequel:

$$
\Delta \mathbf{E}^{n+1}=\mathbf{E}^{n+1}-\mathbf{E}^{n}, \quad \nabla \mathbf{E}^{n+1}=\mathbf{E}^{n+1}+\mathbf{E}^{n},
$$

And in a same manner, one can define the vector of degrees of freedom on the element $c_{i}$. Similary:

$$
\begin{aligned}
& \Delta \overline{\mathbf{E}}_{i}^{n+1}=\overline{\mathbf{E}}_{i}^{n+1}-\overline{\mathbf{E}}_{i}^{n}, \Delta \widetilde{\mathbf{E}}_{i}^{n+1}=\widetilde{\mathbf{E}}_{i}^{n+1}-\widetilde{\mathbf{E}}_{i}^{n}, \\
& \nabla \overline{\mathbf{E}}_{i}^{n+1}=\overline{\mathbf{E}}_{i}^{n+1}+\overline{\mathbf{E}}_{i}^{n}, \quad \nabla \widetilde{\mathbf{E}}_{i}^{n+1}=\widetilde{\mathbf{E}}_{i}^{n+1}+\widetilde{\mathbf{E}}_{i}^{n},
\end{aligned}
$$

Using the symmetry of $\mathcal{X}_{\epsilon, i}$ and $\mathcal{X}_{\mu, i}$ we have that:

$$
\begin{aligned}
\left(\overline{\mathbf{E}}_{i}^{n+1}\right)^{\mathrm{T}} \mathcal{X}_{\epsilon, i} \overline{\mathbf{E}}_{i}^{n+1} & -\left(\overline{\mathbf{E}}_{i}^{n}\right)^{\mathrm{T}} \mathcal{X}_{\epsilon, i} \overline{\mathbf{E}}_{i}^{n}=\left(\nabla \overline{\mathbf{E}}_{i}^{n+1}\right)^{\mathrm{T}} \mathcal{X}_{\epsilon, i} \Delta \overline{\mathbf{E}}_{i}^{n+1}, \\
\left(\overline{\mathbf{H}}_{i}^{n+\frac{1}{2}}\right)^{\mathrm{T}} \mathcal{X}_{\mu, i} \overline{\mathbf{H}}_{i}^{n+\frac{3}{2}} & -\left(\overline{\mathbf{H}}_{i}^{n-\frac{1}{2}}\right)^{\mathrm{T}} \mathcal{X}_{\mu, i} \overline{\mathbf{H}}_{i}^{n+\frac{1}{2}} \\
& =\left(\overline{\mathbf{H}}_{i}^{n+\frac{1}{2}}\right)^{\mathrm{T}} \mathcal{X}_{\mu, i}\left(\overline{\mathbf{H}}_{i}^{n+\frac{3}{2}}-\overline{\mathbf{H}}_{i}^{n+\frac{1}{2}}+\overline{\mathbf{H}}_{i}^{n+\frac{1}{2}}-\overline{\mathbf{H}}_{i}^{n-\frac{1}{2}}\right),
\end{aligned}
$$

and similarly for the terms appearing in the summation over the hexahedral elements. Then:

$$
\begin{aligned}
\Delta \mathfrak{E}=\frac{1}{2}\left[\sum_{i=1}^{N_{\tau}}(\right. & \left(\nabla \overline{\mathbf{E}}_{i}^{n+1}\right)^{\mathrm{T}} \mathcal{X}_{\epsilon, i}\left(\Delta \overline{\mathbf{E}}_{i}^{n+1}\right)+ \\
& \left.\left(\overline{\mathbf{H}}_{i}^{n+\frac{1}{2}}\right)^{\mathrm{T}} \mathcal{X}_{\mu, i}\left(\overline{\mathbf{H}}_{i}^{n+\frac{3}{2}}-\overline{\mathbf{H}}_{i}^{n+\frac{1}{2}}+\overline{\mathbf{H}}_{i}^{n+\frac{1}{2}}-\overline{\mathbf{H}}_{i}^{n-\frac{1}{2}}\right)\right)+ \\
\sum_{i=1}^{N_{q}}( & \left(\nabla \widetilde{\mathbf{E}}_{i}^{n+1}\right)^{\mathrm{T}} \mathcal{W}_{\epsilon, i}\left(\Delta \widetilde{\mathbf{E}}_{i}^{n+1}\right)+ \\
& \left.\left.\left(\widetilde{\mathbf{H}}_{i}^{n+\frac{1}{2}}\right)^{\mathrm{T}} \mathcal{W}_{\mu, i}\left(\widetilde{\mathbf{H}}_{i}^{n+\frac{3}{2}}-\widetilde{\mathbf{H}}_{i}^{n+\frac{1}{2}}+\widetilde{\mathbf{H}}_{i}^{n+\frac{1}{2}}-\widetilde{\mathbf{H}}_{i}^{n-\frac{1}{2}}\right)\right)\right],
\end{aligned}
$$

and:

$$
\begin{aligned}
& \Delta \mathfrak{E}=\frac{\Delta t}{4}\left[\sum_{i=1}^{N_{\tau}}\left(\left(\nabla \overline{\mathbf{E}}_{i}^{n+1}\right)^{\mathrm{T}} \overline{\mathcal{I} t_{l}^{m a g}\left(\mathbf{H}^{n+\frac{1}{2}}\right)_{i}}+\left(\overline{\mathbf{H}}_{i}^{n+\frac{1}{2}}\right)^{\mathrm{T}}\left(\overline{\mathcal{I} t_{l}^{e l}\left(\mathbf{E}^{n+1}\right)_{i}}+\overline{\mathcal{I} t_{l}^{e l}\left(\mathbf{E}^{n}\right)_{i}}\right)\right)+\right. \\
& \text { RR n }{ }^{\circ} 8257 \\
& \left.\left.\sum_{i=1}^{N_{q}}\left(\left(\nabla \widetilde{\mathbf{E}}_{i}^{n+1}\right)^{\mathrm{T}} \widetilde{\mathcal{I} t_{l}^{m a g}\left(\mathbf{H}^{n+\frac{1}{2}}\right)_{i}}+\left(\widetilde{\mathbf{H}}_{i}^{n+\frac{1}{2}}\right)^{\mathrm{T}}\left(\widetilde{\mathcal{I} t_{l}^{l\left(\mathbf{E}^{n+1}\right.}}\right)_{i}+\widetilde{\mathcal{I} t_{l}^{e l}\left(\mathbf{E}^{n}\right)_{i}}\right)\right)\right] .
\end{aligned}
$$


Due to the expressions of the iteration operator if $l=2$, one has to treat terms like:

$$
\Delta \mathfrak{E}=\frac{\Delta t}{2}\left[\ll \nabla \mathbf{E}^{n+1}, S_{h}^{m a g}\left(\mathbf{H}^{n+\frac{1}{2}}\right) \gg_{\epsilon}+\ll \mathbf{H}^{n+\frac{1}{2}}, S_{h}^{e l}\left(\mathbf{E}^{n+1}\right)+S_{h}^{e l}\left(\mathbf{E}^{n}\right) \gg_{\mu}\right] .
$$

Now, one can apply Proposition 4.2 and deduce directly that for $l=2$ (i.e. LF2 case):

$$
\Delta \mathfrak{E}=\frac{\Delta t}{2}\left[\ll \nabla \mathbf{E}^{n+1}, S_{h}^{m a g}\left(\mathbf{H}^{n+\frac{1}{2}}\right) \gg_{\epsilon}-\ll \nabla \mathbf{E}^{n+1}, S_{h}^{m a g}\left(\mathbf{H}^{n+\frac{1}{2}}\right) \gg_{\epsilon}\right],
$$

so that:

$$
\Delta \mathfrak{E}=0 .
$$

In the case of the LF4 scheme, one furthermore has:

$$
\begin{aligned}
\Delta \mathfrak{E}=\frac{\Delta t}{2}[ & \left.\ll \nabla \mathbf{E}^{n+1}, S_{h}^{m a g}\left(\mathbf{H}^{n+\frac{1}{2}}\right) \gg_{\epsilon}+\ll \mathbf{H}^{n+\frac{1}{2}}, S_{h}^{e l}\left(\mathbf{E}^{n+1}\right)+S_{h}^{e l}\left(\mathbf{E}^{n}\right) \gg_{\mu}\right] \\
+\frac{\Delta t^{3}}{48}[ & \ll \nabla \mathbf{E}^{n+1}, S_{h}^{\text {mag }} \circ S_{h}^{e l} \circ S_{h}^{m a g}\left(\mathbf{H}^{n+\frac{1}{2}}\right) \gg_{\epsilon}+ \\
& \left.\ll \mathbf{H}^{n+\frac{1}{2}}, S_{h}^{e l} \circ S_{h}^{m a g} \circ S_{h}^{e l}\left(\mathbf{E}^{n+1}+\mathbf{E}^{n}\right) \gg_{\mu}\right] .
\end{aligned}
$$

Applying three times Proposition 4.2 to higher order terms in $\Delta t$ involved in this expression, one finds:

$$
\begin{aligned}
\Delta \mathfrak{E}=\frac{\Delta t}{2}[ & \left.\ll \nabla \mathbf{E}^{n+1}, S_{h}^{\text {mag }}\left(\mathbf{H}^{n+\frac{1}{2}}\right) \gg_{\epsilon}-\ll \nabla \mathbf{E}^{n+1}, S_{h}^{\text {mag }}\left(\mathbf{H}^{n+\frac{1}{2}}\right) \gg_{\epsilon}\right] \\
+\frac{\Delta t^{3}}{48}[ & \ll \nabla \mathbf{E}^{n+1}, S_{h}^{\text {mag }} \circ S_{h}^{e l} \circ S_{h}^{\text {mag }}\left(\mathbf{H}^{n+\frac{1}{2}}\right) \gg_{\epsilon}- \\
& \left.\ll \nabla \mathbf{E}^{n+1}, S_{h}^{\text {mag }} \circ S_{h}^{e l} \circ S_{h}^{\text {mag }}\left(\mathbf{H}^{n+\frac{1}{2}}\right) \gg_{\epsilon}\right]=0 .
\end{aligned}
$$

\subsubsection{Stability condition}

We now look for the condition under which $\mathfrak{E}^{n}$ is a positive definite quadratic form. We first note that:

$$
\begin{aligned}
\mathfrak{E}^{n}= & \frac{1}{2}\left[\sum_{i=1}^{N_{\tau}}\left(\left(\overline{\mathbf{E}}_{i}^{n}\right)^{\mathrm{T}} \mathcal{X}_{\epsilon, i} \overline{\mathbf{E}}_{i}^{n}+\left(\overline{\mathbf{H}}_{i}^{n-\frac{1}{2}}\right)^{\mathrm{T}} \mathcal{X}_{\mu, i} \overline{\mathbf{H}}_{i}^{n+\frac{1}{2}}\right)+\right. \\
& \left.\sum_{i=1}^{N_{q}}\left(\left(\widetilde{\mathbf{E}}_{i}^{n}\right)^{\mathrm{T}} \mathcal{W}_{\epsilon, i} \widetilde{\mathbf{E}}_{i}^{n}+\left(\widetilde{\mathbf{H}}_{i}^{n-\frac{1}{2}}\right)^{\mathrm{T}} \mathcal{W}_{\mu, i} \widetilde{\mathbf{H}}_{i}^{n+\frac{1}{2}}\right)\right] \\
= & \frac{1}{2}\left[\sum_{i=1}^{N_{\tau}}\left(\left\|\mathbf{E}_{i}^{n}\right\|_{\epsilon_{i}, \tau_{i}}^{2}+\left\|\mathbf{H}_{i}^{n-\frac{1}{2}}\right\|_{\mu_{i}, \tau_{i}}^{2}+\frac{\Delta t}{2}\left(\overline{\mathbf{H}}_{i}^{n-\frac{1}{2}}\right)^{\mathrm{T}} \widetilde{\mathcal{I} t_{l}^{e l}\left(\mathbf{E}^{n}\right)_{i}}\right)+\right. \\
& \left.\sum_{i=1}^{N_{q}}\left(\left\|\mathbf{E}_{i}^{n}\right\|_{\epsilon_{i}, q_{i}}^{2}+\left\|\mathbf{H}_{i}^{n-\frac{1}{2}}\right\|_{\mu_{i}, q_{i}}^{2}+\frac{\Delta t}{2}\left(\widetilde{\mathbf{H}}_{i}^{n-\frac{1}{2}}\right)^{\mathrm{T}} \widetilde{\mathcal{I} t_{l}^{e l}\left(\mathbf{E}^{n}\right)_{i}}\right)\right] .
\end{aligned}
$$

If $l=2$, we consider the LF2 scheme:

$$
\sum_{i=1}^{N_{\tau}} \frac{\Delta t}{2}\left(\overline{\mathbf{H}}_{i}^{n-\frac{1}{2}}\right)^{\mathrm{T}} \overline{\mathcal{I} t_{l}^{e l}\left(\mathbf{E}^{n}\right)_{i}}+\sum_{i=1}^{N_{q}} \frac{\Delta t}{2}\left(\widetilde{\mathbf{H}}_{i}^{n-\frac{1}{2}}\right)^{\mathrm{T}} \widetilde{\mathcal{I} t_{l}^{e l}\left(\mathbf{E}^{n}\right)_{i}}=\Delta t \ll \mathbf{H}^{n-\frac{1}{2}}, S_{h}^{e l}\left(\mathbf{E}^{n}\right) \gg_{\mu} .
$$

Using the properties of $S_{h}$, one deduces:

$$
\ll \mathbf{H}^{n-\frac{1}{2}}, S_{h}^{e l}\left(\mathbf{E}^{n}\right) \gg_{\mu} \geq-\left\|\mathbf{H}^{n-\frac{1}{2}}\right\|_{\mu}\left\|S_{h}^{e l}\left(\mathbf{E}^{n}\right)\right\|_{\mu},
$$


and by proposition 4.1

$$
\ll \mathbf{H}^{n-\frac{1}{2}}, S_{h}^{e l}\left(\mathbf{E}^{n}\right) \gg_{\mu} \geq-\left\|\mathbf{H}^{n-\frac{1}{2}}\right\|_{\mu} 2 \sqrt{\max _{i \in[1, N]}\left(\tilde{\nu}_{i}\right)} \sqrt{\max _{i \in[1, N]}\left(\hat{\nu}_{i}\right)}\left\|\mathbf{E}^{n}\right\|_{\epsilon},
$$

so that:

$$
\ll \mathbf{H}^{n-\frac{1}{2}}, S_{h}^{e l}\left(\mathbf{E}^{n}\right) \gg_{\mu} \geq-\max _{i \in[1, N]}\left(\tilde{\nu}_{i}\right)\left\|\mathbf{H}^{n-\frac{1}{2}}\right\|_{\mu}^{2}-\max _{i \in[1, N]}\left(\hat{\nu}_{i}\right)\left\|\mathbf{E}^{n}\right\|_{\epsilon}^{2} .
$$

Thus, if:

$$
1-\Delta t \max _{i \in[1, N]}\left(\tilde{\nu}_{i}\right)>0
$$

and:

$$
1-\Delta t \max _{i \in[1, N]}\left(\hat{\nu}_{i}\right)>0
$$

the energy is positive.

If $l=4$, then the only difference lies in the expression of the operator $\mathcal{I}_{l}^{e l}$. One then has to consider a term expressed by:

$$
\frac{\Delta t^{3}}{24} \ll \mathbf{H}^{n-\frac{1}{2}}, S_{h}^{e l} \circ S_{h}^{m a g} \circ S_{h}^{e l}\left(\mathbf{E}^{n}\right) \gg_{\mu} .
$$

Using the same inequalities applied to the operators $S_{h}^{e l}$ and $S_{h}^{m a g}$, one deduces that under the conditions:

$$
1-\Delta t \max _{i \in[1, N]}\left(\tilde{\nu}_{i}\right)-\frac{\Delta t^{3}}{6} \max _{i \in[1, N]}\left(\tilde{\nu}_{i}\right)^{3}>0
$$

and:

$$
1-\Delta t \max _{i \in[1, N]}\left(\hat{\nu}_{i}\right)-\frac{\Delta t^{3}}{6} \max _{i \in[1, N]}\left(\hat{\nu}_{i}\right)^{3}>0,
$$

the energy is positive.

\subsection{Convergence analysis}

In this section we are concerned with the convergence analysis of the DGTD-P $\mathbb{P}_{p}$ method and we aim at providing an a priori error estimation, extending what was previously done in 9 (see also [18] for a related study). We first recall that [1] admits a unique solution $\mathbf{W}$ (see [9]) belonging to the following functional space:

$$
\mathbf{W} \in \mathcal{C}^{1}\left(\left[0, t_{f}\right],\left(L^{2}(\Omega)\right)^{6}\right) \bigcap \mathcal{C}^{0}\left(\left[0, t_{f}\right],(H(\operatorname{rot}, \Omega))^{6}\right),
$$

for any initial condition $\mathbf{W}_{0} \in(H(\operatorname{rot}, \Omega))^{6}$ satisfying the boundary conditions introduced in section 2. Moreover, we associate to the weak formulations (7) and (8) (with unknown $\mathbf{W}_{h}(t)$ ) the initial condition $\mathbf{W}_{h}(0)=P_{h}\left(\mathbf{W}_{0}\right)$ with $P_{h}:\left(L^{2}(\Omega)\right)^{6} \rightarrow V_{h}^{6}$ the orthogonal projector on $V_{h}^{6}$ for the scalar product in $\left(L^{2}(\Omega)\right)^{6}$ (by abuse of notation, we will use $P_{h}$ when necessary, for the orthogonal projection on $V_{h}^{3}$ ). We now introduce some assumptions and a few additional notations.

$\mathrm{RR} \mathrm{n}^{\circ} 8257$ 


\subsubsection{Propositions, lemmas, assumptions and notations}

In the following, $h_{c_{i}}$ denotes the diameter of a cell (tetrahedron or hexaedron) $c_{i}$. We consider a family of multi-element non-conforming meshes $\left(\mathscr{C}_{h}\right)_{h}$ where $h$ is the discretization parameter of each mesh defined as $h=\max _{c_{i} \in \mathscr{C}_{h}} h_{c_{i}}$. The meshes $\mathscr{C}_{h}$ are supposed to match to the boundary of the domain $\partial \Omega$, i.e. the discretized volume $\Omega_{h}=\bigcup_{c_{i} \in \mathscr{C}_{h}} c_{i}$ is equal to $\Omega$. We assume that the meshes $\mathscr{C}_{h}$ are uniformly shape regular i.e. that there exists a constant $\eta>0$ such that:

$$
\forall h, \forall c_{i} \in \mathscr{C}_{h}, \quad h_{c_{i}} / \rho_{c_{i}} \leq \eta
$$

where $\rho_{c_{i}}$ is the diameter of the largest sphere included in element $c_{i}$. We also assume (inverse assumption) that there exists a constant $\gamma>0$ (independent of $h$ ) such that:

$$
\forall h, \forall c_{i} \in \mathscr{C}_{h}, \forall j \in \mathcal{V}_{i}, \quad h_{c_{i}} / h_{c_{j}} \leq \gamma .
$$

In addition, the electromagnetic parameters $\epsilon$ and $\mu$ are assumed uniformly bounded and piecewise constants. We denote by $\Omega_{j}$ the subdomains of $\Omega$ with constant values of $\epsilon$ and $\mu$ :

We then introduce the broken Sobolev spaces $P H^{s+1}(\Omega)=\left\{v \mid \forall j, v_{\mid \Omega_{j}} \in H^{s+1}\left(\Omega_{j}\right)\right\}$ equipped with the norm $\|v\|_{P H^{s+1}(\Omega)}=\left(\sum_{j}\left\|v_{\mid \Omega_{j}}\right\|_{s+1, \Omega_{j}}^{2}\right)^{1 / 2}$, where $\|\cdot\|_{s+1, \Omega_{j}}$ stands for the classical $H^{s+1}$ norm on $\Omega_{j}$. Finally, we state three bilinear forms that we will extensively used in the development of the convergence analysis:

$$
\begin{aligned}
m\left(\mathbf{T}, \mathbf{T}^{\prime}\right)= & 2 \int_{\Omega}\left\langle Q \mathbf{T}, \mathbf{T}^{\prime}\right\rangle d \mathbf{x}, \\
a\left(\mathbf{T}, \mathbf{T}^{\prime}\right)= & \int_{\Omega}\left(\left\langle\sum_{k=1}^{3} \partial_{x_{k}}^{h} \mathcal{N}^{k} \mathbf{T}, \mathbf{T}^{\prime}\right\rangle-\sum_{k=1}^{3}\left\langle\partial_{x_{k}}^{h} \mathbf{T}^{\prime}, \mathcal{N}^{k} \mathbf{T}\right\rangle\right) d \mathbf{x}, \\
b\left(\mathbf{T}, \mathbf{T}^{\prime}\right)= & \int_{\mathscr{F}_{d}}\left(\left\langle\{\mathbf{V}\}, \llbracket \mathbf{U}^{\prime} \rrbracket\right\rangle-\left\langle\{\mathbf{U}\}, \llbracket \mathbf{V}^{\prime} \rrbracket\right\rangle-\right. \\
& \left.\left\langle\left\{\mathbf{V}^{\prime}\right\}, \llbracket \mathbf{U} \rrbracket\right\rangle+\left\langle\left\{\mathbf{U}^{\prime}\right\}, \llbracket \mathbf{V} \rrbracket\right\rangle\right) d \sigma \\
+ & \int_{\mathscr{F}_{m}}\left(\left\langle\mathbf{U}, \breve{\mathbf{n}} \times \mathbf{V}^{\prime}\right\rangle+\left\langle\mathbf{V}, \breve{\mathbf{n}} \times \mathbf{U}^{\prime}\right\rangle\right) d \sigma,
\end{aligned}
$$

where:

- $\mathbf{T}=\left(\begin{array}{c}\mathbf{U} \\ \mathbf{V}\end{array}\right) \in \mathbb{R}^{6}$ and $\mathbf{T}^{\prime}=\left(\begin{array}{c}\mathbf{U}^{\prime} \\ \mathbf{V}^{\prime}\end{array}\right) \in \mathbb{R}^{6}\left(\mathbf{U}, \mathbf{V}, \mathbf{U}^{\prime}\right.$ and $\mathbf{V}^{\prime}$ being vectors of $\left.\mathbb{R}^{3}\right)$.

- $\llbracket \mathbf{U}_{h} \rrbracket_{i j}=\left(\left.\mathbf{U}_{j}\right|_{a_{i j}}-\left.\mathbf{U}_{i}\right|_{a_{i j}}\right) \times \breve{\mathbf{n}}_{i j}$ and $\left\{\mathbf{U}_{h}\right\}_{i j}=\frac{\mathbf{U}_{\left.i\right|_{a_{i j}}}+\left.\mathbf{U}_{j}\right|_{a_{i j}}}{2}$ respectively denote the jump and the average of $\mathbf{U}_{h}$ on the face $a_{i j}$.

- $\mathscr{F}_{d}$ is the set of internal faces and $\mathscr{F}_{m}$ the set of metallic faces of the mesh.

- $\left.\left(\partial_{x_{k}}^{h} \mathbf{T}\right)\right|_{c_{i}}=\partial_{x_{k}}\left(\left.\mathbf{T}\right|_{c_{i}}\right)$ pour $k=1,2,3$.

- In the integral over $\mathscr{F}_{m}$, on each face $a_{i j}, \mathbf{U}$ and $\mathbf{V}$ are defined as the restrictions $\left.\mathbf{U}\right|_{c_{i}}$ and $\left.\mathbf{V}\right|_{c_{i}}$. 
Now, computing the sum of (7) over each $c_{i} \in \mathscr{T}_{h}$ and that of (8) over each $c_{i} \in \mathscr{Q}_{h}$, we obtain that the discrete solution $\mathbf{W}_{h} \in V_{h}^{6}$ satisfies:

$$
m\left(\partial_{t} \mathbf{W}_{h}, \mathbf{T}^{\prime}\right)+a\left(\mathbf{W}_{h}, \mathbf{T}^{\prime}\right)+b\left(\mathbf{W}_{h}, \mathbf{T}^{\prime}\right)=0, \quad \forall \mathbf{T}^{\prime} \in V_{h}^{6} .
$$

We then show in Proposition 1 that the DGTD-P $\mathbb{Q}_{k}$ is a consistent approximation of system (1).

Proposition 1. Let $\mathbf{W}$ be the exact solution of (1) belonging to (39). Then:

$$
m\left(\partial_{t} \mathbf{W}, \mathbf{T}^{\prime}\right)+a\left(\mathbf{W}, \mathbf{T}^{\prime}\right)+b\left(\mathbf{W}, \mathbf{T}^{\prime}\right)=0, \quad \forall \mathbf{T}^{\prime} \in V_{h}^{6}
$$

Proof. First, we have that $m\left(\partial_{t} \mathbf{W}, \mathbf{T}^{\prime}\right)$ verifies:

$$
m\left(\partial_{t} \mathbf{W}, \mathbf{T}^{\prime}\right)=2 \sum_{i=1}^{N} \int_{c_{i}}\left\langle Q \partial_{t} \mathbf{W}, \mathbf{T}^{\prime}\right\rangle d \mathbf{x} .
$$

Then, we consider:

$$
\begin{aligned}
b\left(\mathbf{W}, \mathbf{T}^{\prime}\right)= & \int_{\mathscr{F}_{d}}\left(\left\langle\{\mathbf{H}\}, \llbracket \mathbf{U}^{\prime} \rrbracket\right\rangle-\left\langle\{\mathbf{E}\}, \llbracket \mathbf{V}^{\prime} \rrbracket\right\rangle-\right. \\
& \left.\left\langle\left\{\mathbf{V}^{\prime}\right\}, \llbracket \mathbf{E} \rrbracket\right\rangle+\left\langle\left\{\mathbf{U}^{\prime}\right\}, \llbracket \mathbf{H} \rrbracket\right\rangle\right) d \sigma+ \\
& \int_{\mathscr{F}_{m}}\left(\left\langle\mathbf{E}, \breve{\mathbf{n}} \times \mathbf{V}^{\prime}\right\rangle+\left\langle\mathbf{H}, \breve{\mathbf{n}} \times \mathbf{U}^{\prime}\right\rangle\right) d \sigma \\
= & \sum_{a_{i j} \in \mathscr{F}_{d}} \int_{a_{i j}}\left(\left\langle\{\mathbf{H}\}_{i j}, \llbracket \mathbf{U}^{\prime} \rrbracket_{i j}\right\rangle-\left\langle\{\mathbf{E}\}_{i j}, \llbracket \mathbf{V}^{\prime} \rrbracket_{i j}\right\rangle-\right. \\
& \sum_{a_{i j} \in \mathscr{F}_{m}} \int_{a_{i j}}\left(\left\langle\left.\mathbf{E}\right|_{c_{i}}, \breve{\mathbf{n}}_{i j} \times \mathbf{V}_{i}^{\prime}\right\rangle+\left\langle\left.\mathbf{H}\right|_{c_{i}}, \breve{\mathbf{n}}_{i j} \times \mathbf{U}_{i}^{\prime}\right\rangle\right) d \sigma .
\end{aligned}
$$

Since the components of $\mathbf{E}$ and $\mathbf{H}$ belong to $H(\operatorname{rot}, \Omega)$, their tangential traces are continuous. In other words, on $a_{i j} \in \mathscr{F}_{d}$, we have that $\left.\mathbf{E}\right|_{c_{i}} \times \breve{\mathbf{n}}_{i j}=\left.\mathbf{E}\right|_{c_{j}} \times \breve{\mathbf{n}}_{i j}$ and $\left.\mathbf{H}\right|_{c_{i}} \times \breve{\mathbf{n}}_{i j}=\left.\mathbf{H}\right|_{c_{j}} \times \breve{\mathbf{n}}_{i j}$. Thus:

$$
\llbracket \mathbf{E} \rrbracket_{i j}=\llbracket \mathbf{H} \rrbracket_{i j}=0 \quad, \quad\{\mathbf{H}\}_{i j} \times \breve{\mathbf{n}}_{i j}=\mathbf{H} \times \breve{\mathbf{n}}_{i j} \quad \text { and } \quad\{\mathbf{E}\}_{i j} \times \breve{\mathbf{n}}_{i j}=\mathbf{E} \times \breve{\mathbf{n}}_{i j} .
$$

Moreover, still for the integral over $a_{i j} \in \mathscr{F}_{d}$, we can make use of the following relations resulting from the properties of the mixed product:

$$
\begin{aligned}
\left\langle\{\mathbf{H}\}_{i j}, \llbracket \mathbf{U}^{\prime} \rrbracket_{i j}\right\rangle & =\left\langle\{\mathbf{H}\}_{i j} \times \breve{\mathbf{n}}_{i j}, \mathbf{U}_{i}^{\prime}-\mathbf{U}_{j}^{\prime}\right\rangle \\
\left\langle\{\mathbf{E}\}_{i j}, \llbracket \mathbf{V}^{\prime} \rrbracket_{i j}\right\rangle & =\left\langle\{\mathbf{E}\}_{i j} \times \breve{\mathbf{n}}_{i j}, \mathbf{V}_{i}^{\prime}-\mathbf{V}_{j}^{\prime}\right\rangle .
\end{aligned}
$$

Finally, in the integral over $a_{i j} \in \mathscr{F}_{m}$, we have that:

$$
\begin{aligned}
& \left\langle\left.\mathbf{E}\right|_{c_{i}}, \breve{\mathbf{n}}_{i j} \times \mathbf{V}_{i}^{\prime}\right\rangle=\left\langle\left.\mathbf{E}\right|_{c_{i}} \times \breve{\mathbf{n}}_{i j}, \mathbf{V}_{i}^{\prime}\right\rangle=\left\langle\mathbf{E} \times \breve{\mathbf{n}}_{i j}, \mathbf{V}_{i}^{\prime}\right\rangle, \\
& \left\langle\left.\mathbf{H}\right|_{c_{i}}, \breve{\mathbf{n}}_{i j} \times \mathbf{U}_{i}^{\prime}\right\rangle=\left\langle\left.\mathbf{H}\right|_{c_{i}} \times \breve{\mathbf{n}}_{i j}, \mathbf{U}_{i}^{\prime}\right\rangle=\left\langle\mathbf{H} \times \breve{\mathbf{n}}_{i j}, \mathbf{U}_{i}^{\prime}\right\rangle .
\end{aligned}
$$

$\mathrm{RR} \mathrm{n}^{\circ} 8257$ 
We are lead to the following expression for $b\left(\mathbf{W}, \mathbf{T}^{\prime}\right)$ :

$$
\begin{aligned}
b\left(\mathbf{W}, \mathbf{T}^{\prime}\right) & =\sum_{a_{i j} \in \mathscr{F}_{d}} \int_{a_{i j}}\left(\left\langle\mathbf{H} \times \breve{\mathbf{n}}_{i j}, \mathbf{U}_{i}^{\prime}-\mathbf{U}_{j}^{\prime}\right\rangle-\left\langle\mathbf{E} \times \breve{\mathbf{n}}_{i j}, \mathbf{V}_{i}^{\prime}-\mathbf{V}_{j}^{\prime}\right\rangle\right) d \sigma \\
& +\sum_{a_{i j} \in \mathscr{F}_{m}} \int_{a_{i j}}\left(\left\langle\mathbf{E} \times \breve{\mathbf{n}}_{i j}, \mathbf{V}_{i}^{\prime}\right\rangle+\left\langle\mathbf{H} \times \breve{\mathbf{n}}_{i j}, \mathbf{U}_{i}^{\prime}\right\rangle\right) d \sigma .
\end{aligned}
$$

To conclude, we consider the $a\left(\mathbf{W}, \mathbf{T}^{\prime}\right)$ term:

$$
\begin{aligned}
a\left(\mathbf{W}, \mathbf{T}^{\prime}\right) & =\int_{\Omega}\left\langle\sum_{k=1}^{3} \partial_{x_{k}}^{h} \mathcal{N}^{k} \mathbf{W}, \mathbf{T}^{\prime}\right\rangle d \mathbf{x}-\int_{\Omega} \sum_{k=1}^{3}\left\langle\partial_{x_{k}}^{h} \mathbf{T}^{\prime}, \mathcal{N}^{k} \mathbf{W}\right\rangle d \mathbf{x} \\
& =\sum_{i=1}^{N} \int_{c_{i}}\left\langle\sum_{k=1}^{3} \partial_{x_{k}} \mathcal{N}^{k} \mathbf{W}, \mathbf{T}^{\prime}\right\rangle d \mathbf{x} \\
& -\sum_{i=1}^{N} \int_{c_{i}} \sum_{k=1}^{3}\left\langle\partial_{x_{k}} \mathbf{T}^{\prime}, \mathcal{N}^{k} \mathbf{W}\right\rangle d \mathbf{x} .
\end{aligned}
$$

By using the integration by parts formula:

$$
\begin{aligned}
\int_{c_{i}} \sum_{k=1}^{3}\left\langle\partial_{x_{k}} \mathbf{T}^{\prime}, \mathcal{N}^{k} \mathbf{W}\right\rangle d \mathbf{x} & =-\int_{c_{i}}\left\langle\sum_{k=1}^{3} \partial_{x_{k}} \mathcal{N}^{k} \mathbf{W}, \mathbf{T}^{\prime}\right\rangle d \mathbf{x} \\
& +\int_{\partial c_{i}} \sum_{k=1}^{3}\left\langle\mathbf{T}^{\prime}, \breve{\mathbf{n}}^{k}\left(\mathcal{N}^{k} \mathbf{W}\right)\right\rangle d \sigma
\end{aligned}
$$

we obtain, $\mathscr{F}_{d}^{i}$ being the set of the internal faces of the cell $c_{i}$ and $\mathscr{F}_{m}^{i}$ the set of its metallic faces:

$$
\begin{aligned}
a\left(\mathbf{W}, \mathbf{T}^{\prime}\right) & =2 \sum_{i=1}^{N} \int_{c_{i}}\left\langle\sum_{k=1}^{3} \partial_{x_{k}} \mathcal{N}^{k} \mathbf{W}, \mathbf{T}^{\prime}\right\rangle d \mathbf{x}-\sum_{i=1}^{N} \int_{\partial c_{i}} \sum_{k=1}^{3}\left\langle\mathbf{T}^{\prime}, \breve{\mathbf{n}}^{k}\left(\mathcal{N}^{k} \mathbf{W}\right)\right\rangle d \sigma \\
& =2 \sum_{i=1}^{N} \int_{c_{i}}\left\langle\nabla \cdot F(\mathbf{W}), \mathbf{T}^{\prime}\right\rangle d \mathbf{x}-\sum_{i=1}^{N} \sum_{j \in \mathcal{V}_{i}} \int_{a_{i j}} \sum_{k=1}^{3}\left\langle\mathbf{T}^{\prime}, \breve{\mathbf{n}}_{i j}^{k}\left(\mathcal{N}^{k} \mathbf{W}\right)\right\rangle d \sigma \\
& =2 \sum_{i=1}^{N} \int_{c_{i}}\left\langle\nabla \cdot F(\mathbf{W}), \mathbf{T}^{\prime}\right\rangle d \mathbf{x}-\sum_{i=1}^{N} \sum_{j \in \mathcal{V}_{i}} \int_{a_{i j}}\left\langle\sum_{k=1}^{3} \breve{\mathbf{n}}_{i j}^{k} \mathcal{N}^{k} \mathbf{W}, \mathbf{T}^{\prime}\right\rangle d \sigma \\
& =2 \sum_{i=1}^{N} \int_{c_{i}}\left\langle\nabla \cdot F(\mathbf{W}), \mathbf{T}^{\prime}\right\rangle d \mathbf{x}-\sum_{i=1}^{N} \sum_{j \in \mathcal{V}_{i}} \int_{a_{i j}}\left\langle\mathcal{M}_{i j} \mathbf{W}, \mathbf{T}^{\prime}\right\rangle d \sigma \\
& =2 \sum_{i=1}^{N} \int_{c_{i}}\left\langle\nabla \cdot F(\mathbf{W}), \mathbf{T}^{\prime}\right\rangle d \mathbf{x}-\sum_{i=1}^{N} \sum_{a_{i j} \in \mathscr{F}_{d}^{i}} \int_{a_{i j}}\left\langle\mathcal{M}_{i j} \mathbf{W}, \mathbf{T}^{\prime}\right\rangle d \sigma \\
& -\sum_{i=1}^{N} \sum_{a_{i j} \in \mathscr{F}_{m}^{i}} \int_{a_{i j}}\left\langle\mathcal{M}_{i j} \mathbf{W}, \mathbf{T}^{\prime}\right\rangle d \sigma
\end{aligned}
$$


and at last:

$$
\begin{aligned}
a\left(\mathbf{W}, \mathbf{T}^{\prime}\right) & =2 \sum_{i=1}^{N} \int_{c_{i}}\left\langle\nabla \cdot F(\mathbf{W}), \mathbf{T}^{\prime}\right\rangle d \mathbf{x} \\
& -\sum_{i=1}^{N} \sum_{a_{i j} \in \mathscr{F}_{d}^{i}} \int_{a_{i j}}\left(\left\langle\left.\mathbf{H}\right|_{c_{i}} \times \breve{\mathbf{n}}_{i j}, \mathbf{U}_{i}^{\prime}\right\rangle-\left\langle\left.\mathbf{E}\right|_{c_{i}} \times \breve{\mathbf{n}}_{i j}, \mathbf{V}_{i}^{\prime}\right\rangle\right) d \sigma \\
& -\sum_{i=1}^{N} \sum_{a_{i j} \in \mathscr{F}_{m}^{i}} \int_{a_{i j}}\left(\left\langle\left.\mathbf{H}\right|_{c_{i}} \times \breve{\mathbf{n}}_{i j}, \mathbf{U}_{i}^{\prime}\right\rangle-\left\langle\left.\mathbf{E}\right|_{c_{i}} \times \breve{\mathbf{n}}_{i j}, \mathbf{V}_{i}^{\prime}\right\rangle\right) d \sigma
\end{aligned}
$$

We now perform the sum over all the internal and metallic faces. $a\left(\mathbf{W}, \mathbf{T}^{\prime}\right)$ becomes:

$$
\begin{aligned}
a\left(\mathbf{W}, \mathbf{T}^{\prime}\right)= & 2 \sum_{i=1}^{N} \int_{c_{i}}\left\langle\nabla \cdot F(\mathbf{W}), \mathbf{T}^{\prime}\right\rangle d \mathbf{x} \\
& -\sum_{a_{i j} \in \mathscr{F}_{d}} \int_{a_{i j}}\left(\left\langle\left.\mathbf{H}\right|_{c_{i}} \times \breve{\mathbf{n}}_{i j}, \mathbf{U}_{i}^{\prime}\right\rangle-\left\langle\left.\mathbf{E}\right|_{c_{i}} \times \breve{\mathbf{n}}_{i j}, \mathbf{V}_{i}^{\prime}\right\rangle+\right. \\
& \left.\left\langle\left.\mathbf{H}\right|_{c_{j}} \times \breve{\mathbf{n}}_{j i}, \mathbf{U}_{j}^{\prime}\right\rangle-\left\langle\left.\mathbf{E}\right|_{c_{j}} \times \breve{\mathbf{n}}_{j i}, \mathbf{V}_{j}^{\prime}\right\rangle\right) d \sigma \\
& -\sum_{a_{i j} \in \mathscr{F}_{m}} \int_{a_{i j}}\left(\left\langle\left.\mathbf{H}\right|_{c_{i}} \times \breve{\mathbf{n}}_{i j}, \mathbf{U}_{i}^{\prime}\right\rangle-\left\langle\left.\mathbf{E}\right|_{c_{i}} \times \breve{\mathbf{n}}_{i j}, \mathbf{V}_{i}^{\prime}\right\rangle\right) d \sigma \\
= & 2 \sum_{i=1}^{N} \int_{c_{i}}\left\langle\nabla \cdot F(\mathbf{W}), \mathbf{T}^{\prime}\right\rangle d \mathbf{x} \\
& -\sum_{a_{i j} \in \mathscr{F}_{d}} \int_{a_{i j}}\left(\left\langle\mathbf{H} \times \breve{\mathbf{n}}_{i j}, \mathbf{U}_{i}^{\prime}-\mathbf{U}_{j}^{\prime}\right\rangle-\left\langle\mathbf{E} \times \breve{\mathbf{n}}_{i j}, \mathbf{V}_{i}^{\prime}-\mathbf{V}_{j}^{\prime}\right\rangle\right) d \sigma \\
& -\sum_{a_{i j} \in \mathscr{F}_{m}} \int_{a_{i j}}\left(\left\langle\mathbf{H} \times \breve{\mathbf{n}}_{i j}, \mathbf{U}_{i}^{\prime}\right\rangle-\left\langle\mathbf{E} \times \breve{\mathbf{n}}_{i j}, \mathbf{V}_{i}^{\prime}\right\rangle\right) d \sigma .
\end{aligned}
$$

Gathering the previous expressions of $m\left(\partial_{t} \mathbf{W}, \mathbf{T}^{\prime}\right), a\left(\mathbf{W}, \mathbf{T}^{\prime}\right)$ and $b\left(\mathbf{W}, \mathbf{T}^{\prime}\right)$ we deduce:

$$
\begin{aligned}
m\left(\partial_{t} \mathbf{W}, \mathbf{T}^{\prime}\right)+a\left(\mathbf{W}, \mathbf{T}^{\prime}\right)+b\left(\mathbf{W}, \mathbf{T}^{\prime}\right) & =2 \sum_{i=1}^{N} \int_{c_{i}}\left\langle Q\left(\partial_{t} \mathbf{W}\right), \mathbf{T}^{\prime}\right\rangle d \mathbf{x} \\
& +2 \sum_{i=1}^{N} \int_{c_{i}}\left\langle\nabla \cdot F(\mathbf{W}), \mathbf{T}^{\prime}\right\rangle d \mathbf{x} \\
& +2 \sum_{a_{i j} \in \mathscr{F}_{m}} \int_{a_{i j}}\left\langle\mathbf{E} \times \breve{\mathbf{n}}_{i j}, \mathbf{V}_{i}^{\prime}\right\rangle d \sigma .
\end{aligned}
$$

But, if we choose $\mathbf{T}^{\prime}$ as the vectorial test function in $(3)$. Summing over $c_{i} \in \Omega_{h}$, we obtain:

$$
2 \sum_{i=1}^{N} \int_{c_{i}}\left\langle Q\left(\partial_{t} \mathbf{W}\right), \mathbf{T}^{\prime}\right\rangle d \mathbf{x}+2 \sum_{i=1}^{N} \int_{c_{i}}\left\langle\nabla \cdot F(\mathbf{W}), \mathbf{T}^{\prime}\right\rangle d \mathbf{x}=0 .
$$

By applying the boundary condition on the metallic boundary $\partial \Omega^{m}$, i.e. $\mathbf{E} \times \breve{\mathbf{n}}=0$, we conclude the proof since $m\left(\partial_{t} \mathbf{W}, \mathbf{T}^{\prime}\right)+a\left(\mathbf{W}, \mathbf{T}^{\prime}\right)+b\left(\mathbf{W}, \mathbf{T}^{\prime}\right)=0, \quad \forall \mathbf{T}^{\prime} \in V_{h}^{6}$ for $\mathbf{W}$ the exact solution.

In the sequel we will use the following stability lemma.

$R R n^{\circ} 8257$ 
Lemma 1. For all $\mathbf{T}^{\prime} \in V_{h}^{6}$, we have:

$$
a\left(\mathbf{T}^{\prime}, \mathbf{T}^{\prime}\right)+b\left(\mathbf{T}^{\prime}, \mathbf{T}^{\prime}\right)=0 .
$$

Proof. We note that $a\left(\mathbf{T}^{\prime}, \mathbf{T}^{\prime}\right)$ is such that:

$$
\begin{aligned}
a\left(\mathbf{T}^{\prime}, \mathbf{T}^{\prime}\right) & =\int_{\Omega}\left\langle\sum_{k=1}^{3} \partial_{x_{k}}^{h} \mathcal{N}_{k} \mathbf{T}^{\prime}, \mathbf{T}^{\prime}\right\rangle d \mathbf{x}-\int_{\Omega} \sum_{k=1}^{3}\left\langle\partial_{x_{k}}^{h} \mathbf{T}^{\prime}, \mathcal{N}^{k} \mathbf{T}^{\prime}\right\rangle d \mathbf{x} \\
& =\int_{\Omega} \sum_{k=1}^{3}\left\langle\partial_{x_{k}}^{h} \mathcal{N}^{k} \mathbf{T}^{\prime}, \mathbf{T}^{\prime}\right\rangle d \mathbf{x}-\int_{\Omega} \sum_{k=1}^{3}\left\langle\partial_{x_{k}}^{h} \mathbf{T}^{\prime}, \mathcal{N}^{k} \mathbf{T}^{\prime}\right\rangle d \mathbf{x} \\
& =\int_{\Omega} \sum_{k=1}^{3}\left\langle\mathcal{N}^{k}\left(\partial_{x_{k}}^{h} \mathbf{T}^{\prime}\right), \mathbf{T}^{\prime}\right\rangle d \mathbf{x}-\int_{\Omega} \sum_{k=1}^{3}\left\langle\partial_{x_{k}}^{h} \mathbf{T}^{\prime}, \mathcal{N}^{k} \mathbf{T}^{\prime}\right\rangle d \mathbf{x} \\
& =\int_{\Omega} \sum_{k=1}^{3}\left\langle\partial_{x_{k}}^{h} \mathbf{T}^{\prime},\left(\mathcal{N}^{k}\right)^{\mathrm{T}} \mathbf{T}^{\prime}\right\rangle d \mathbf{x}-\int_{\Omega} \sum_{k=1}^{3}\left\langle\partial_{x_{k}}^{h} \mathbf{T}^{\prime}, \mathcal{N}^{k} \mathbf{T}^{\prime}\right\rangle d \mathbf{x} \\
& =\int_{\Omega} \sum_{k=1}^{3}\left\langle\partial_{x_{k}}^{h} \mathbf{T}^{\prime}, \mathcal{N}^{k} \mathbf{T}^{\prime}\right\rangle d \mathbf{x}-\int_{\Omega} \sum_{k=1}^{3}\left\langle\partial_{x_{k}}^{h} \mathbf{T}^{\prime}, \mathcal{N}^{k} \mathbf{T}^{\prime}\right\rangle d \mathbf{x} \\
& =0 .
\end{aligned}
$$

Similarly, for $b\left(\mathbf{T}^{\prime}, \mathbf{T}^{\prime}\right)$ :

$$
\begin{aligned}
b\left(\mathbf{T}^{\prime}, \mathbf{T}^{\prime}\right)= & \int_{\mathscr{F}_{d}}\left(\left\langle\left\{\mathbf{V}^{\prime}\right\}, \llbracket \mathbf{U}^{\prime} \rrbracket\right\rangle-\left\langle\left\{\mathbf{U}^{\prime}\right\}, \llbracket \mathbf{V}^{\prime} \rrbracket\right\rangle-\right. \\
\left.\left\langle\left\{\mathbf{V}^{\prime}\right\}, \llbracket \mathbf{U}^{\prime} \rrbracket\right\rangle+\left\langle\left\{\mathbf{U}^{\prime}\right\}, \llbracket \mathbf{V}^{\prime} \rrbracket\right\rangle\right) d \sigma+ & \\
& \int_{\mathscr{F}_{m}}\left(\left\langle\mathbf{U}^{\prime}, \breve{\mathbf{n}} \times \mathbf{V}^{\prime}\right\rangle+\left\langle\mathbf{V}^{\prime}, \breve{\mathbf{n}} \times \mathbf{U}^{\prime}\right\rangle\right) d \sigma \\
= & \int_{\mathscr{F}_{m}}\left(\left\langle\mathbf{U}^{\prime}, \breve{\mathbf{n}} \times \mathbf{V}^{\prime}\right\rangle-\left\langle\mathbf{U}^{\prime}, \breve{\mathbf{n}} \times \mathbf{V}^{\prime}\right\rangle\right) d \sigma=0 .
\end{aligned}
$$

In summary:

$$
a\left(\mathbf{T}^{\prime}, \mathbf{T}^{\prime}\right)+b\left(\mathbf{T}^{\prime}, \mathbf{T}^{\prime}\right)=0, \quad \forall \mathbf{T}^{\prime} \in V_{h}^{6}
$$

Remark 4. One can also show that $a$ and $b$ are skew-symmetric forms.

This implies, replacing $\mathbf{T}^{\prime}$ in 42 by the discrete solution $\mathbf{W}_{h} \in V_{h}^{6}$ :

$$
m\left(\partial_{t} \mathbf{W}_{h}, \mathbf{W}_{h}\right)=0
$$

We define the semi-discrete energy $\mathfrak{E}_{h}(t)=\frac{1}{2} m\left(\mathbf{W}_{h}, \mathbf{W}_{h}\right)$. In the present case the semi-discrete energy is constant since $\frac{d}{d t} \mathfrak{E}_{h}(t)=m\left(\partial_{t} \mathbf{W}_{h}, \mathbf{W}_{h}\right)=0$.

We conclude this subsection by recalling classical finite element results. In the following inequalities $|\cdot|_{r, c_{i}}$ denotes the standar semi-norm $H^{r}$ on $c_{i}$ and $\|\cdot\|_{0, \partial c_{i}}$ the standard $L^{2}$ norm on $\partial c_{i}$. 
Lemma 2. (see for instance [2]). Let $\tau_{i} \in \mathscr{T}_{h}$ (respectively $q_{i} \in \mathscr{Q}_{h}$ ) and $u \in H^{s+1}\left(\tau_{i}\right)$ (respectively $u \in H^{s+1}\left(q_{i}\right)$ ) for $s \geq 0$. Let $\Pi$ be a linear operator from $H^{s+1}\left(\tau_{i}\right)$ (respectively $H^{s+1}\left(q_{i}\right)$ ) into $\mathbb{P}_{p}\left[\tau_{i}\right]$ (respectively $\mathbb{Q}_{k}\left[q_{i}\right]$ ) such that $\Pi(u)=u, \forall u \in \mathbb{P}_{p}\left[\tau_{i}\right]$ (respectively $\mathbb{Q}_{k}\left[q_{i}\right]$ ). Then:

$$
\begin{gathered}
|\Pi(u)-u|_{r, \tau_{i}} \leq C h_{\tau_{i}}^{\min \{s, p\}+1-r}\|u\|_{s+1, \tau_{i}}, \quad r=0,1 \\
\|\Pi(u)-u\|_{0, \partial \tau_{i}} \leq C h_{\tau_{i}}^{\min \{s, p\}+1 / 2}\|u\|_{s+1, \tau_{i}}
\end{gathered}
$$

and:

$$
\begin{gathered}
|\Pi(u)-u|_{r, q_{i}} \leq C h_{q_{i}}^{\min \{s, k\}+1-r}\|u\|_{s+1, q_{i}}, \quad r=0,1 \\
\|\Pi(u)-u\|_{0, \partial q_{i}} \leq C h_{q_{i}}^{\min \{s, k\}+1 / 2}\|u\|_{s+1, q_{i}}
\end{gathered}
$$

where $C$ is a strictly positive constant that only depends on $p$ (respectively $k$ ), $s$ and the regularity parameter $\eta$ of the mesh.

Lemma 3. (see for instance [2]). For all $v \in \mathbb{P}_{p}\left[\tau_{i}\right]$ (respectively $v \in \mathbb{Q}_{k}\left[q_{i}\right]$ ) one has:

$$
\begin{aligned}
\|v\|_{0, \partial \tau_{i}} & \leq \hat{C} h_{\tau_{i}}^{-1 / 2}\|v\|_{0, \tau_{i}} \\
\|v\|_{1, \tau_{i}} & \leq \hat{C} h_{\tau_{i}}^{-1}\|v\|_{0, \tau_{i}}
\end{aligned}
$$

and:

$$
\begin{aligned}
\|v\|_{0, \partial q_{i}} & \leq \hat{C} h_{q_{i}}^{-1 / 2}\|v\|_{0, q_{i}}, \\
\|v\|_{1, q_{i}} & \leq \hat{C} h_{q_{i}}^{-1}\|v\|_{0, q_{i}},
\end{aligned}
$$

where $\hat{C}$ is a strictly positive constant that only depends on $p$ (respectively $k$ ), $s$ and the regularity parameter $\eta$ of the mesh.

\subsubsection{Convergence of the semi-discrete problem}

Theorem 1. Let $\left(\mathscr{C}_{h}\right)_{h}$ be a family of multi-element non-conforming meshes satisfying (40) and (41). Let $\epsilon$ and $\mu$ be uniformly bounded piecewise constant quantities. $V_{h}$ is given by (4). Let $\mathbf{W}$ be the exact solution of $\sqrt{1})$ belonging to $\sqrt{39})$ and also to $\mathcal{C}^{0}\left(\left[0, t_{f}\right],\left(P H^{s+1}(\Omega)\right)^{6}\right)$ for $s \geq 0$. Let $\mathbf{W}_{h} \in \mathcal{C}^{1}\left(\left[0, t_{f}\right], V_{h}^{6}\right)$ be the semi-discrete solution associated to (7)-(8) (W and $\mathbf{W}_{h}$ also verify the initial conditions of the problem).

Let $\xi_{h}=\max \left\{h_{\tau}^{\min \{s, p\}}, h_{q}^{\min \{s, k\}}\right\}$ with $h_{\tau}=\max _{\tau_{i} \in \mathscr{T}_{h}}\left(h_{\tau_{i}}\right)$ and $h_{q}=\max _{q_{i} \in \mathscr{Q}_{h}}\left(h_{q_{i}}\right)$. Then, there exists a contant $C>0$ independent of $h$ such that:

$$
\max _{t \in\left[0, t_{f}\right]}\left(\left\|P_{h}(\mathbf{W}(t))-\mathbf{W}_{h}(t)\right\|_{0, \Omega}\right) \leq C \xi_{h} t_{f}\|\mathbf{W}\|_{\mathcal{C}^{0}\left(\left[0, t_{f}\right], P H^{s+1}(\Omega)\right)} .
$$

Proof. Let $\mathbf{w}(t)=\mathbf{W}(t)-\mathbf{W}_{h}(t)=\left(\begin{array}{c}\mathbf{E}(t)-\mathbf{E}_{h}(t) \\ \mathbf{H}(t)-\mathbf{H}_{h}(t)\end{array}\right)=\left(\begin{array}{c}\mathbf{e}(t) \\ \mathbf{h}(t)\end{array}\right)$ be the error vector at time $t \in\left[0, t_{f}\right]$ and $\underline{\zeta}$ the minimal value of $\epsilon$ and $\mu$. We also set $\mathfrak{E}_{P_{h}}(t)=\frac{1}{2} m\left(P_{h}(\mathbf{w}(t)), P_{h}(\mathbf{w}(t))\right.$, so that:

$\mathrm{RR} \mathrm{n}^{\circ} 8257$

$$
\begin{aligned}
\mathfrak{E}_{P_{h}}(t) & =\int_{\Omega}\left\langle Q P_{h}(\mathbf{w}(t)), P_{h}(\mathbf{w}(t))\right\rangle d \mathbf{x} \\
& \geq \underline{\zeta} \int_{\Omega}\left\langle P_{h}(\mathbf{w}(t)), P_{h}(\mathbf{w}(t))\right\rangle d \mathbf{x},
\end{aligned}
$$


and:

$$
\begin{aligned}
& \underline{\zeta} \int_{\Omega}\left\langle P_{h}(\mathbf{w}(t)), P_{h}(\mathbf{w}(t))\right\rangle d \mathbf{x} \\
= & \underline{\zeta} \int_{\Omega}\left(\left\langle P_{h}(\mathbf{e}(t)), P_{h}(\mathbf{e}(t))\right\rangle+\left\langle P_{h}(\mathbf{h}(t)), P_{h}(\mathbf{h}(t))\right\rangle\right) d \mathbf{x} \\
= & \underline{\zeta}\left(P_{h}(\mathbf{e}(t))\left\|_{0, \Omega}^{2}+\right\| P_{h}(\mathbf{h}(t)) \|_{0, \Omega}^{2}\right) .
\end{aligned}
$$

Thus:

$$
\mathfrak{E}_{P_{h}}(t) \geq \underline{\zeta}\left(\left\|P_{h}(\mathbf{e}(t))\right\|_{0, \Omega}^{2}+\left\|P_{h}(\mathbf{h}(t))\right\|_{0, \Omega}^{2}\right) .
$$

Taking into account the discretized initial conditions we have that $\mathfrak{E}_{P_{h}}(0)=0$, and for $t \in\left[0, t_{f}\right]$ :

$$
\begin{aligned}
\mathfrak{E}_{P_{h}}(t)=\int_{0}^{t} \frac{d}{d \varsigma} \mathfrak{E}_{P_{h}}(\varsigma) d \varsigma & =\frac{1}{2} \int_{0}^{t} \frac{d}{d \varsigma} m\left(P_{h}(\mathbf{w}(\varsigma)), P_{h}(\mathbf{w}(\varsigma))\right) d \varsigma \\
& =\int_{0}^{t} m\left(\partial_{\varsigma} P_{h}(\mathbf{w}(\varsigma)), P_{h}(\mathbf{w}(\varsigma))\right) d \varsigma .
\end{aligned}
$$

Since $P_{h}(\mathbf{w}(t)) \in V_{h}^{6}$ we also have that:

$$
a\left(P_{h}(\mathbf{w}(t)), P_{h}(\mathbf{w}(t))\right)+b\left(P_{h}(\mathbf{w}(t)), P_{h}(\mathbf{w}(t))\right)=0 .
$$

Thus:

$$
\begin{aligned}
\mathfrak{E}_{P_{h}}(t)=\int_{0}^{t}( & m\left(\partial_{\varsigma} P_{h}(\mathbf{w}(\varsigma)), P_{h}(\mathbf{w}(\varsigma))\right)+ \\
& a\left(P_{h}(\mathbf{w}(\varsigma)), P_{h}(\mathbf{w}(\varsigma))\right)+ \\
& \left.b\left(P_{h}(\mathbf{w}(\varsigma)), P_{h}(\mathbf{w}(\varsigma))\right)\right) d \varsigma .
\end{aligned}
$$

On the other hand, if we substract (42) from 43 and using $\mathbf{T}^{\prime}=P_{h}(\mathbf{w}(t))$, the bilinearity of $m, a$ and $b$ yields:

$$
\begin{aligned}
m\left(\partial_{t} \mathbf{W}(t)-\partial_{t} \mathbf{W}_{h}(t), P_{h}(\mathbf{w}(t))\right) & +a\left(\mathbf{W}(t)-\mathbf{W}_{h}(t), P_{h}(\mathbf{w}(t))\right) \\
& +b\left(\mathbf{W}(t)-\mathbf{W}_{h}(t), P_{h}(\mathbf{w}(t))\right)=0 .
\end{aligned}
$$

We note that in (56) (because $P_{h}$ is a linear operator and $\left.\mathbf{W}_{h} \in V_{h}^{6}\right), P_{h}(\mathbf{w}(t))=P_{h}(\mathbf{W}(t)-$ $\left.\mathbf{W}_{h}(t)\right)=P_{h}(\mathbf{W}(t))-\mathbf{W}_{h}(t)$, then:

$$
\begin{aligned}
\mathfrak{E}_{P_{h}}(t)=\int_{0}^{t}( & \left.m\left(\partial_{\varsigma} P_{h}(\mathbf{W}(\varsigma))-\partial_{\varsigma} \mathbf{W}_{h}(\varsigma)\right), P_{h}(\mathbf{w}(\varsigma))\right)+ \\
& a\left(P_{h}(\mathbf{W}(\varsigma))-\mathbf{W}_{h}(\varsigma), P_{h}(\mathbf{w}(\varsigma))\right)+ \\
& \left.b\left(P_{h}(\mathbf{W}(\varsigma))-\mathbf{W}_{h}(\varsigma), P_{h}(\mathbf{w}(\varsigma))\right)\right) d \varsigma .
\end{aligned}
$$

Integrating $(57)$ over $[0, t]$ and substracting the result from (58) we obtain (using again the bilinearity of $m, a$ and $b$ ):

$$
\begin{aligned}
\mathfrak{E}_{P_{h}}(t)=\int_{0}^{t}( & m\left(\partial_{\varsigma} P_{h}(\mathbf{W}(\varsigma))-\partial_{\varsigma} \mathbf{W}(\varsigma), P_{h}(\mathbf{w}(\varsigma))\right)+ \\
& a\left(P_{h}(\mathbf{W}(\varsigma))-\mathbf{W}(\varsigma), P_{h}(\mathbf{w}(\varsigma))\right)+ \\
& \left.b\left(P_{h}(\mathbf{W}(\varsigma))-\mathbf{W}(\varsigma), P_{h}(\mathbf{w}(\varsigma))\right)\right) d \varsigma .
\end{aligned}
$$


Thus (see also (55)):

$$
\begin{aligned}
& \underline{\zeta}\left(\left\|P_{h}(\mathbf{e}(t))\right\|_{0, \Omega}^{2}+\left\|P_{h}(\mathbf{h}(t))\right\|_{0, \Omega}^{2}\right) \\
& \leq \int_{0}^{t}(\left.m\left(\partial_{\varsigma} P_{h}(\mathbf{W}(\varsigma))-\partial_{\varsigma} \mathbf{W}(\varsigma)\right), P_{h}(\mathbf{w}(\varsigma))\right)+ \\
& a\left(P_{h}(\mathbf{W}(\varsigma))-\mathbf{W}(\varsigma), P_{h}(\mathbf{w}(\varsigma))\right)+ \\
&\left.b\left(P_{h}(\mathbf{W}(\varsigma))-\mathbf{W}(\varsigma), P_{h}(\mathbf{w}(\varsigma))\right)\right) d \varsigma .
\end{aligned}
$$

Moreover, $P_{h}$ being a linear operator independent of time and uniquely defined, we can deduce:

$$
\begin{aligned}
& m\left(\partial_{\varsigma} P_{h}(\mathbf{W}(\varsigma))-\partial_{\varsigma} \mathbf{W}(\varsigma), P_{h}(\mathbf{w}(\varsigma))\right) \\
= & m\left(P_{h}\left(\partial_{\varsigma} \mathbf{W}(\varsigma)\right)-\partial_{\varsigma} \mathbf{W}(\varsigma), P_{h}(\mathbf{w}(\varsigma))\right) \\
= & 2 \int_{\Omega}\left\langle Q\left[P_{h}\left(\partial_{\varsigma} \mathbf{W}(\varsigma)\right)-\partial_{\varsigma} \mathbf{W}(\varsigma)\right], P_{h}(\mathbf{w}(\varsigma))\right\rangle d \mathbf{x} \\
= & 2 \int_{\Omega}\left\langle P_{h}\left(\partial_{\varsigma} \mathbf{W}(\varsigma)\right)-\partial_{\varsigma} \mathbf{W}(\varsigma), Q^{\mathrm{T}} P_{h}(\mathbf{w}(\varsigma))\right\rangle d \mathbf{x} .
\end{aligned}
$$

But, we recall that $P_{h}$ is the orthogonal projector from $\left(L^{2}(\Omega)\right)^{6}$ to the vectorial subspace $V_{h}^{6}$, and because $\partial_{\varsigma} \mathbf{W}(\varsigma) \in\left(L^{2}(\Omega)\right)^{6}, P_{h}\left(\partial_{\varsigma} \mathbf{W}(\varsigma)\right) \in V_{h}^{6}$ and $Q^{\mathrm{T}} P_{h}(\mathbf{w}(\varsigma)) \in V_{h}^{6}(\epsilon$ and $\mu$ are constants on $c_{i}$ ), we have by definition:

$$
m\left(\partial_{\varsigma} P_{h}(\mathbf{W}(\varsigma))-\partial_{\varsigma} \mathbf{W}(\varsigma), P_{h}(\mathbf{W}(\varsigma))\right)=0
$$

then:

$$
\begin{aligned}
& \underline{\zeta}\left(\left\|P_{h}(\mathbf{e}(t))\right\|_{0, \Omega}^{2}+\left\|P_{h}(\mathbf{h}(t))\right\|_{0, \Omega}^{2}\right) \\
\leq & \int_{0}^{t}\left(a\left(P_{h}(\mathbf{W}(\varsigma))-\mathbf{W}(\varsigma), P_{h}(\mathbf{w}(\varsigma))\right)+b\left(P_{h}(\mathbf{W}(\varsigma))-\mathbf{W}(\varsigma), P_{h}(\mathbf{w}(\varsigma))\right)\right) d \varsigma .
\end{aligned}
$$

We now study the two terms in the above integral over $[0, t]$. From now, we omit the temporal variable in order to simplify the notation and we denote by $\tau_{i}$ and $q_{i}$ the tetrahedral and 
hexahedral cells respectively. For what concerns $a\left(P_{h}(\mathbf{W}(\varsigma))-\mathbf{W}(\varsigma), P_{h}(\mathbf{w}(\varsigma))\right)$ we have:

$$
\begin{aligned}
& a\left(P_{h}(\mathbf{W})-\mathbf{W}, P_{h}(\mathbf{w})\right) \\
&= \int_{\Omega}\left(\left\langle\sum_{k=1}^{3} \partial_{x_{k}}^{h} \mathcal{N}^{k}\left[P_{h}(\mathbf{W})-\mathbf{W}\right], P_{h}(\mathbf{w})\right\rangle-\right. \\
&\left.\sum_{k=1}^{3}\left\langle\partial_{x_{k}}^{h} P_{h}(\mathbf{w}), \mathcal{N}^{k}\left[P_{h}(\mathbf{W})-\mathbf{W}\right]\right\rangle\right) d \mathbf{x} \\
&=\int_{\Omega}\left(\left\langle\sum_{k=1}^{3} \partial_{x_{k}}^{h} \mathcal{N}^{k}\left[P_{h}(\mathbf{W})-\mathbf{W}\right], P_{h}(\mathbf{w})\right\rangle-\right. \\
&\left.=\sum_{\Omega=1}^{3}\langle P_{h}(\mathbf{W})-\mathbf{W}, \underbrace{\left(\mathcal{N}^{k}\right)^{T} \partial_{x_{k}}^{h} P_{h}(\mathbf{w})}_{\in V_{h}^{6}}\rangle\right) d \mathbf{x} \\
&=\sum_{i=1}^{N_{\tau}} \int_{\tau_{i}}\left\langle\sum_{k=1}^{3} \partial_{x_{k}}^{h} \mathcal{N}^{k}\left[P_{h}(\mathbf{W})-\mathbf{W}\right], P_{h}(\mathbf{w})\right\rangle d \mathbf{x} \\
&+\sum_{i=1}^{N_{q}} \int_{q_{i}}\left\langle\sum_{k=1}^{3} \partial_{x_{k}} \mathcal{N}^{k}\left[P_{h}(\mathbf{W})-\mathbf{W}\right], P_{h}(\mathbf{w})\right\rangle d \mathbf{x} \\
&\left.=\mathbf{W}], P_{h}(\mathbf{w})\right\rangle d \mathbf{x} .
\end{aligned}
$$

Using the Cauchy-Schwarz inequality we deduce:

$$
\begin{aligned}
& a\left(P_{h}(\mathbf{W})-\mathbf{W}, P_{h}(\mathbf{w})\right) \\
\leq & \left|a\left(P_{h}(\mathbf{W})-\mathbf{W}, P_{h}(\mathbf{w})\right)\right| \\
\leq & \sum_{i=1}^{N_{\tau}}\left\|\sum_{k=1}^{3} \partial_{x_{k}} \mathcal{N}^{k}\left[P_{h}(\mathbf{W})-\mathbf{W}\right]\right\|_{0, \tau_{i}}\left\|P_{h}(\mathbf{w})\right\|_{0, \tau_{i}}+ \\
& \sum_{i=1}^{N_{q}}\left\|\sum_{k=1}^{3} \partial_{x_{k}} \mathcal{N}^{k}\left[P_{h}(\mathbf{W})-\mathbf{W}\right]\right\|_{0, q_{i}}\left\|P_{h}(\mathbf{w})\right\|_{0, q_{i}} .
\end{aligned}
$$

We set $u(\mathbf{x}, t) \equiv P_{h}(\mathbf{W}(\mathbf{x}, t))-\mathbf{W}(\mathbf{x}, t) \in \mathbb{R}^{6}$, then:

$$
\begin{aligned}
\left\|\sum_{k=1}^{3} \partial_{x_{k}} \mathcal{N}^{k} u\right\|_{0, \tau_{i}} & =\left\|\left(\begin{array}{c}
\partial_{x_{3}} u_{5}-\partial_{x_{2}} u_{6} \\
\partial_{x_{1}} u_{6}-\partial_{x_{3}} u_{4} \\
\partial_{x_{2}} u_{4}-\partial_{x_{1}} u_{5} \\
\partial_{x_{2}} u_{3}-\partial_{x_{3}} u_{2} \\
\partial_{x_{3}} u_{1}-\partial_{x_{1}} u_{3} \\
\partial_{x_{1}} u_{2}-\partial_{x_{2}} u_{1}
\end{array}\right)\right\|_{0, \tau_{i}} \\
& \leq\left\|\partial_{x_{3}} u_{5}\right\|_{0, \tau_{i}}+\left\|\partial_{x_{2}} u_{6}\right\|_{0, \tau_{i}}+\left\|\partial_{x_{1}} u_{6}\right\|_{0, \tau_{i}}+\left\|\partial_{x_{3}} u_{4}\right\|_{0, \tau_{i}} \\
& +\left\|\partial_{x_{2}} u_{4}\right\|_{0, \tau_{i}}+\left\|\partial_{x_{1}} u_{5}\right\|_{0, \tau_{i}}+\left\|\partial_{x_{2}} u_{3}\right\|_{0, \tau_{i}}+\left\|\partial_{x_{3}} u_{2}\right\|_{0, \tau_{i}} \\
& +\left\|\partial_{x_{3}} u_{1}\right\|_{0, \tau_{i}}+\left\|\partial_{x_{1}} u_{3}\right\|_{0, \tau_{i}}+\left\|\partial_{x_{1}} u_{2}\right\|_{0, \tau_{i}}+\left\|\partial_{x_{2}} u_{1}\right\|_{0, \tau_{i}} .
\end{aligned}
$$


But, for each $u_{l}(l=1, \ldots, 6)$, we have that $\left\|\partial_{x_{k}} u_{l}\right\|_{0, \tau_{i}} \leq\left\|\nabla u_{l}\right\|_{0, \tau_{i}}$ for $k=1,2,3$. In addition, the definition of the semi-norm $H^{1}\left(\tau_{i}\right)$ being $\left|u_{l}\right|_{1, \tau_{i}}=\left\|\nabla u_{l}\right\|_{0, \tau_{i}}$, we obtain using the inequality (45) (with $r=1$ ) of Lemma 2

$$
\begin{aligned}
\left\|\sum_{k=1}^{3} \partial_{x_{k}} \mathcal{N}^{k} u\right\|_{0, \tau_{i}} & \leq 2 \sum_{l=1}^{6}\left|u_{l}\right|_{1, \tau_{i}}=2 \sum_{l=1}^{6}\left|\left(P_{h}(\mathbf{W})-\mathbf{W}\right)_{l}\right|_{1, \tau_{i}} \\
\leq & 2 \sum_{j=1}^{6} C h_{\tau_{i}}^{\min \{s, p\}}\left\|(\mathbf{W})_{l}\right\|_{s+1, \tau_{i}} \\
& =2 C h_{\tau_{i}}^{\min \{s, p\}}\left(\left\|E_{x}\right\|_{s+1, \tau_{i}}+\left\|E_{y}\right\|_{s+1, \tau_{i}}+\left\|E_{z}\right\|_{s+1, \tau_{i}}+\right. \\
& \left.\left\|H_{x}\right\|_{s+1, \tau_{i}}+\left\|H_{y}\right\|_{s+1, \tau_{i}}+\left\|H_{z}\right\|_{s+1, \tau_{i}}\right) \\
\leq & 2 C h_{\tau_{i}}^{\min \{s, p\}}\left(3\|\mathbf{E}\|_{s+1, \tau_{i}}+3\|\mathbf{H}\|_{s+1, \tau_{i}}\right) \\
\leq & C h_{\tau_{i}}^{\min \{s, p\}}\left(\|\mathbf{E}\|_{s+1, \tau_{i}}+\|\mathbf{H}\|_{s+1, \tau_{i}}\right) .
\end{aligned}
$$

We now apply the inequality $|m+n| \leq \sqrt{2 m^{2}+2 n^{2}}, \forall m, n \in \mathbb{R}$ to $\|\mathbf{E}\|_{s+1, \tau_{i}}$ and $\|\mathbf{H}\|_{s+1, \tau_{i}}$ to obtain:

$$
\begin{aligned}
\left\|\sum_{k=1}^{3} \partial_{x_{k}} \mathcal{N}^{k}\left[P_{h}(\mathbf{W})-\mathbf{W}\right]\right\|_{0, \tau_{i}} & \leq C h_{\tau_{i}}^{\min \{s, p\}}\left(\|\mathbf{E}\|_{s+1, \tau_{i}}+\|\mathbf{H}\|_{s+1, \tau_{i}}\right) \\
& \leq C h_{\tau_{i}}^{\min \{s, p\}}\left(2\|\mathbf{E}\|_{s+1, \tau_{i}}^{2}+2\|\mathbf{H}\|_{s+1, \tau_{i}}^{2}\right)^{\frac{1}{2}} \\
& \leq C h_{\tau_{i}}^{\min \{s, p\}}\left(\|\mathbf{E}\|_{s+1, \tau_{i}}^{2}+\|\mathbf{H}\|_{s+1, \tau_{i}}^{2}\right)^{\frac{1}{2}} .
\end{aligned}
$$

Similarly, we note that (see also (54)):

$$
\left\|P_{h}(\mathbf{w})\right\|_{0, \tau_{i}}=\left(\left\|P_{h}(\mathbf{e})\right\|_{0, \tau_{i}}^{2}+\left\|P_{h}(\mathbf{h})\right\|_{0, \tau_{i}}^{2}\right)^{\frac{1}{2}} .
$$

Then, proceeding in the same way for the hexahedral cell $q_{i}$ (using the inequality 447) of Lemma 2), 60 becomes:

$$
\begin{aligned}
& a\left(P_{h}(\mathbf{W})-\mathbf{W}, P_{h}(\mathbf{w})\right) \\
\leq & \sum_{i=1}^{N_{\tau}} C h_{\tau_{i}}^{\min \{s, p\}}\left(\|\mathbf{E}\|_{s+1, \tau_{i}}^{2}+\|\mathbf{H}\|_{s+1, \tau_{i}}^{2}\right)^{\frac{1}{2}}\left(\left\|P_{h}(\mathbf{e})\right\|_{0, \tau_{i}}^{2}+\left\|P_{h}(\mathbf{h})\right\|_{0, \tau_{i}}^{2}\right)^{\frac{1}{2}} \\
+ & \sum_{i=1}^{N_{q}} C h_{q_{i}}^{\min \{s, k\}}\left(\|\mathbf{E}\|_{s+1, q_{i}}^{2}+\|\mathbf{H}\|_{s+1, q_{i}}^{2}\right)^{\frac{1}{2}}\left(\left\|P_{h}(\mathbf{e})\right\|_{0, q_{i}}^{2}+\left\|P_{h}(\mathbf{h})\right\|_{0, q_{i}}^{2}\right)^{\frac{1}{2}} .
\end{aligned}
$$

Finally, the Cauchy-Schwarz inequality yields:

$$
\begin{gathered}
\sum_{i=1}^{N_{\tau}}\left(\|\mathbf{E}\|_{s+1, \tau_{i}}^{2}+\|\mathbf{H}\|_{s+1, \tau_{i}}^{2}\right)^{\frac{1}{2}}\left(\left\|P_{h}(\mathbf{e})\right\|_{0, \tau_{i}}^{2}+\left\|P_{h}(\mathbf{h})\right\|_{0, \tau_{i}}^{2}\right)^{\frac{1}{2}} \\
\leq\left(\sum_{i=1}^{N_{\tau}}\left(\|\mathbf{E}\|_{s+1, \tau_{i}}^{2}+\|\mathbf{H}\|_{s+1, \tau_{i}}^{2}\right)\right)^{\frac{1}{2}}\left(\sum_{i=1}^{N_{\tau}}\left(\left\|P_{h}(\mathbf{e})\right\|_{0, \tau_{i}}^{2}+\left\|P_{h}(\mathbf{h})\right\|_{0, \tau_{i}}^{2}\right)\right)^{\frac{1}{2}} .
\end{gathered}
$$


By applying the same inequality to the sum over the hexahedral elements and using the definition of $h_{\tau}, h_{q}$ and $\xi_{h}$ (see also Theorem 1 ) we obtain:

$$
\begin{aligned}
& a\left(P_{h}(\mathbf{W})-\mathbf{W}, P_{h}(\mathbf{w})\right) \\
& \leq C h_{\tau}^{\min \{s, p\}}(\left.\|\mathbf{E}\|_{P H^{s+1}\left(\mathscr{T}_{h}\right)}^{2}+\|\mathbf{H}\|_{P H^{s+1}\left(\mathscr{T}_{h}\right)}^{2}\right)^{\frac{1}{2}} \\
&\left(\left\|P_{h}(\mathbf{e})\right\|_{0, \mathscr{T}_{h}}^{2}+\left\|P_{h}(\mathbf{h})\right\|_{0, \mathscr{T}_{h}}^{2}\right)^{\frac{1}{2}}+ \\
&\left.C h_{q}^{\min \{s, k\}}\left(\|\mathbf{E}\|_{P H^{s+1}\left(\mathscr{Q}_{h}\right)}^{2}+\|\mathbf{H}\|_{P H^{s+1}\left(\mathscr{Q}_{h}\right)}^{2}\right)\right)^{\frac{1}{2}}\left(\left\|P_{h}(\mathbf{e})\right\|_{0, \mathscr{Q}_{h}}^{2}+\left\|P_{h}(\mathbf{h})\right\|_{0, \mathscr{Q}_{h}}^{2}\right)^{\frac{1}{2}} \\
& \leq C \xi_{h}\left(\|\mathbf{E}\|_{P H^{s+1}(\Omega)}^{2}+\|\mathbf{H}\|_{P H^{s+1}(\Omega)}^{2}\right)^{\frac{1}{2}}\left(\left\|P_{h}(\mathbf{e})\right\|_{0, \Omega}^{2}+\left\|P_{h}(\mathbf{h})\right\|_{0, \Omega}^{2}\right)^{\frac{1}{2}} .
\end{aligned}
$$

Concerning the term $b\left(P_{h}(\mathbf{W}(\varsigma))-\mathbf{W}(\varsigma), P_{h}(\mathbf{w}(\varsigma))\right.$ in $(59)$ :

$$
\begin{aligned}
& b\left(P_{h}(\mathbf{W})-\mathbf{W}, P_{h}(\mathbf{w})\right) \\
& \leq\left|b\left(P_{h}(\mathbf{W})-\mathbf{W}, P_{h}(\mathbf{w})\right)\right| \\
& \leq \sum_{a_{i j} \in \mathscr{F}_{d}}\left(\left|\int_{a_{i j}}\left\langle\left\{P_{h}(\mathbf{H})-\mathbf{H}\right\}_{i j}, \llbracket P_{h}(\mathbf{e}) \rrbracket_{i j}\right\rangle d \sigma\right|+\right. \\
&\left|\int_{a_{i j}}\left\langle\left\{P_{h}(\mathbf{E})-\mathbf{E}\right\}_{i j}, \llbracket P_{h}(\mathbf{h}) \rrbracket_{i j}\right\rangle d \sigma\right|+ \\
&\left|\int_{a_{i j}}\left\langle\left\{P_{h}(\mathbf{h})\right\}_{i j}, \llbracket P_{h}(\mathbf{E})-\mathbf{E} \rrbracket_{i j}\right\rangle d \sigma\right|+ \\
&\left.\left|\int_{a_{i j}}\left\langle\left\{P_{h}(\mathbf{e})\right\}_{i j}, \llbracket P_{h}(\mathbf{H})-\mathbf{H} \rrbracket_{i j}\right\rangle d \sigma\right|\right)+ \\
& \sum_{a_{i j} \in \mathscr{F}_{m}}\left(\left|\int_{a_{i j}}\left\langle\left[P_{h}(\mathbf{E})-\mathbf{E}\right]_{c_{i}}, \breve{\mathbf{n}}_{i j} \times\left. P_{h}(\mathbf{h})\right|_{c_{i}}\right\rangle d \sigma\right|+\right. \\
&\left.\left|\int_{a_{i j}}\left\langle\left.\left[P_{h}(\mathbf{H})-\mathbf{H}\right]\right|_{c_{i}}, \breve{\mathbf{n}}_{i j} \times\left. P_{h}(\mathbf{e})\right|_{c_{i}}\right\rangle d \sigma\right|\right) .
\end{aligned}
$$

In the sum over the internal faces (i.e. $a_{i j} \in \mathscr{F}_{d}$ ), $a_{i j}$ can be a hybrid face; then, we (arbitrarily) consider the tetrahedron $\tau_{i}$ and the hexahedron $q_{j}$. We again make use of the Cauchy-Schwarz inequality, as well as of inequalities (46) and (48) of Lemma (2), and 49) and (51) of Lemma 
(3):

$$
\begin{aligned}
& \left|\int_{a_{i j}}\left\langle\left\{P_{h}(\mathbf{H})-\mathbf{H}\right\}_{i j}, \llbracket P_{h}(\mathbf{e}) \rrbracket_{i j}\right\rangle d \sigma\right| \\
\leq & \|\left[\frac{\left.\left[P_{h}(\mathbf{H})-\mathbf{H}\right]\right|_{\tau_{i}}+\left.\left[P_{h}(\mathbf{H})-\mathbf{H}\right]\right|_{q_{j}}}{2} \|_{0, a_{i j}}\right. \\
& \left\|\left(\left.P_{h}(\mathbf{e})\right|_{q_{j}}-\left.P_{h}(\mathbf{e})\right|_{\tau_{i}}\right) \times \breve{\mathbf{n}}_{i j}\right\|_{0, a_{i j}} \\
\leq & \left(\left\|\left.\left[P_{h}(\mathbf{H})-\mathbf{H}\right]\right|_{\tau_{i}}\right\|_{0, a_{i j}}+\left\|\left.\left[P_{h}(\mathbf{H})-\mathbf{H}\right]\right|_{q_{j}}\right\|_{0, a_{i j}}\right) \\
& \left(\left\|\left.P_{h}(\mathbf{e})\right|_{\tau_{i}}\right\|_{0, a_{i j}}+\left\|\left.P_{h}(\mathbf{e})\right|_{q_{j}}\right\|_{0, a_{i j}}\right) \\
\leq & \left(C h_{\tau_{i}}^{\min \{s, p\}+\frac{1}{2}}\|\mathbf{H}\|_{s+1, \tau_{i}}+C h_{q_{j}}^{\min \{s, k\}+\frac{1}{2}}\|\mathbf{H}\|_{s+1, q_{j}}\right) \\
& \left(C h_{\tau_{i}}^{-\frac{1}{2}}\left\|P_{h}(\mathbf{e})\right\|_{0, \tau_{i}}+C h_{q_{j}}^{-\frac{1}{2}}\left\|P_{h}(\mathbf{e})\right\|_{0, q_{j}}\right) \\
\leq & C \max \left\{h_{\tau_{i}}^{\min \{s, p\}+\frac{1}{2}}, h_{q_{j}}^{\min \{s, k\}+\frac{1}{2}}\right\} \max \left\{h_{\tau_{i}}^{-\frac{1}{2}}, h_{q_{j}}^{-\frac{1}{2}}\right\} \\
& \left(\|\mathbf{H}\|_{s+1, \tau_{i}}+\|\mathbf{H}\|_{s+1, q_{j}}\right)\left(\left\|P_{h}(\mathbf{e})\right\|_{0, \tau_{i}}+\left\|P_{h}(\mathbf{e})\right\|_{0, q_{j}}\right) .
\end{aligned}
$$

We shall now look for an upper bound for:

$$
\max \left\{h_{\tau_{i}}^{\min \{s, p\}+\frac{1}{2}}, h_{q_{j}}^{\min \{s, k\}+\frac{1}{2}}\right\} \max \left\{h_{\tau_{i}}^{-\frac{1}{2}}, h_{q_{j}}^{-\frac{1}{2}}\right\} .
$$

The exponents of $h_{\tau_{i}}$ and $h_{q_{j}}$ being different, there are four possibilities:

- $\max \left\{h_{\tau_{i}}^{\min \{s, p\}+\frac{1}{2}}, h_{q_{j}}^{\min \{s, k\}+\frac{1}{2}}\right\} \max \left\{h_{\tau_{i}}^{-\frac{1}{2}}, h_{q_{j}}^{-\frac{1}{2}}\right\}$

$$
=h_{\tau_{i}}^{\min \{s, p\}+\frac{1}{2}} h_{\tau_{i}}^{-\frac{1}{2}}=h_{\tau_{i}}^{\min \{s, p\}} \leq h_{\tau}^{\min \{s, p\}} \leq \xi_{h} \text {. }
$$

- $\quad \max \left\{h_{\tau_{i}}^{\min \{s, p\}+\frac{1}{2}}, h_{q_{j}}^{\min \{s, k\}+\frac{1}{2}}\right\} \max \left\{h_{\tau_{i}}^{-\frac{1}{2}}, h_{q_{j}}^{-\frac{1}{2}}\right\}$

$$
=h_{q_{j}}^{\min \{s, k\}+\frac{1}{2}} h_{q_{j}}^{-\frac{1}{2}}=h_{q_{j}}^{\min \{s, k\}} \leq h_{q}^{\min \{s, k\}} \leq \xi_{h} .
$$

- Using 41 with $c_{i}=\tau_{i}$ and $c_{j}=q_{j}$ (together with the regularity parameter $\gamma$ ), we have:

$$
\begin{aligned}
& \max \left\{h_{\tau_{i}}^{\min \{s, p\}+\frac{1}{2}}, h_{q_{j}}^{\min \{s, k\}+\frac{1}{2}}\right\} \max \left\{h_{\tau_{i}}^{-\frac{1}{2}}, h_{q_{j}}^{-\frac{1}{2}}\right\}=h_{\tau_{i}}^{\min \{s, p\}+\frac{1}{2}} h_{q_{j}}^{-\frac{1}{2}} \\
& \leq h_{\tau_{i}}^{\min \{s, p\}+\frac{1}{2}} \sqrt{\gamma} h_{\tau_{i}}^{-\frac{1}{2}} \leq \sqrt{\gamma} h_{\tau_{i}}^{\min \{s, p\}} \leq \sqrt{\gamma} h_{\tau}^{\min \{s, p\}} \leq \sqrt{\gamma} \xi_{h} .
\end{aligned}
$$

- Using 41 with $c_{i}=q_{j}$ and $c_{j}=\tau_{i}$ we have:

$$
\begin{aligned}
& \max \left\{h_{\tau_{i}}^{\min \{s, p\}+\frac{1}{2}}, h_{q_{j}}^{\min \{s, k\}+\frac{1}{2}}\right\} \max \left\{h_{\tau_{i}}^{-\frac{1}{2}}, h_{q_{j}}^{-\frac{1}{2}}\right\}=h_{q_{j}}^{\min \{s, k\}+\frac{1}{2}} h_{\tau_{i}}^{-\frac{1}{2}} \\
\leq & h_{q_{j}}^{\min \{s, k\}+\frac{1}{2}} \sqrt{\gamma} h_{q_{j}}^{-\frac{1}{2}} \leq \sqrt{\gamma} h_{q_{j}}^{\min \{s, k\}} \leq \sqrt{\gamma} h_{q}^{\min \{s, k\}} \leq \sqrt{\gamma} \xi_{h} .
\end{aligned}
$$

Overall we thus obtain:

$$
\max \left\{h_{\tau_{i}}^{\min \{s, p\}+\frac{1}{2}}, h_{q_{j}}^{\min \{s, k\}+\frac{1}{2}}\right\} \max \left\{h_{\tau_{i}}^{-\frac{1}{2}}, h_{q_{j}}^{-\frac{1}{2}}\right\} \leq C \xi_{h} .
$$

$\operatorname{RR} n^{\circ} 8257$ 
Then, if $a_{i j}$ is a hybrid face (we define $\mathscr{H}_{d}$ as the set of hybrid faces of the mesh):

$$
\begin{aligned}
& \left|\int_{a_{i j}}\left\langle\left\{P_{h}(\mathbf{H})-\mathbf{H}\right\}_{i j}, \llbracket P_{h}(\mathbf{e}) \rrbracket_{i j}\right\rangle d \sigma\right| \\
\leq & C \xi_{h}\left(\|\mathbf{H}\|_{s+1, \tau_{i}}+\|\mathbf{H}\|_{s+1, q_{j}}\right)\left(\left\|P_{h}(\mathbf{e})\right\|_{0, \tau_{i}}+\left\|P_{h}(\mathbf{e})\right\|_{0, q_{j}}\right) \\
\leq & C \xi_{h}\left(\|\mathbf{H}\|_{s+1, \tau_{i}}^{2}+\|\mathbf{H}\|_{s+1, q_{j}}^{2}\right)^{\frac{1}{2}}\left(\left\|P_{h}(\mathbf{e})\right\|_{0, \tau_{i}}^{2}+\left\|P_{h}(\mathbf{e})\right\|_{0, q_{j}}^{2}\right)^{\frac{1}{2}} .
\end{aligned}
$$

If $a_{i j}$ is an internal face shared by two tetrahedra $\tau_{i}$ and $\tau_{j}$, and if we denote by $\mathscr{T}_{d}$ the set of internal faces of the mesh shared by two tetrahedra):

$$
\begin{aligned}
& \left|\int_{a_{i j}}\left\langle\left\{P_{h}(\mathbf{H})-\mathbf{H}\right\}_{i j}, \llbracket P_{h}(\mathbf{e}) \rrbracket_{i j}\right\rangle d \sigma\right| \\
& \leq C h_{\tau}^{\min \{s, p\}}\left(\|\mathbf{H}\|_{s+1, \tau_{i}}^{2}+\|\mathbf{H}\|_{s+1, \tau_{j}}^{2}\right)^{\frac{1}{2}}\left(\left\|P_{h}(\mathbf{e})\right\|_{0, \tau_{i}}^{2}+\left\|P_{h}(\mathbf{e})\right\|_{0, \tau_{j}}^{2}\right)^{\frac{1}{2}} \\
& \leq C \xi_{h}\left(\|\mathbf{H}\|_{s+1, \tau_{i}}^{2}+\|\mathbf{H}\|_{s+1, \tau_{j}}^{2}\right)^{\frac{1}{2}}\left(\left\|P_{h}(\mathbf{e})\right\|_{0, \tau_{i}}^{2}+\left\|P_{h}(\mathbf{e})\right\|_{0, \tau_{j}}^{2}\right)^{\frac{1}{2}} .
\end{aligned}
$$

If $a_{i j}$ is an internal face shared by two hexahedra $q_{i}$ and $q_{j}$, and if we denote by $\mathscr{Q}_{d}$ the set of internal faces of the mesh shared by two hexahedra):

$$
\begin{aligned}
& \left|\int_{a_{i j}}\left\langle\left\{P_{h}(\mathbf{H})-\mathbf{H}\right\}_{i j}, \llbracket P_{h}(\mathbf{e}) \rrbracket_{i j}\right\rangle d \sigma\right| \\
\leq & C h_{q}^{\min \{s, k\}}\left(\|\mathbf{H}\|_{s+1, q_{i}}^{2}+\|\mathbf{H}\|_{s+1, q_{j}}^{2}\right)^{\frac{1}{2}}\left(\left\|P_{h}(\mathbf{e})\right\|_{0, q_{i}}^{2}+\left\|P_{h}(\mathbf{e})\right\|_{0, q_{j}}^{2}\right)^{\frac{1}{2}} \\
\leq & C \xi_{h}\left(\|\mathbf{H}\|_{s+1, q_{i}}^{2}+\|\mathbf{H}\|_{s+1, q_{j}}^{2}\right)^{\frac{1}{2}}\left(\left\|P_{h}(\mathbf{e})\right\|_{0, q_{i}}^{2}+\left\|P_{h}(\mathbf{e})\right\|_{0, q_{j}}^{2}\right)^{\frac{1}{2}} .
\end{aligned}
$$

We obtain the same inequalities for the term (see also (61)):

$$
\left|\int_{a_{i j}}\left\langle\left\{P_{h}(\mathbf{e})\right\}_{i j}, \llbracket P_{h}(\mathbf{H})-\mathbf{H} \rrbracket_{i j}\right\rangle d \sigma\right|,
$$

and similar inequalities (which involve norms of $\mathbf{E}$ and $P_{h}(\mathbf{h})$ ) for the terms:

$$
\begin{aligned}
& \left|\int_{a_{i j}}\left\langle\left\{P_{h}(\mathbf{E})-\mathbf{E}\right\}_{i j}, \llbracket P_{h}(\mathbf{h}) \rrbracket_{i j}\right\rangle d \sigma\right|, \\
& \left|\int_{a_{i j}}\left\langle\left\{P_{h}(\mathbf{h})\right\}_{i j}, \llbracket P_{h}(\mathbf{E})-\mathbf{E} \rrbracket_{i j}\right\rangle d \sigma\right| .
\end{aligned}
$$

On the other hand, still in (61), looking at the sum over metallic faces (i.e. $a_{i j} \in \mathscr{F}_{m}$ ), if $a_{i j}$ is a face belonging to a tetrahedron (let $\mathscr{T}_{m}$ be the set of these faces):

$$
\begin{aligned}
& \left|\int_{a_{i j}}\left\langle\left.\left[P_{h}(\mathbf{H})-\mathbf{H}\right]\right|_{\tau_{i}}, \breve{\mathbf{n}}_{i j} \times\left. P_{h}(\mathbf{e})\right|_{\tau_{i}}\right\rangle d \sigma\right| \\
\leq & \left\|\left.\left[P_{h}(\mathbf{H})-\mathbf{H}\right]\right|_{\tau_{i}}\right\|_{0, a_{i j}}\left\|\left.P_{h}(\mathbf{e})\right|_{\tau_{i}}\right\|_{0, a_{i j}} \\
\leq & C h_{\tau_{i}}^{\min \{s, p\}}\|\mathbf{H}\|_{s+1, \tau_{i}}\left\|P_{h}(\mathbf{e})\right\|_{0, \tau_{i}} \leq C \xi_{h}\|\mathbf{H}\|_{s+1, \tau_{i}}\left\|P_{h}(\mathbf{e})\right\|_{0, \tau_{i}} . \quad \text { Inria }
\end{aligned}
$$


We obtain the same inequality for the term:

$$
\left|\int_{a_{i j}}\left\langle\left.\left[P_{h}(\mathbf{E})-\mathbf{E}\right]\right|_{\tau_{i}}, \breve{\mathbf{n}}_{i j} \times\left. P_{h}(\mathbf{h})\right|_{\tau_{i}}\right\rangle d \sigma\right|,
$$

and similar inequalities if $a_{i j} \in \mathscr{Q}_{m}$, with $\mathscr{Q}_{m}$ the set of metallic faces of the mesh belonging to a hexahedron. We thus deduce the following upper bound:

$$
\begin{gathered}
b\left(P_{h}(\mathbf{W})-\mathbf{W}, P_{h}(\mathbf{w})\right) \\
\leq C \xi_{h}\left(\sum _ { a _ { i j } \in \mathscr { T } _ { d } } \left(\left(\|\mathbf{H}\|_{s+1, \tau_{i}}^{2}+\|\mathbf{H}\|_{s+1, \tau_{j}}^{2}\right)^{\frac{1}{2}}\left(\left\|P_{h}(\mathbf{e})\right\|_{0, \tau_{i}}^{2}+\left\|P_{h}(\mathbf{e})\right\|_{0, \tau_{j}}^{2}\right)^{\frac{1}{2}}+\right.\right. \\
\left.\left(\|\mathbf{E}\|_{s+1, \tau_{i}}^{2}+\|\mathbf{E}\|_{s+1, \tau_{j}}^{2}\right)^{\frac{1}{2}}\left(\left\|P_{h}(\mathbf{h})\right\|_{0, \tau_{i}}^{2}+\left\|P_{h}(\mathbf{h})\right\|_{0, \tau_{j}}^{2}\right)^{\frac{1}{2}}\right)+ \\
\sum_{a_{i j} \in \mathscr{Q}_{d}}\left(\left(\|\mathbf{H}\|_{s+1, q_{i}}^{2}+\|\mathbf{H}\|_{s+1, q_{j}}^{2}\right)^{\frac{1}{2}}\left(\left\|P_{h}(\mathbf{e})\right\|_{0, q_{i}}^{2}+\left\|P_{h}(\mathbf{e})\right\|_{0, q_{j}}^{2}\right)^{\frac{1}{2}}+\right. \\
\left.\left(\|\mathbf{E}\|_{s+1, q_{i}}^{2}+\|\mathbf{E}\|_{s+1, q_{j}}^{2}\right)^{\frac{1}{2}}\left(\left\|P_{h}(\mathbf{h})\right\|_{0, q_{i}}^{2}+\left\|P_{h}(\mathbf{h})\right\|_{0, q_{j}}^{2}\right)^{\frac{1}{2}}\right)+ \\
\sum_{a_{i j} \in \mathscr{H}_{d}}\left(\left(\|\mathbf{H}\|_{s+1, \tau_{i}}^{2}+\|\mathbf{H}\|_{s+1, q_{j}}^{2}\right)^{\frac{1}{2}}\left(\left\|P_{h}(\mathbf{e})\right\|_{0, \tau_{i}}^{2}+\left\|P_{h}(\mathbf{e})\right\|_{0, q_{j}}^{2}\right)^{\frac{1}{2}}+\right. \\
\left.\sum_{a_{i j} \in \mathscr{T}_{m}}\left(\|\mathbf{E}\|_{s+1, \tau_{i}}^{2}+\|\mathbf{E}\|_{s+1, q_{j}}^{2}\right)^{\frac{1}{2}}\left(\left\|P_{h}(\mathbf{h})\right\|_{0, \tau_{i}}^{2}+\left\|P_{h}(\mathbf{h})\right\|_{0, q_{j}}^{2}\right)^{\frac{1}{2}}\right)+ \\
\sum_{a_{i j} \in \mathscr{Q}_{m}}\left(\|\mathbf{H}\|_{s+1, \tau_{i}}\left\|P_{h}(\mathbf{e})\right\|_{0, \tau_{i}}+\|\mathbf{E}\|_{s+1, \tau_{i}}\left\|P_{h}(\mathbf{h})\right\|_{0, \tau_{i}}\right)+ \\
\left.\left.(\mathbf{e})\left\|_{0, q_{i}}+\right\| \mathbf{E}\left\|_{s+1, q_{i}}\right\| P_{h}(\mathbf{h}) \|_{0, q_{i}}\right)\right) .
\end{gathered}
$$

Finally, using the inequality $\sum_{l} m_{l} n_{l} \leq\left(\sum_{l} m_{l}^{2}\right)^{\frac{1}{2}}\left(\sum_{l} n_{l}^{2}\right)^{\frac{1}{2}}, \forall m_{l}, n_{l} \in \mathbb{R}^{+}$we deduce an upper bound for $b\left(P_{h}(\mathbf{W})-\mathbf{W}, P_{h}(\mathbf{w})\right)$ :

$$
\begin{aligned}
& b\left(P_{h}(\mathbf{W})-\mathbf{W}, P_{h}(\mathbf{w})\right) \\
\leq & C \xi_{h}\left(\|\mathbf{H}\|_{P H^{s+1}(\Omega)}\left\|P_{h}(\mathbf{e})\right\|_{0, \Omega}+\|\mathbf{E}\|_{P H^{s+1}(\Omega)}\left\|P_{h}(\mathbf{h})\right\|_{0, \Omega}\right) \\
\leq & C \xi_{h}\left(\|\mathbf{E}\|_{P H^{s+1}(\Omega)}^{2}+\|\mathbf{H}\|_{P H^{s+1}(\Omega)}^{2}\right)^{\frac{1}{2}}\left(\left\|P_{h}(\mathbf{e})\right\|_{0, \Omega}^{2}+\left\|P_{h}(\mathbf{h})\right\|_{0, \Omega}^{2}\right)^{\frac{1}{2}} .
\end{aligned}
$$

We can now consider again 59, , use the upper bounds for $a\left(P_{h}(\mathbf{W})-\mathbf{W}, P_{h}(\mathbf{w})\right)$ and $b\left(P_{h}(\mathbf{W})-\right.$ $\left.\mathbf{W}, P_{h}(\mathbf{w})\right)$ and, by introducing the notation $\|\mathbf{W}\|_{P H^{s+1}(\Omega)}=\left(\|\mathbf{E}\|_{P H^{s+1}(\Omega)}^{2}+\|\mathbf{H}\|_{P H^{s+1}(\Omega)}^{2}\right)^{\frac{1}{2}}$, we obtain:

$$
\begin{aligned}
& \underline{\zeta}\left(\left\|P_{h}(\mathbf{e}(t))\right\|_{0, \Omega}^{2}+\left\|P_{h}(\mathbf{h}(t))\right\|_{0, \Omega}^{2}\right) \\
\leq & \int_{0}^{t} C \xi_{h}\|\mathbf{W}(\varsigma)\|_{P H^{s+1}(\Omega)}\left(\left\|P_{h}(\mathbf{e}(\varsigma))\right\|_{0, \Omega}^{2}+\left\|P_{h}(\mathbf{h}(\varsigma))\right\|_{0, \Omega}^{2}\right)^{\frac{1}{2}} d \varsigma .
\end{aligned}
$$

Now, we recall that:

$$
\|\mathbf{W}\|_{\mathcal{C}^{0}\left(\left[0, t_{f}\right], P H^{s+1}(\Omega)\right)}=\sup _{t \in\left[0, t_{f}\right]}\left(\|\mathbf{W}(t)\|_{P H^{s+1}(\Omega)}\right)=\max _{t \in\left[0, t_{f}\right]}\left(\|\mathbf{W}(t)\|_{P H^{s+1}(\Omega)}\right) .
$$

$\operatorname{RR} n^{\circ} 8257$ 
Let:

$$
\begin{aligned}
\Lambda(t) & =\left\|P_{h}(\mathbf{e}(t))\right\|_{0, \Omega}^{2}+\left\|P_{h}(\mathbf{h}(t))\right\|_{0, \Omega}^{2}=\left\|P_{h}(\mathbf{E}(t))-\mathbf{E}_{h}(t)\right\|_{0, \Omega}^{2} \\
& +\left\|P_{h}(\mathbf{H}(t))-\mathbf{H}_{h}(t)\right\|_{0, \Omega}^{2} .
\end{aligned}
$$

We deduce from 62 :

$$
\begin{aligned}
\Lambda(t) & \leq C \xi_{h} \int_{0}^{t}\|\mathbf{W}(\varsigma)\|_{P H^{s+1}(\Omega)} \Lambda(\varsigma)^{\frac{1}{2}} d \varsigma \\
& \leq C \xi_{h} \int_{0}^{t_{f}}\|\mathbf{W}(\varsigma)\|_{P H^{s+1}(\Omega)} \Lambda(\varsigma)^{\frac{1}{2}} d \varsigma, \quad \forall t \in\left[0, t_{f}\right] .
\end{aligned}
$$

Thus:

$$
\begin{aligned}
\max _{t \in\left[0, t_{f}\right]}(\Lambda(t)) & \leq C \xi_{h} \int_{0}^{t_{f}}\|\mathbf{W}(\varsigma)\|_{P H^{s+1}(\Omega)} \Lambda(\varsigma)^{\frac{1}{2}} d \varsigma \\
& \leq C \xi_{h} \int_{0}^{t_{f}}\|\mathbf{W}\|_{\mathcal{C}^{0}\left(\left[0, t_{f}\right], P H^{s+1}(\Omega)\right)} \max _{\varsigma \in\left[0, t_{f}\right]}\left(\Lambda(\varsigma)^{\frac{1}{2}}\right) d \varsigma .
\end{aligned}
$$

Since $\max _{t \in\left[0, t_{f}\right]}\left(\Lambda(t)^{\frac{1}{2}}\right)=\left[\max _{t \in\left[0, t_{f}\right]}(\Lambda(t))\right]^{\frac{1}{2}}$, we have the upper bound:

$$
\begin{aligned}
& {\left[\max _{t \in\left[0, t_{f}\right]}\left(\left\|P_{h}(\mathbf{E}(t))-\mathbf{E}_{h}(t)\right\|_{0, \Omega}^{2}+\left\|P_{h}(\mathbf{H}(t))-\mathbf{H}_{h}(t)\right\|_{0, \Omega}^{2}\right)\right]^{\frac{1}{2}} } \\
\leq & C \xi_{h} t_{f}\|\mathbf{W}\|_{\mathcal{C}^{0}\left(\left[0, t_{f}\right], P H^{s+1}(\Omega)\right)},
\end{aligned}
$$

and because:

$$
\begin{aligned}
& \left\|P_{h}(\mathbf{W}(t))-\mathbf{W}_{h}(t)\right\|_{0, \Omega} \\
= & \left(\left\|P_{h}(\mathbf{E}(t))-\mathbf{E}_{h}(t)\right\|_{0, \Omega}^{2}+\left\|P_{h}(\mathbf{H}(t))-\mathbf{H}_{h}(t)\right\|_{0, \Omega}^{2}\right)^{\frac{1}{2}},
\end{aligned}
$$

we finally obtain:

$$
\max _{t \in\left[0, t_{f}\right]}\left(\left\|P_{h}(\mathbf{W}(t))-\mathbf{W}_{h}(t)\right\|_{0, \Omega}\right) \leq C \xi_{h} t_{f}\|\mathbf{W}\|_{\mathcal{C}^{0}\left(\left[0, t_{f}\right], P H^{s+1}(\Omega)\right)} \cdot
$$

Corollary 1. Under the hypotheses of Theorem 1, there exists a generic constant $C>0$ independent of $h$ such that the error $\mathbf{w}=\mathbf{W}-\mathbf{W}_{h}$ satisfies the following a priori estimation:

$$
\|\mathbf{w}\|_{\mathcal{C}^{0}\left(\left[0, t_{f}\right], L^{2}(\Omega)\right)} \leq C \xi_{h} t_{f}\|\mathbf{W}\|_{\mathcal{C}^{0}\left(\left[0, t_{f}\right], P H^{s+1}(\Omega)\right)} .
$$

Proof. Applying Minkowski's inequality we have:

$$
\begin{aligned}
\|\mathbf{w}\|_{0, \Omega}=\left\|\mathbf{W}-\mathbf{W}_{h}\right\|_{0, \Omega} & =\left\|\mathbf{W}-P_{h}(\mathbf{W})+P_{h}(\mathbf{W})-\mathbf{W}_{h}\right\|_{0, \Omega} \\
& \leq\left\|\mathbf{W}-P_{h}(\mathbf{W})\right\|_{0, \Omega}+\left\|P_{h}(\mathbf{W})-\mathbf{W}_{h}\right\|_{0, \Omega} .
\end{aligned}
$$

But, using again the inequalities 45-47) (with $r=0$ ) of Lemma 2 and for $h_{\tau}$ and $h_{q}$ near 0 , 
we get:

$$
\begin{aligned}
& \left\|\mathbf{W}-P_{h}(\mathbf{W})\right\|_{0, \Omega}^{2} \\
= & \sum_{i=1}^{N_{\tau}}\left\|\mathbf{W}-P_{h}(\mathbf{W})\right\|_{0, \tau_{i}}^{2}+\sum_{i=1}^{N_{q}}\left\|\mathbf{W}-P_{h}(\mathbf{W})\right\|_{0, q_{i}}^{2} \\
\leq & \sum_{i=1}^{N_{\tau}}\left(C h_{\tau_{i}}^{\min \{s, p\}+1}\|\mathbf{W}\|_{s+1, \tau_{i}}\right)^{2}+\sum_{i=1}^{N_{q}}\left(C h_{q_{i}}^{\min \{s, k\}+1}\|\mathbf{W}\|_{s+1, q_{i}}\right)^{2} \\
\leq & \sum_{i=1}^{N_{\tau}}\left(C h_{\tau}^{\min \{s, p\}+1}\|\mathbf{W}\|_{s+1, \tau_{i}}\right)^{2}+\sum_{i=1}^{N_{q}}\left(C h_{q}^{\min \{s, k\}+1}\|\mathbf{W}\|_{s+1, q_{i}}\right)^{2} \\
\leq & \sum_{i=1}^{N_{\tau}}\left(C h_{\tau}^{\min \{s, p\}}\|\mathbf{W}\|_{s+1, \tau_{i}}\right)^{2}+\sum_{i=1}^{N_{q}}\left(C h_{q}^{\min \{s, k\}}\|\mathbf{W}\|_{s+1, q_{i}}\right)^{2} \\
\leq & \left(C \xi_{h}\right)^{2}\left(\sum_{i=1}^{N_{\tau}}\|\mathbf{W}\|_{s+1, \tau_{i}}^{2}+\sum_{i=1}^{N_{q}}\|\mathbf{W}\|_{s+1, q_{i}}^{2}\right) \\
\leq & \left(C \xi_{h}\right)^{2}\|\mathbf{W}\|_{P H^{s+1}(\Omega)}^{2} .
\end{aligned}
$$

We thus obtain:

$$
\left\|\mathbf{W}-P_{h}(\mathbf{W})\right\|_{0, \Omega} \leq C \xi_{h}\|\mathbf{W}\|_{P H^{s+1}(\Omega)} \leq C t_{f} \xi_{h}\|\mathbf{W}\|_{P H^{s+1}(\Omega)},
$$

and we conclude that:

$$
\|\mathbf{w}\|_{\mathcal{C}^{0}\left(\left[0, t_{f}\right], L^{2}(\Omega)\right)} \leq C \xi_{h} t_{f}\|\mathbf{W}\|_{\mathcal{C}^{0}\left(\left[0, t_{f}\right], P H^{s+1}(\Omega)\right)}
$$

In summary, we obtain that for the semi-discrete scheme the error is of order $\mathcal{O}\left(\xi_{h}\right)$.

\subsubsection{Convergence of the fully discrete problem}

The fully discrete scheme (14)- 15 can be seen as the time discretization of a system of ordinary differential equations. The global formulation 42 yields:

$$
m\left(\partial_{t} \mathbf{W}_{h}(t), \mathbf{T}^{\prime}\right)=-\left[a\left(\mathbf{W}_{h}(t), \mathbf{T}^{\prime}\right)+b\left(\mathbf{W}_{h}(t), \mathbf{T}^{\prime}\right)\right], \quad \forall \mathbf{T}^{\prime} \in V_{h}^{6}
$$

The latter equation can be evaluated at the time stations $t_{n}$ and $t_{n+\frac{1}{2}}$ from which we deduce:

$$
\begin{aligned}
& m\left(\left(\begin{array}{c}
\partial_{t} \mathbf{E}_{h}\left(t_{n+\frac{1}{2}}\right) \\
\partial_{t} \mathbf{H}_{h}\left(t_{n}\right)
\end{array}\right), \mathbf{T}^{\prime}\right) \\
= & -\left[a\left(\left(\begin{array}{c}
\mathbf{E}_{h}\left(t_{n}\right) \\
\mathbf{H}_{h}\left(t_{n+\frac{1}{2}}\right)
\end{array}\right), \mathbf{T}^{\prime}\right)+b\left(\left(\begin{array}{c}
\mathbf{E}_{h}\left(t_{n}\right) \\
\mathbf{H}_{h}\left(t_{n+\frac{1}{2}}\right)
\end{array}\right), \mathbf{T}^{\prime}\right)\right] .
\end{aligned}
$$

When time integration relies on the LF2 scheme, the fully discrete scheme can be rewritten as:

$$
\begin{aligned}
& m\left(\left(\begin{array}{c}
\mathbf{E}_{h}^{n+1} \\
\mathbf{H}_{h}^{n+\frac{1}{2}}
\end{array}\right), \mathbf{T}^{\prime}\right) \\
&=m\left(\left(\begin{array}{c}
\mathbf{E}_{h}^{n} \\
\mathbf{H}_{h}^{n-\frac{1}{2}}
\end{array}\right), \mathbf{T}^{\prime}\right)-\Delta t\left[a\left(\left(\begin{array}{c}
\mathbf{E}_{h}^{n} \\
\mathbf{H}_{h}^{n+\frac{1}{2}}
\end{array}\right), \mathbf{T}^{\prime}\right)+b\left(\left(\begin{array}{c}
\mathbf{E}_{h}^{n} \\
\mathbf{H}_{h}^{n+\frac{1}{2}}
\end{array}\right), \mathbf{T}^{\prime}\right)\right] .
\end{aligned}
$$


In other words:

$$
\begin{aligned}
& m\left(\left(\begin{array}{c}
\mathbf{E}_{h}^{n+1} \\
\mathbf{H}_{h}^{n+\frac{1}{2}}
\end{array}\right), \mathbf{T}^{\prime}\right) \\
= & m\left(\left(\begin{array}{c}
\mathbf{E}_{h}^{n} \\
\mathbf{H}_{h}^{n-\frac{1}{2}}
\end{array}\right), \mathbf{T}^{\prime}\right)+\Delta t m\left(\left(\begin{array}{c}
S_{h}^{m a g}\left(\mathbf{H}_{h}^{n+\frac{1}{2}}\right) \\
S_{h}^{e l}\left(\mathbf{E}_{h}^{n}\right)
\end{array}\right), \mathbf{T}^{\prime}\right) .
\end{aligned}
$$

Analoguously, in the case of the LF4 time integration, the fully discrete scheme writes:

$$
\begin{aligned}
& m\left(\left(\begin{array}{c}
\mathbf{E}_{h}^{n+1} \\
\mathbf{H}_{h}^{n+\frac{1}{2}}
\end{array}\right), \mathbf{T}^{\prime}\right) \\
= & m\left(\left(\begin{array}{c}
\mathbf{E}_{h}^{n} \\
\mathbf{H}_{h}^{n-\frac{1}{2}}
\end{array}\right), \mathbf{T}^{\prime}\right)+ \\
& \Delta t m\left(\left(\begin{array}{c}
S_{h}^{\text {mag }}\left(\mathbf{H}_{h}^{n+\frac{1}{2}}\right) \\
S_{h}^{e l}\left(\mathbf{E}_{h}^{n}\right)
\end{array}\right), \mathbf{T}^{\prime}\right)+ \\
& \frac{\Delta t^{3}}{24} m\left(\left(\begin{array}{c}
S_{h}^{\text {mag }} \circ S_{h}^{e l} \circ S_{h}^{\text {mag }}\left(\mathbf{H}_{h}^{n+\frac{1}{2}}\right) \\
S_{h}^{e l} \circ S_{h}^{\text {mag }} \circ S_{h}^{e l}\left(\mathbf{E}_{h}^{n}\right)
\end{array}\right), \mathbf{T}^{\prime}\right) .
\end{aligned}
$$

We first study the consistency error of the scheme. For this, we define $\widehat{\mathbf{E}}_{h}^{n+1}$ and $\widehat{\mathbf{H}}_{h}^{n+\frac{1}{2}}$ by:

- In the case of the LF2 time intgretaion:

$$
\begin{aligned}
& m\left(\left(\begin{array}{c}
\widehat{\mathbf{E}}_{h}^{n+1} \\
\widehat{\mathbf{H}}_{h}^{n+\frac{1}{2}}
\end{array}\right), \mathbf{T}^{\prime}\right) \\
= & m\left(\left(\begin{array}{c}
\mathbf{E}_{h}\left(t_{n}\right) \\
\mathbf{H}_{h}\left(t_{n-\frac{1}{2}}\right)
\end{array}\right), \mathbf{T}^{\prime}\right)- \\
& \Delta t\left[a\left(\left(\begin{array}{c}
\mathbf{E}_{h}\left(t_{n}\right) \\
\mathbf{H}_{h}\left(t_{n+\frac{1}{2}}\right)
\end{array}\right), \mathbf{T}^{\prime}\right)+b\left(\left(\begin{array}{c}
\mathbf{E}_{h}\left(t_{n}\right) \\
\mathbf{H}_{h}\left(t_{n+\frac{1}{2}}\right)
\end{array}\right), \mathbf{T}^{\prime}\right)\right] .
\end{aligned}
$$

- In the case of the LF4 time integration:

$$
\begin{aligned}
& m\left(\left(\begin{array}{c}
\widehat{\mathbf{E}}_{h}^{n+1} \\
\widehat{\mathbf{H}}_{h}^{n+\frac{1}{2}}
\end{array}\right), \mathbf{T}^{\prime}\right) \\
& =m\left(\left(\begin{array}{c}
\mathbf{E}_{h}\left(t_{n}\right) \\
\mathbf{H}_{h}\left(t_{n+\frac{1}{2}}\right)
\end{array}\right), \mathbf{T}^{\prime}\right)+\Delta t m\left(\left(\begin{array}{c}
S_{h}^{m a g}\left(\mathbf{H}_{h}\left(t_{n+\frac{1}{2}}\right)\right) \\
S_{h}^{\text {el }}\left(\mathbf{E}_{h}\left(t_{n}\right)\right)
\end{array}\right), \mathbf{T}^{\prime}\right)+ \\
& \frac{\Delta t^{3}}{24} m\left(\left(\begin{array}{c}
S_{h}^{\text {mag }} \circ S_{h}^{e l} \circ S_{h}^{\text {mag }}\left(\mathbf{H}_{h}\left(t_{n+\frac{1}{2}}\right)\right) \\
S_{h}^{e l} \circ S_{h}^{\text {mag }} \circ S_{h}^{e l}\left(\mathbf{E}_{h}\left(t_{n}\right)\right)
\end{array}\right), \mathbf{T}^{\prime}\right) .
\end{aligned}
$$

Then, $\left(\left\|\widehat{\mathbf{E}}_{h}^{n+1}-\mathbf{E}_{h}\left(t_{n+1}\right)\right\|_{0, \Omega}^{2}+\left\|\widehat{\mathbf{H}}_{h}^{n+\frac{1}{2}}-\mathbf{H}_{h}\left(t_{n+\frac{1}{2}}\right)\right\|_{0, \Omega}^{2}\right)^{\frac{1}{2}}$ stands for the consistency error.

Our goal is now to find an upper bound for the latter that will depend on the time step $\Delta t$. By substracting $m\left(\left(\begin{array}{c}\mathbf{E}_{h}\left(t_{n+1}\right) \\ \mathbf{H}_{h}\left(t_{n+\frac{1}{2}}\right)\end{array}\right), \mathbf{T}^{\prime}\right)$ to 68 , and using 64 , we get: 
- In the case of the LF2 time scheme:

$$
\begin{aligned}
& m\left(\left(\begin{array}{c}
\widehat{\mathbf{E}}_{h}^{n+1}-\mathbf{E}_{h}\left(t_{n+1}\right) \\
\widehat{\mathbf{H}}_{h}^{n+\frac{1}{2}}-\mathbf{H}_{h}\left(t_{n+\frac{1}{2}}\right)
\end{array}\right), \mathbf{T}^{\prime}\right) \\
= & m\left(\left(\begin{array}{c}
\mathbf{E}_{h}\left(t_{n}\right)-\mathbf{E}_{h}\left(t_{n+1}\right) \\
\mathbf{H}_{h}\left(t_{n-\frac{1}{2}}\right)-\mathbf{H}_{h}\left(t_{n+\frac{1}{2}}\right)
\end{array}\right), \mathbf{T}^{\prime}\right)+\Delta t m\left(\left(\begin{array}{c}
\partial_{t} \mathbf{E}_{h}\left(t_{n+\frac{1}{2}}\right) \\
\partial_{t} \mathbf{H}_{h}\left(t_{n}\right)
\end{array}\right), \mathbf{T}^{\prime}\right) .
\end{aligned}
$$

- In the case of the LF4 time scheme:

$$
\begin{aligned}
& m\left(\left(\begin{array}{c}
\widehat{\mathbf{E}}_{h}^{n+1}-\mathbf{E}_{h}\left(t_{n+1}\right) \\
\widehat{\mathbf{H}}_{h}^{n+\frac{1}{2}}-\mathbf{H}_{h}\left(t_{n+\frac{1}{2}}\right)
\end{array}\right), \mathbf{T}^{\prime}\right) \\
= & m\left(\left(\begin{array}{c}
\mathbf{E}_{h}\left(t_{n}\right)-\mathbf{E}_{h}\left(t_{n+1}\right) \\
\mathbf{H}_{h}\left(t_{n-\frac{1}{2}}\right)-\mathbf{H}_{h}\left(t_{n+\frac{1}{2}}\right)
\end{array}\right), \mathbf{T}^{\prime}\right)+\Delta t m\left(\left(\begin{array}{c}
S_{h}^{\text {mag }}\left(\mathbf{H}_{h}\left(t_{n+\frac{1}{2}}\right)\right) \\
S_{h}^{e l}\left(\mathbf{E}_{h}\left(t_{n}\right)\right)
\end{array}\right), \mathbf{T}^{\prime}\right)+ \\
& \frac{\Delta t^{3}}{24} m\left(\left(\begin{array}{c}
S_{h}^{\text {mag }} \circ S_{h}^{e l} \circ S_{h}^{\text {mag }}\left(\mathbf{H}_{h}\left(t_{n+\frac{1}{2}}\right)\right) \\
S_{h}^{e l} \circ S_{h}^{\text {mag }} \circ S_{h}^{e l}\left(\mathbf{E}_{h}\left(t_{n}\right)\right)
\end{array}\right), \mathbf{T}^{\prime}\right) .
\end{aligned}
$$

Since:

$$
\partial_{t} \mathbf{E}_{h}\left(t_{n+\frac{1}{2}}\right)=S_{h}^{\text {mag }}\left(\mathbf{H}_{h}\left(t_{n+\frac{1}{2}}\right)\right) \text { and } \partial_{t} \mathbf{H}_{h}\left(t_{n}\right)=S_{h}^{e l}\left(\left(\mathbf{E}_{h}\left(t_{n}\right)\right)\right),
$$

one deduces that:

$$
\begin{aligned}
& m\left(\left(\begin{array}{c}
\widehat{\mathbf{E}}_{h}^{n+1}-\mathbf{E}_{h}\left(t_{n+1}\right) \\
\widehat{\mathbf{H}}_{h}^{n+\frac{1}{2}}-\mathbf{H}_{h}\left(t_{n+\frac{1}{2}}\right)
\end{array}\right), \mathbf{T}^{\prime}\right) \\
= & m\left(\left(\begin{array}{c}
\mathbf{E}_{h}\left(t_{n}\right)-\mathbf{E}_{h}\left(t_{n+1}\right) \\
\mathbf{H}_{h}\left(t_{n-\frac{1}{2}}\right)-\mathbf{H}_{h}\left(t_{n+\frac{1}{2}}\right)
\end{array}\right), \mathbf{T}^{\prime}\right)+\Delta t m\left(\left(\begin{array}{c}
\partial_{t} \mathbf{E}_{h}\left(t_{n+\frac{1}{2}}\right) \\
\partial_{t} \mathbf{H}_{h}\left(t_{n}\right)
\end{array}\right), \mathbf{T}^{\prime}\right)+ \\
& \frac{\Delta t^{3}}{24} m\left(\left(\begin{array}{c}
\partial_{t}^{3} \mathbf{E}_{h}\left(t_{n+\frac{1}{2}}\right) \\
\partial_{t}^{3} \mathbf{H}_{h}\left(t_{n}\right)
\end{array}\right), \mathbf{T}^{\prime}\right) .
\end{aligned}
$$

Then, by using Taylor-Lagrange expansions and assuming that $\mathbf{W} \in \mathcal{C}^{3}\left(\left[0, t_{f}\right],\left(L^{2}(\Omega)\right)^{6}\right)($ respectively $\left.\mathbf{W} \in \mathcal{C}^{5}\left(\left[0, t_{f}\right],\left(L^{2}(\Omega)\right)^{6}\right)\right)$ in the LF2 case (respectively in the LF4 case), we find:

- In the case of the LF2 time scheme:

$$
\left(\left\|\widehat{\mathbf{E}}_{h}^{n+1}-\mathbf{E}_{h}\left(t_{n+1}\right)\right\|_{0, \Omega}^{2}+\left\|\widehat{\mathbf{H}}_{h}^{n+\frac{1}{2}}-\mathbf{H}_{h}\left(t_{n+\frac{1}{2}}\right)\right\|_{0, \Omega}^{2}\right)^{\frac{1}{2}} \leq C \Delta t^{3}\|\mathbf{W}\|_{\mathcal{C}^{3}\left(\left[0, t_{f}\right],\left(L^{2}(\Omega)\right)\right)} .
$$

- In the case of the LF4 time scheme:

$$
\left(\left\|\widehat{\mathbf{E}}_{h}^{n+1}-\mathbf{E}_{h}\left(t_{n+1}\right)\right\|_{0, \Omega}^{2}+\left\|\widehat{\mathbf{H}}_{h}^{n+\frac{1}{2}}-\mathbf{H}_{h}\left(t_{n+\frac{1}{2}}\right)\right\|_{0, \Omega}^{2}\right)^{\frac{1}{2}} \leq C \Delta t^{5}\|\mathbf{W}\|_{\mathcal{C}^{5}\left(\left[0, t_{f}\right],\left(L^{2}(\Omega)\right)\right)} .
$$

We now look at the global error of the scheme. In other words, we aim at evaluating the quantity:

$$
\max _{n \in\left\{0, \ldots, n_{f}\right\}}\left(\left\|\mathbf{E}\left(t_{n}\right)-\mathbf{E}_{h}^{n}\right\|_{0, \Omega}^{2}+\left\|\mathbf{H}\left(t_{n-\frac{1}{2}}\right)-\mathbf{H}_{h}^{n-\frac{1}{2}}\right\|_{0, \Omega}^{2}\right)^{\frac{1}{2}} .
$$

We start by studying the term:

$$
\left\|\mathbf{E}_{h}\left(t_{n}\right)-\mathbf{E}_{h}^{n}\right\|_{0, \Omega}^{2}+\left\|\mathbf{H}_{h}\left(t_{n-\frac{1}{2}}\right)-\mathbf{H}_{h}^{n-\frac{1}{2}}\right\|_{0, \Omega}^{2}
$$

$\mathrm{RR} \mathrm{n}^{\circ} 8257$ 
- In the case of the LF2 time scheme, adding $m\left(\begin{array}{c}\mathbf{E}_{h}\left(t_{n+1}\right) \\ \mathbf{H}_{h}\left(t_{n+\frac{1}{2}}\right)\end{array}, \mathbf{T}^{\prime}\right)$ to 68 , we get:

$$
\begin{aligned}
& m\left(\left(\begin{array}{l}
\mathbf{E}_{h}\left(t_{n+1}\right)-\mathbf{E}_{h}\left(t_{n}\right) \\
\mathbf{H}_{h}\left(t_{n+\frac{1}{2}}\right)-\mathbf{H}_{h}\left(t_{n-\frac{1}{2}}\right)
\end{array}\right), \mathbf{T}^{\prime}\right) \\
= & -\Delta t\left[a\left(\left(\begin{array}{l}
\mathbf{E}_{h}\left(t_{n}\right) \\
\mathbf{H}_{h}\left(t_{n+\frac{1}{2}}\right)
\end{array}\right), \mathbf{T}^{\prime}\right)+b\left(\left(\begin{array}{l}
\mathbf{E}_{h}\left(t_{n}\right) \\
\mathbf{H}_{h}\left(t_{n+\frac{1}{2}}\right)
\end{array}\right), \mathbf{T}^{\prime}\right)\right]+ \\
& m\left(\left(\begin{array}{l}
\mathbf{E}_{h}\left(t_{n+1}\right)-\widehat{\mathbf{E}}_{h}^{n+1} \\
\mathbf{H}_{h}\left(t_{n+\frac{1}{2}}\right)-\widehat{\mathbf{H}}_{h}^{n+\frac{1}{2}}
\end{array}\right), \mathbf{T}^{\prime}\right) .
\end{aligned}
$$

- In the case of the LF4 time scheme, we obtain similarly:

$$
\begin{aligned}
& m\left(\left(\begin{array}{l}
\mathbf{E}_{h}\left(t_{n+1}\right)-\mathbf{E}_{h}\left(t_{n}\right) \\
\mathbf{H}_{h}\left(t_{n+\frac{1}{2}}\right)-\mathbf{H}_{h}\left(t_{n-\frac{1}{2}}\right)
\end{array}\right), \mathbf{T}^{\prime}\right) \\
= & \Delta t m\left(\left(\begin{array}{l}
S_{h}^{\text {mag }}\left(\mathbf{H}_{h}\left(t_{n+\frac{1}{2}}\right)\right) \\
\left.S_{h}^{\text {el }}\left(\mathbf{E}_{h}\left(t_{n}\right)\right), \mathbf{T}^{\prime}\right)+
\end{array}\right)\right. \\
& \frac{\Delta t^{3}}{24} m\left(\left(\begin{array}{l}
S_{h}^{\text {mag }} \circ S_{h}^{\text {el }} \circ S_{h}^{\text {mag }}\left(\mathbf{H}_{h}\left(t_{n+\frac{1}{2}}\right)\right) \\
S_{h}^{\text {el }} \circ S_{h}^{\text {mag }} \circ S_{h}^{e l}\left(\mathbf{E}_{h}\left(t_{n}\right)\right)
\end{array}\right), \mathbf{T}^{\prime}\right)+ \\
& m\left(\left(\begin{array}{l}
\mathbf{E}_{h}\left(t_{n+1}\right)-\widehat{\mathbf{E}}_{h}^{n+1} \\
\mathbf{H}_{h}\left(t_{n+\frac{1}{2}}\right)-\widehat{\mathbf{H}}_{h}^{n+\frac{1}{2}}
\end{array}\right), \mathbf{T}^{\prime}\right) .
\end{aligned}
$$

Setting $\mathfrak{e}_{h}^{n}=\mathbf{E}_{h}\left(t_{n}\right)-\mathbf{E}_{h}^{n}$ and $\mathfrak{h}_{h}^{n-\frac{1}{2}}=\mathbf{H}_{h}\left(t_{n-\frac{1}{2}}\right)-\mathbf{H}_{h}^{n-\frac{1}{2}}$, and substracting 67 to 72 on one hand, and 699 to 73 on the other hand, we can write:

- In the case of the LF2 time scheme:

$$
\begin{aligned}
& m\left(\left(\begin{array}{l}
\mathfrak{e}_{h}^{n+1} \\
\mathfrak{h}_{h}^{n+\frac{1}{2}}
\end{array}\right), \mathbf{T}^{\prime}\right) \\
= & m\left(\left(\begin{array}{l}
\mathfrak{e}_{h}^{n} \\
\mathfrak{h}_{h}^{n-\frac{1}{2}}
\end{array}\right), \mathbf{T}^{\prime}\right)-\Delta t\left[a\left(\left(\begin{array}{l}
\mathfrak{e}_{h}^{n} \\
\mathfrak{h}_{h}^{n+\frac{1}{2}}
\end{array}\right), \mathbf{T}^{\prime}\right)+b\left(\left(\begin{array}{l}
\mathfrak{e}_{h}^{n} \\
\mathfrak{h}_{h}^{n+\frac{1}{2}}
\end{array}\right), \mathbf{T}^{\prime}\right)\right]+ \\
& m\left(\left(\begin{array}{l}
\mathbf{E}_{h}\left(t_{n+1}\right)-\widehat{\mathbf{E}}_{h}^{n+1} \\
\mathbf{H}_{h}\left(t_{n+\frac{1}{2}}\right)-\widehat{\mathbf{H}}_{h}^{n+\frac{1}{2}}
\end{array}\right), \mathbf{T}^{\prime}\right) .
\end{aligned}
$$

- In the case of the LF4 time scheme:

$$
\begin{aligned}
& m\left(\left(\begin{array}{l}
\mathfrak{e}_{h}^{n+1} \\
\mathfrak{h}_{h}^{n+\frac{1}{2}}
\end{array}\right), \mathbf{T}^{\prime}\right) \\
= & m\left(\left(\begin{array}{l}
\mathfrak{e}_{h}^{n} \\
\mathfrak{h}_{h}^{n-\frac{1}{2}}
\end{array}\right), \mathbf{T}^{\prime}\right)+\Delta t m\left(\left(\begin{array}{c}
S_{h}^{m a g}\left(\mathfrak{h}_{h}^{n+\frac{1}{2}}\right) \\
S_{h}^{e l}\left(\mathfrak{e}_{h}^{n}\right)
\end{array}\right), \mathbf{T}^{\prime}\right)+ \\
& \frac{\Delta t^{3}}{24} m\left(\left(\begin{array}{l}
S_{h}^{m a g} \circ S_{h}^{e l} \circ S_{h}^{m a g}\left(\mathfrak{h}_{h}^{n+\frac{1}{2}}\right) \\
S_{h}^{e l} \circ S_{h}^{m a g} \circ S_{h}^{\text {el }}\left(\mathfrak{e}_{h}^{n}\right)
\end{array}\right), \mathbf{T}^{\prime}\right)+ \\
& m\left(\left(\begin{array}{l}
\mathbf{E}_{h}\left(t_{n+1}\right)-\widehat{\mathbf{E}}_{h}^{n+1} \\
\mathbf{H}_{h}\left(t_{n+\frac{1}{2}}\right)-\widehat{\mathbf{H}}_{h}^{n+\frac{1}{2}}
\end{array}\right), \mathbf{T}^{\prime}\right) .
\end{aligned}
$$


We now define the discrete energy:

$$
\mathfrak{N}^{n}=\sum_{i=1}^{N} \mathfrak{N}_{i}^{n}, \quad \text { with } \mathfrak{N}_{i}^{n}=\frac{1}{2}\left\|\mathfrak{e}_{i}^{n}\right\|_{\epsilon_{i}, c_{i}}^{2}+\frac{1}{2} \ll \mathfrak{h}_{i}^{n-\frac{1}{2}} \mathfrak{h}_{i}^{n+\frac{1}{2}} \gg_{\mu_{i}, c_{i}}
$$

where $\mathfrak{e}_{i}=\mathfrak{e}_{h \mid c_{i}}$ and $\mathfrak{h}_{i}=\left.\mathfrak{h}_{h}\right|_{c_{i}}$. Similarly to the stability analysis conducted in [20], we can show that under a CFL condition we have that:

- In the case of the LF2 time scheme:

$$
\left(\left\|\mathfrak{e}_{i}^{n}\right\|_{0, \Omega}^{2}+\left\|\mathfrak{h}_{i}^{n-\frac{1}{2}}\right\|_{0, \Omega}^{2}\right)^{\frac{1}{2}} \leq C \Delta t^{2}\|\mathbf{W}\|_{\mathcal{C}^{3}\left(\left[0, t_{f}\right], L^{2}(\Omega)\right)} .
$$

- In the case of the LF4 time scheme:

$$
\left(\left\|\mathfrak{e}_{i}^{n}\right\|_{0, \Omega}^{2}+\left\|\mathfrak{h}_{i}^{n-\frac{1}{2}}\right\|_{0, \Omega}^{2}\right)^{\frac{1}{2}} \leq C \Delta t^{4}\|\mathbf{W}\|_{\mathcal{C}^{5}\left(\left[0, t_{f}\right], L^{2}(\Omega)\right)} .
$$

In summary, the error resulting from the use of the second order (respectively fourth order) leap-frog scheme behaves as $\mathcal{O}\left(\Delta t^{2}\right)$ (respectively as $\mathcal{O}\left(\Delta t^{4}\right)$ ) and we can thus conclude using the triangular inequality together with Corollary 1 that the error for the fully discrete scheme is:

- In the case of the LF2 time scheme:

$$
\mathcal{O}\left(\Delta t^{2}\right)+\mathcal{O}\left(\xi_{h}\right)
$$

- In the case of the LF4 time scheme:

$$
\mathcal{O}\left(\Delta t^{4}\right)+\mathcal{O}\left(\xi_{h}\right)
$$

\section{$5 \quad$ Numerical and performance results}

In this section we present numerical results for the solution of the 2D Maxwell equations. More precisely, we consider the case of transverse magnetic waves:

$$
\left\{\begin{array}{l}
\mu \frac{\partial H_{x}}{\partial t} \quad+\frac{\partial E_{z}}{\partial y}=0 \\
\mu \frac{\partial H_{y}}{\partial t}-\frac{\partial E_{z}}{\partial x}=0 \\
\varepsilon \frac{\partial E_{z}}{\partial t}-\frac{\partial H_{y}}{\partial x}+\frac{\partial H_{x}}{\partial y}=0 .
\end{array}\right.
$$

The objectives of this section are to evaluate the effective convergence properties of the proposed DGTD- $\mathbb{P}_{p} \mathbb{Q}_{k}$ method by considering a test problem with sufficient regularity and for which an analytical solution is available one one hand, and to assess the computational peformances of the method on the other hand. Of course, since we are limiting ourselves to $2 \mathrm{D}$ problems, the latter objective will only be partially fulfilled because the most challenging problems, especially in terms of spatial discretization, are 3D ones. We will discuss further about this point in the conslusion section but for now we note that a detailed assessment of the performances of the DGTD- $\mathbb{P}_{p} \mathbb{Q}_{k}$ method for realistic 3D test problems will be the subject of a dedicated study. 


\subsection{Standing wave in a PEC square cavity}

We first consider a test problem for studying numerically the convergence properties of the proposed DGTD- $\mathbb{P}_{p} \mathbb{Q}_{k}$ method. We compute the evolution of the $(1,1)$ mode in a perfectly electrically conducting (PEC) square cavity $\Omega=[0,1 \mathrm{~m}] \times[0,1 \mathrm{~m}]$. The analytical solution is given by:

$$
\left\{\begin{aligned}
H_{x}(x, x, t) & =-\frac{\pi}{\omega} \sin (\pi x) \cos (\pi y) \sin (\omega t), \\
H_{y}(x, y, t) & =\frac{\pi}{\omega} \cos (\pi x) \sin (\pi y) \sin (\omega t), \\
E_{z}(x, y, t) & =\sin (\pi x) \sin (\pi y) \cos (\omega t),
\end{aligned}\right.
$$

where $\omega=2 \pi f$ is the angular frequency, with $f=212 \mathrm{MHz}$ in the present case. For this numerical convergence study we have used a sequence of 4 hybrid non-conforming meshes built from cartesian meshes of the unit square with resepctively $7 \times 7,13 \times 13,19 \times 19$ and $25 \times 25$ points. The two coarsest meshes are shown on Fig. 2. The $h$-wise convergence of several configurations
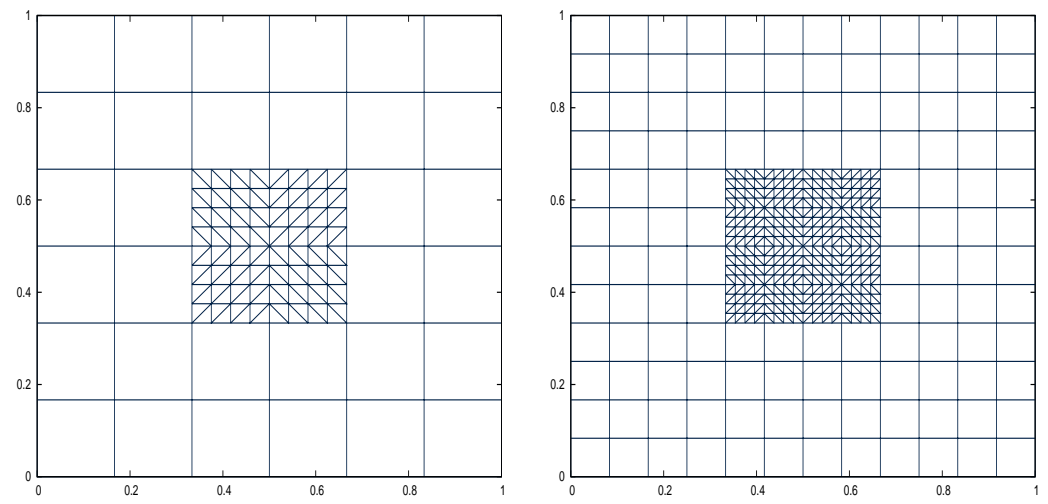

Figure 2: Standing wave in a square PEC cavity: two hybrid meshes used for the numerical convergence study.

of the DGTD- $\mathbb{P}_{p} \mathbb{Q}_{k}$ method is visualized on the graphs of figure 3. The graphs of the top (respectively, bottom) figure correspond to the DGTD-P $\mathbb{Q}_{k}$ method combined to the second order leap-frog (LF2) scheme (respectively, the fourth order leap-frog (LF4) scheme). We observe that in each case, taking into account the regularity of the solution, the obtained convergence orders match the theoretical expectations i.e. $\min \{2, p, k\}$ in the case of the LF2 scheme and $\min \{4, p, k\}$ in the case of the LF4 scheme. We complete this series of results by assessing the computational efficiency of the proposed DGTD-P $\mathbb{Q}_{k}$ method through the graphs of the error reduction in terms of the CPU time (see the plots of figure 4). Looking at the results for the DGTD- $\mathbb{P}_{1} \mathbb{Q}_{3}$ and DGTD- $\mathbb{P}_{1} \mathbb{Q}_{4}$ methods, we do not observe a notable reduction of the error when switching from the LF2 to the LF4 time scheme. The situation is totally different and clearly in favor of the LF4 time scheme (at least, for the present test problem) when considering the DGTD- $\mathbb{P}_{2} \mathbb{Q}_{3}$, DGTD- $\mathbb{P}_{2} \mathbb{Q}_{4}$ and DGTD- $\mathbb{P}_{3} \mathbb{Q}_{3}$ methods (recall that each point of the graphs correspond to a mesh, the rightmost points on each line always matching the coarsest mesh among the 4 selected hybrid meshes). The plots of figure 4 also allow a performance comparison for a given time scheme. For instance, for the LF2 scheme, we note that the DGTD-P $\mathbb{Q}_{3}$ method used with the hybrid mesh based on a $13 \times 13$ cartesian discretization, yields an error level which is about 6 times lower than the one obtained with the DGTD-P $\mathbb{Q}_{4}$ method applied with the $19 \times 19$ mesh, while the CPU time is reduced by a factor 2 . For the LF4 scheme, considering the 
DGTD- $\mathbb{P}_{4} \mathbb{Q}_{4}$ method used with the $13 \times 13$ mesh, the CPU reduction is about 2.5 for an error level lowered by a factor approximately equal to 50 , as compared to the DGTD- $\mathbb{P}_{3} \mathbb{Q}_{3}$ method applied with the $25 \times 25$ mesh.
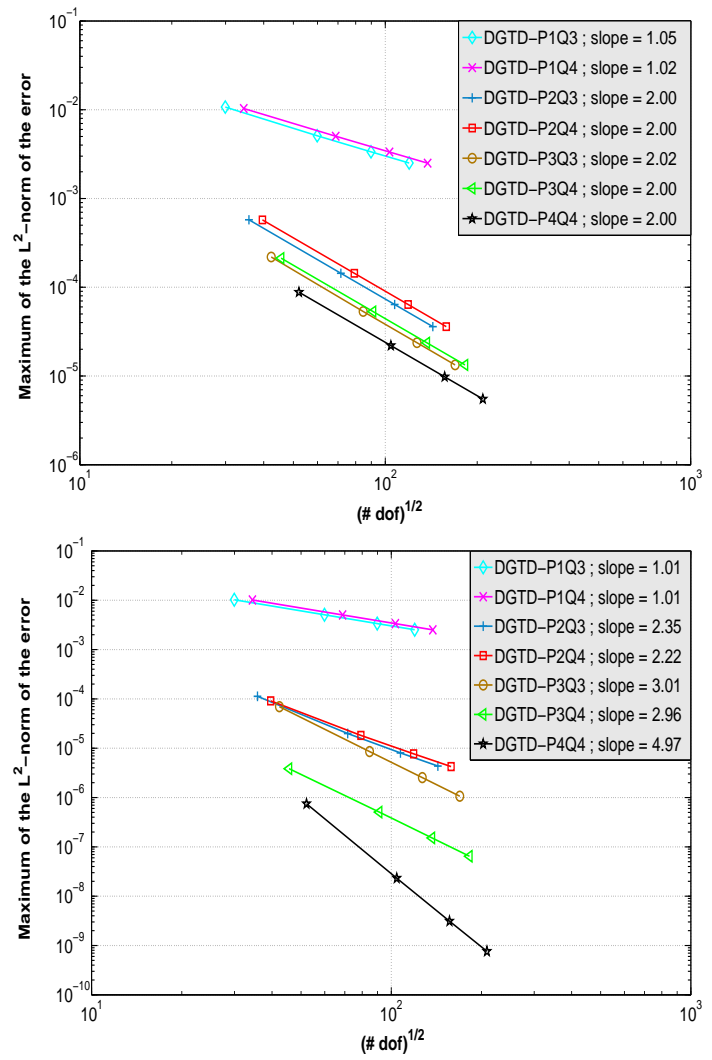

Figure 3: Standing wave in a square PEC cavity: numerical convergence in terms of mesh refinement (i.e. $h$-wise). Top: $\mathrm{DGTD} \mathbb{P}_{p} \mathbb{Q}_{k}$ method combined to the LF2 scheme. Bottom: DGTD-P $\mathbb{Q}_{k}$ method combined to the LF4 scheme.

\subsection{Scattering of plane wave by an airfoil profile}

The test problem that we consider now is the scattering of a plane wave by an airfoil profile. The computational domain is $\Omega=[-1 \mathrm{~m}, 2 \mathrm{~m}] \times[-1 \mathrm{~m}, 1 \mathrm{~m}]$. We impose the Silver-Müller absorbing condition on the rectangle delimiting the domain, while the surface of the airfoil is assumed to be a PEC boundary. The incident wave is defined as:

$$
\left\{\begin{array}{l}
E_{z, \text { inc }}\left(x_{1}, x_{2}, t\right)=\cos \left(\omega t-\kappa x_{1}\right), \\
H_{x, \text { inc }}\left(x_{1}, x_{2}, t\right)=0, \\
H_{y, \text { inc }}\left(x_{1}, x_{2}, t\right)=-\frac{\kappa}{\omega} \cos \left(\omega t-\kappa x_{1}\right),
\end{array}\right.
$$

where $\mathbf{k}=(\kappa, 0)^{\mathrm{T}}$ with $\kappa=\omega / c_{0}, \quad c_{0}$ being the wavespeed in vacuum and $\omega=2 \pi f$. The frequency is set to $f=600 \mathrm{MHz}$. We compare approximate solutions obtained using a fully 

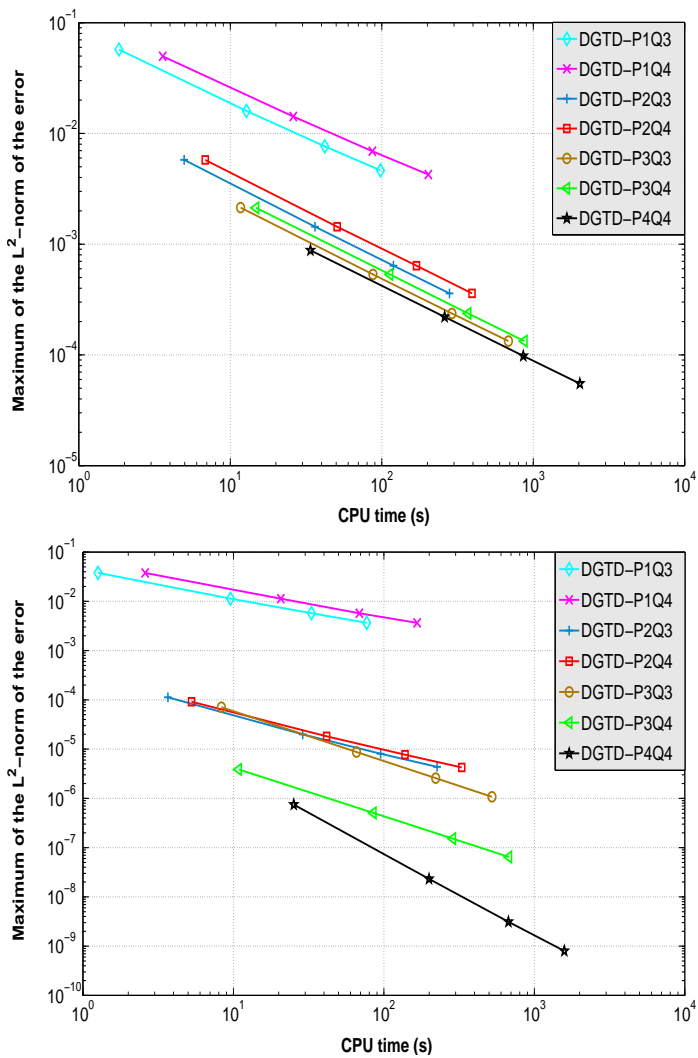

Figure 4: Standing wave in a square PEC cavity: computational efficiency in terms of mesh refinement (i.e. $h$-wise). Top: DGTD- $\mathbb{P}_{p} \mathbb{Q}_{k}$ method combined to the LF2 scheme. Bottom: DGTD- $\mathbb{P}_{p} \mathbb{Q}_{k}$ method combined to the LF4 scheme. 
triangular mesh (see figure 5 left) consisting of 84,444 elements, and a hybrid non-conforming mesh (see figure 5 right) consisting of 11,584 triangles and 172 quadrangles. Simulations are conducted for a physical time set to $1.67 \times 10^{-8} \mathrm{~s}$ corresponding to 10 periods of the incident wave oscillation. For this test problem, numerical results are presented for the DGTD-P $\mathbb{Q}_{k}$ method combined to the second order leap frog scheme.

For this test problem we do not have access to an analytical solution therefore we compare the approximate solutions resulting from various configurations of the DGTD-P $\mathbb{P}_{p}$ and DGTD-P $\mathbb{Q}_{k}$ methods. Firstly, we plot the time evolution of the $E_{z}$ component at two given points in the computational domain located near the airfoil profile in the triangular mesh or in the triangular part of the mesh in the case of the hybrid mesh. These time evolutions are shown on figure 6 . Secondly, we compute a discrete Fourier transform of the approximate solutions in time, on the fly during the last period of oscillation of the incident wave. Then, we visualize on figure 7 the contour lines of the real part of the $E_{z}$ component in the Fourier domain. Finally, performance results are given in tables 1 and 2. In particular, in table 2 we compare two ratios: the first one is the ratio of CPU times for two given configurations of the DGTD-P $\mathbb{P}_{p}$ and DGTD- $\mathbb{P}_{p} \mathbb{Q}_{k}$ methods, while the second one is the ratio of the total numbers of degrees of freedom. We note that, for the DGTD- $\mathbb{P}_{2}$ and DGTD- $\mathbb{P}_{1} \mathbb{Q}_{3}$ methods, the second ratio is close to 1 and the latter method is almost 4 times faster. Besides, on the plots of figure 6, it is rather difficult to make a distinction between these two methods. Comparing the DGTD-P $\mathbb{P}_{3}$ and DGTD-P $\mathbb{Q}_{3}$ methods, the ratio of CPU times is approaching 12 and the DGTD- $\mathbb{P}_{3}$ method requires more that 2 times more degrees of freedom. Similar remarks apply to the comparison of higher order configurations of both methods.
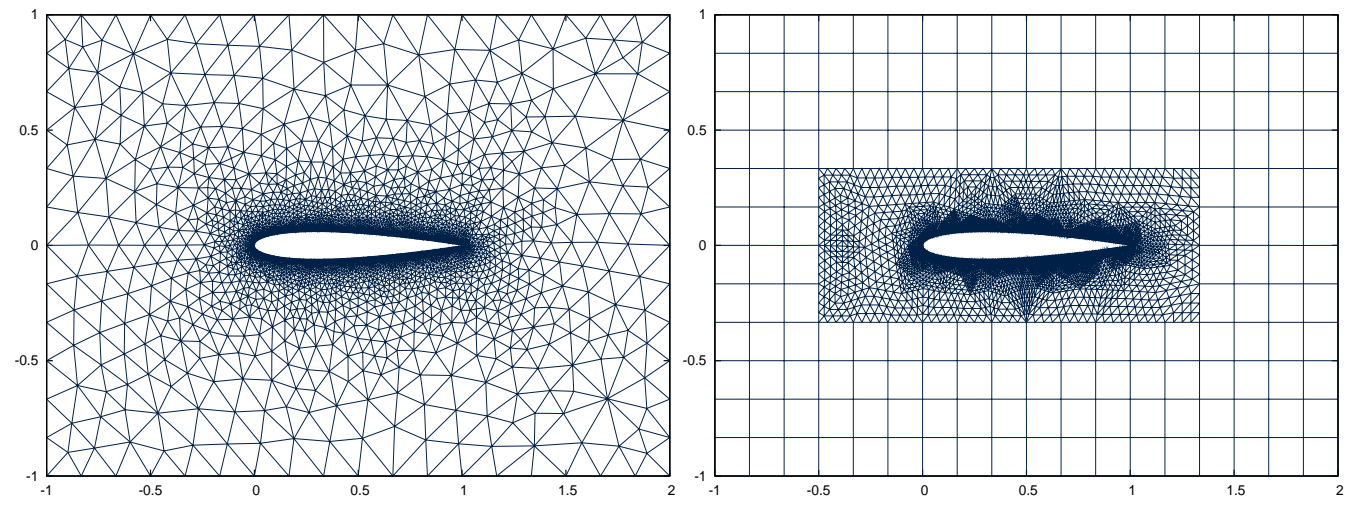

Figure 5: Scattering of plane wave by an airfoil profile: triangular and hybrid meshes.

\subsection{Scattering of plane wave by a dielectric cylinder}

We conclude this series of numerical experiments by considering the problem of scattering of a plane wave by a dielectric cylinder. The radius of the cylinder is $R=0.002 \mathrm{~m}$ and its relative ellectric permittivity is set to 7 . The cylinder is centered at the origin and the computational domain is articificially truncated by the square $\Omega=[-0.045 \mathrm{~m}, 0.045 \mathrm{~m}] \times[-0.045 \mathrm{~m}, 0.045 \mathrm{~m}]$. We impose the Silver-Müller absorbing condition on the square delimiting the domain. The 

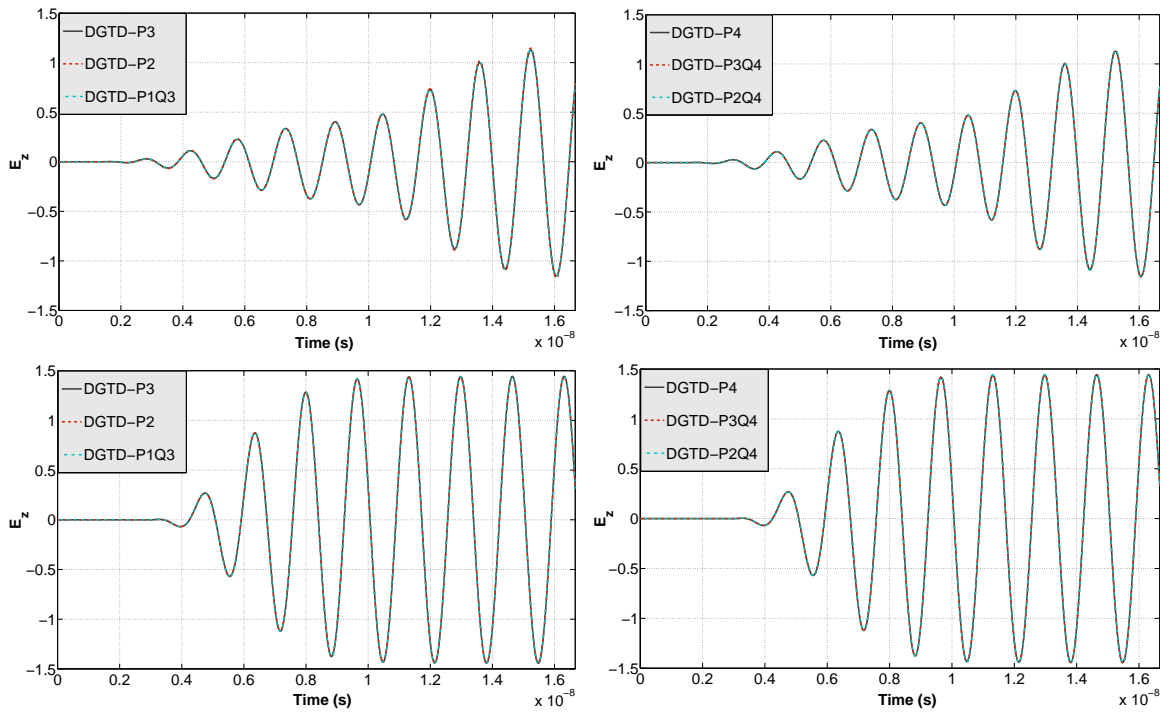

Figure 6: Scattering of plane wave by an airfoil profile: time evolution of the $E_{z}$ component at the points $(1.6,-0.6)$ (top plots) and $(-0.1,0.0)$ (bottom plots).

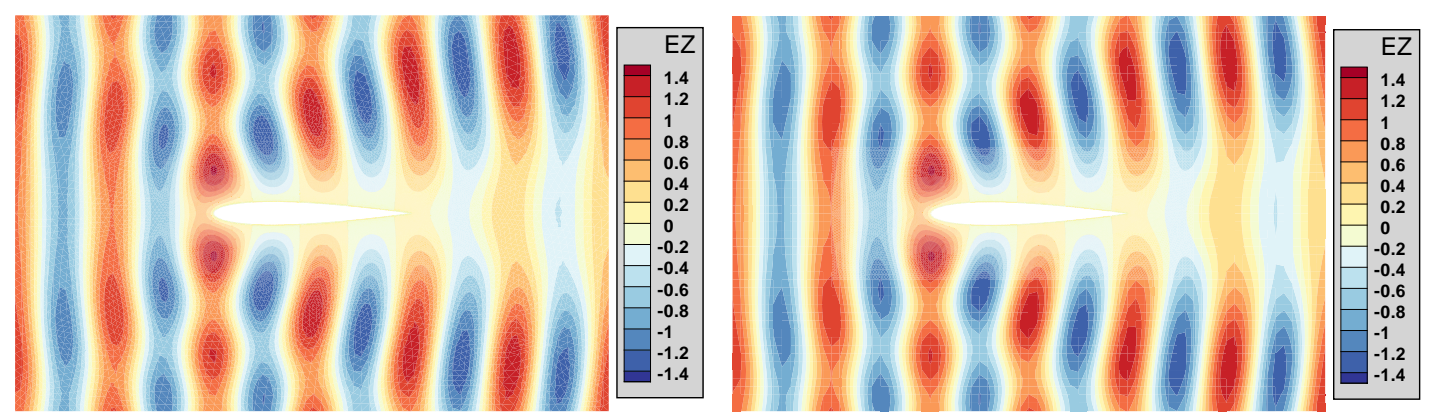

Figure 7: Scattering of plane wave by an airfoil profile: contour lines of the real part of the $E_{z}$ component in the Fourier domain. Left figure: DGTD- $\mathbb{P}_{4}$ method. Right figure: DGTD- $\mathbb{P}_{3} \mathbb{Q}_{4}$ method.

Table 1: Scattering of plane wave by an airfoil profile: CPU time and total number of degress of freedom for the various configurations of the DGTD- $\mathbb{P}_{p}$ and DGTD- $\mathbb{P}_{p} \mathbb{Q}_{k}$ methods.

\begin{tabular}{c|c|c|c} 
Mesh & Method & CPU time & $\#$ d.o.f \\
\hline \multirow{3}{*}{ Triangular } & DGTD- $_{2}$ & $132.8 \mathrm{~s}$ & 50,664 \\
& DGTD- $_{3}$ & $379.9 \mathrm{~s}$ & 84,440 \\
& DGTD- $_{4}$ & $1201.9 \mathrm{~s}$ & 126,660 \\
\hline \multirow{3}{*}{ Hybrid } & DGTD- $_{1} \mathbb{Q}_{3}$ & $32.5 \mathrm{~s}$ & 37,504 \\
& DGTD-P $_{2} \mathbb{Q}_{4}$ & $152.0 \mathrm{~s}$ & 73,804 \\
& DGTD- $\mathbb{P}_{3} \mathbb{Q}_{4}$ & $437.2 \mathrm{~s}$ & 120,140
\end{tabular}


Table 2: Scattering of plane wave by an airfoil profile: CPU time and total number of degress of freedom ratios for the various configurations of the DGTD- $\mathbb{P}_{p}$ and DGTD-P $\mathbb{Q}_{k}$ methods.

\begin{tabular}{|c|c|c|c|}
\hline \multicolumn{2}{|c|}{ Comparison } & & \\
\hline Method $(a)$ & Method (b) & $\mathrm{CPU}_{(b)}$ & $\#$ d.o.f $f_{(b)}$ \\
\hline DGTD-P $\mathbb{P}_{2}$ & DGTD- $\mathbb{P}_{1} \mathbb{Q}_{3}$ & 4.1 & 1.35 \\
\hline DGTD-P ${ }_{3}$ & DGTD- $\mathbb{P}_{1} \mathbb{Q}_{3}$ & 11.7 & 2.25 \\
\hline DGTD- $\mathbb{P}_{3}$ & DGTD- $\mathbb{P}_{2} \mathbb{Q}_{4}$ & 2.5 & 1.15 \\
\hline DGTD- $\mathbb{P}_{4}$ & DGTD- $\mathbb{P}_{1} \mathbb{Q}_{3}$ & 37.0 & 3.4 \\
\hline DGTD- $\mathbb{P}_{4}$ & DGTD- $\mathbb{P}_{2} \mathbb{Q}_{4}$ & 7.9 & 1.7 \\
\hline DGTD-P $_{4}$ & DGTD-P $_{3} \mathrm{Q}_{4}$ & 2.8 & 1.1 \\
\hline
\end{tabular}

incident wave is again defined as:

$$
\left\{\begin{array}{l}
E_{z, \text { inc }}\left(x_{1}, x_{2}, t\right)=\cos \left(\omega t-\kappa x_{1}\right) \\
H_{x, \text { inc }}\left(x_{1}, x_{2}, t\right)=0 \\
H_{y, \text { inc }}\left(x_{1}, x_{2}, t\right)=-\frac{\kappa}{\omega} \cos \left(\omega t-\kappa x_{1}\right),
\end{array}\right.
$$

where $\mathbf{k}=(\kappa, 0)^{\mathrm{T}}$ with $\kappa=\omega / c_{0}, \quad c_{0}$ being the wavespeed in vacuum (exterior of the cylinder) and $\omega=2 \pi f$. The frequency is set to $f=30 \mathrm{GHz}$. The relative permittivity of the inner cylinder is set to 7 while the relative permeability is set to 1 . This problem has an analytical solution which is expressed in terms of polar coordinates and special functions (see [1] for example). We compare approximate solutions obtained using a fully triangular mesh (see figure 8, top figures) consisting of 22,216 elements, and a hybrid non-conforming mesh (see figure 8 bottom figures) consisting of 7,128 triangles and 2,304 quadrangles. Simulations are conducted for a physical time set to $6.67 \times 10^{-10} \mathrm{~s}$ corresponding to 20 periods of the incident wave oscillation.

We again compare the approximate solutions resulting from various configurations of the DGTD$\mathbb{P}_{p}$ and DGTD- $\mathbb{P}_{p} \mathbb{Q}_{k}$ methods. As with the previous test problem, we compute a discrete Fourier transform of the approximate solutions in time, on the fly during the last period of oscillation of the incident wave. Then, we visualize on figures 10 and 11 the contour lines of the real part of the $E_{z}$ component in the Fourier domain. Note that on figure 11, we zoom in the area of the dielectric cylinder in order to better appreciate that the two configurations considered in these plots, i.e. the DGTD- $\mathbb{P}_{3}$ and $D G T D-P_{3} \mathbb{Q}_{2}$, yield almost identical solutions. All the selected methods are also compared on figure 9 in terms of the $1 \mathrm{D} x$-wise distribution of the real part of the $H_{y}$ and $E_{y}$. On these plots, we clearly observe that, for the triangular and hybrid nonconforming meshes used here, the DGTD- $\mathbb{P}_{3}$ and DGTD- $\mathbb{P}_{3} \mathbb{Q}_{2}$ methods give the best results. Finally, performance results are summarized in table 3 . We note that the DGTD- $\mathbb{P}_{3} \mathbb{Q}_{2}$ method is superior to the DGTD- $P_{3}$ method both from the point of view of accuracy and CPU time, with a reduction of the CPU time approaching 3.

\section{Conclusion}

In this paper, we have reported on our efforts to design a multi-element DGTD-P $\mathbb{Q}_{k}$ method on hybrid non-conforming meshes for solving the time-domain Maxwell equations. DGTD methods 

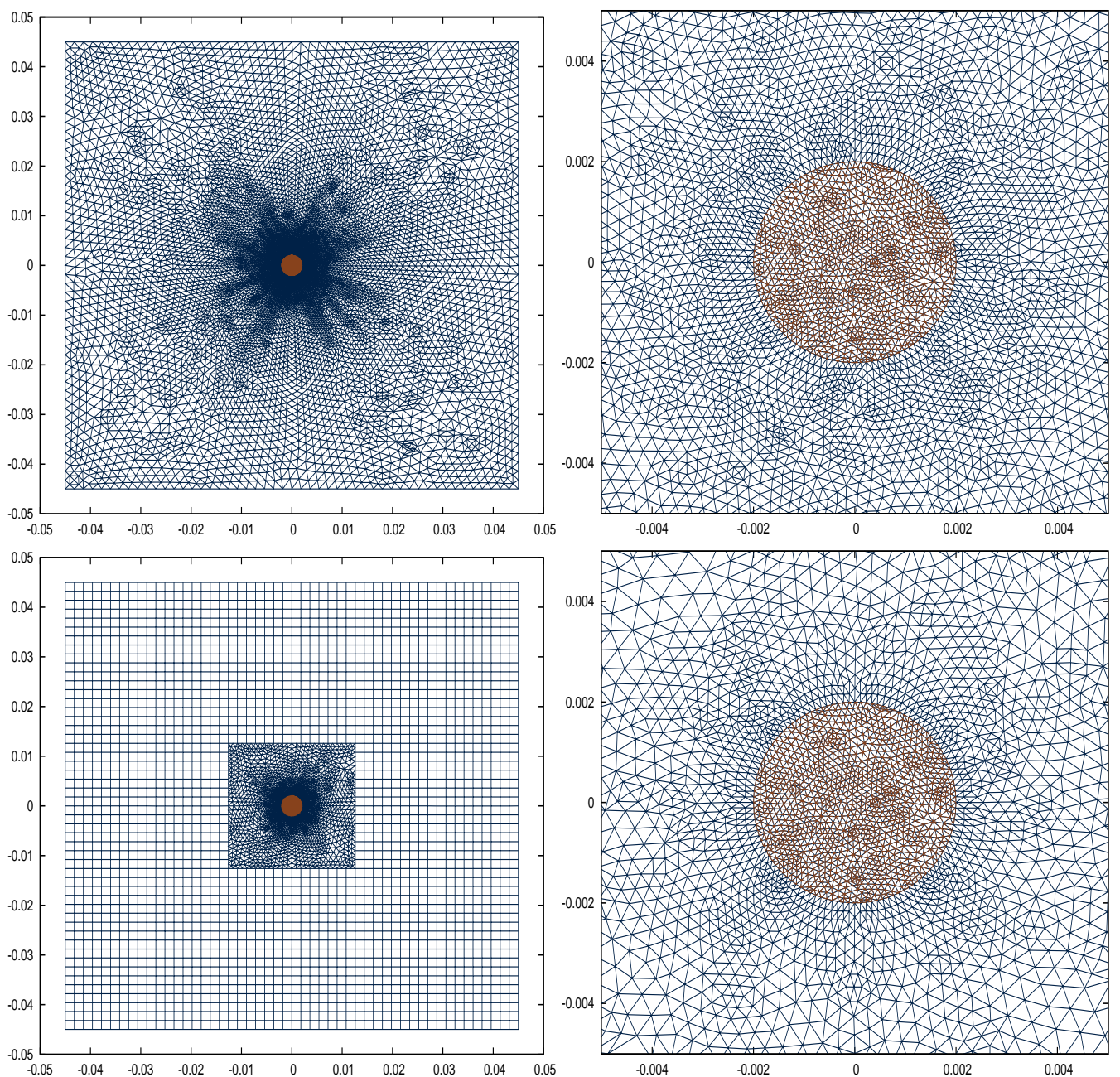

Figure 8: Scattering of plane wave by a dielectric cylinder: triangular and hybrid meshes.

Table 3: Scattering of plane wave by a dielectric cylinder: CPU time and total number of degress of freedom for the various configurations of the DGTD- $\mathbb{P}_{p}$ and DGTD- $\mathbb{P}_{p} \mathbb{Q}_{k}$ methods.

\begin{tabular}{c|c|c|c|c} 
Mesh & Method & CPU time & \# d.o.f & $L^{2}$ error \\
\hline \multirow{2}{*}{ Triangular } & DGTD- $\mathbb{P}_{2}$ & $391.1 \mathrm{~s}$ & 133,296 & $2.40 \times 10^{-2}$ \\
& DGTD- $_{3}$ & $1351.0 \mathrm{~s}$ & 222,160 & $6.21 \times 10^{-3}$ \\
\hline \multirow{2}{*}{ Hybrid } & DGTD- $_{2} \mathbb{Q}_{2}$ & $165.6 \mathrm{~s}$ & 63,504 & $1.86 \times 10^{-2}$ \\
& DGTD- $_{3} \mathbb{Q}_{2}$ & $458.7 \mathrm{~s}$ & 92,016 & $4.88 \times 10^{-3}$
\end{tabular}



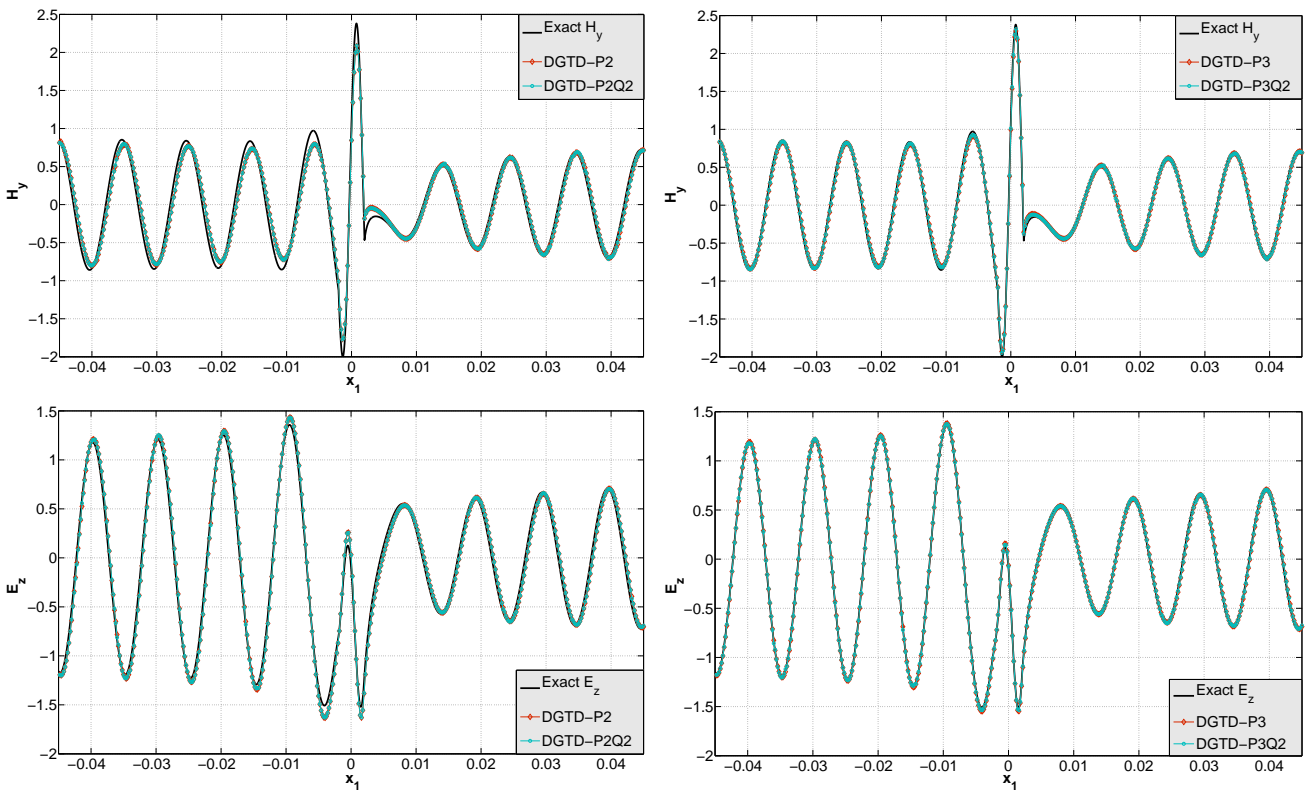

Figure 9: Scattering of plane wave by a dielectric cylinder: 1D $x$-wise distribution of the real part of the $H_{y}$ (top plots) and $E_{z}$ (bottom plots) components for $y=0$.
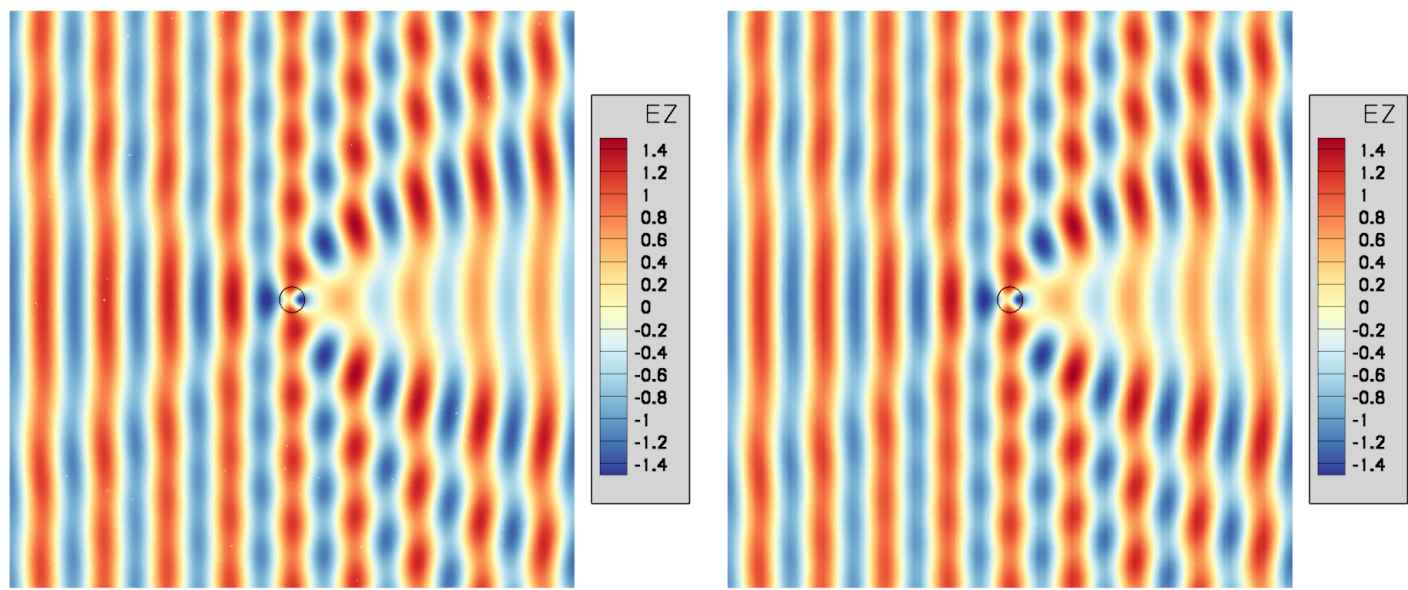

Figure 10: Scattering of plane wave by a dielectric cylinder: contour lines of the real part of the $E_{z}$ component in the Fourier domain. Left figure: DGTD- $\mathbb{P}_{3}$ method. Right figure: DGTD- $\mathbb{P}_{3} \mathbb{Q}_{2}$ method. 

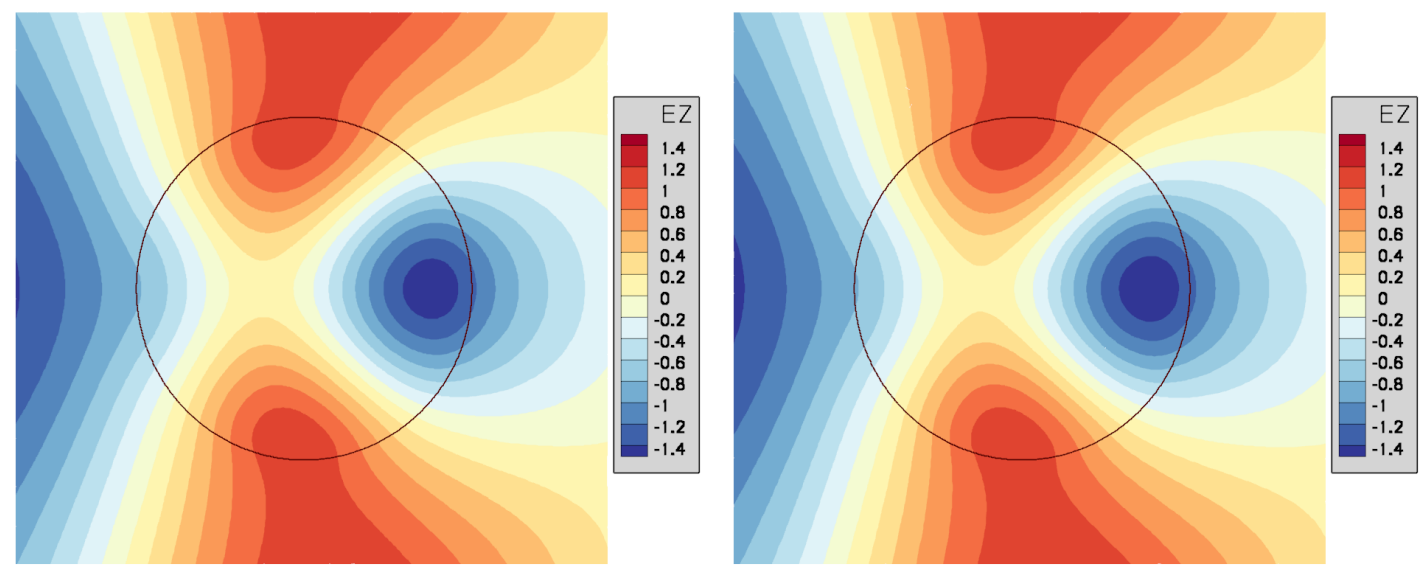

Figure 11: Scattering of plane wave by a dielectric cylinder: contour lines of the real part of the $E_{z}$ component in the Fourier domain (zoom in the area of the dielectric cylinder). Left figure: analytical solution. Right figure: DGTD- $\mathbb{P}_{3} \mathbb{Q}_{2}$ method.

have been extensively studied in the last decade for this purpose and have reached a level of maturity that made them applicable to realistic 3D problems. Noteworthy, for time-domain electromagnetics, the DGTD method has been selected as teh base method in the recently launched time-domain version of the well known HFSS commercial software 22]. Despite these advances, the exploitation of non-conforming unstructured meshes in the context of a DG-based numerical methodology applied to linear wave propagation PDE models, has been the subject of a few studies so far. Two remarkable conributions are those of Dosopoulos et al. [19 for 3D time-domain electromagnetics, and Hermann et al. 12 for 2D time-domain geoseismics. In the former work, non-conforming tetrahedral meshes are considered in the framework of an interior penalty DGTD method, and the parallelization of the resulting numerical methodology is discussed in detail. In the latter work, an ADER-DG method is formulated on non-conforming quadrangular-triangular meshes, and the method is also adapted to parallel computing platform. Here, we have presented a non-dissipative DGTD-P $\mathbb{Q}_{k}$ method formulated on non-conforming structured-unstructured meshes. The structured part of the mesh is assumed to rely on orthogonal hexahedra, while a general tetrahedral discretization is adopted in the unstructured part. The stability and the a priori convergence of the resulting DGTD- $\mathbb{P}_{p} \mathbb{Q}_{k}$ method have been investigated in the general 3D case. Numerical results have been discussed for 2D problems only. The present work can be seen as a complementary contribution demonstrating the benefits of relaxing the conformity requirement in the discretization process in view of facilitating the construction of computational meshes for complex propagation configurations, and improving the overall computational efficiency of the simulations. Since the numerical experiments discussed in this paper are limited to 2D problems, these two aspects have been addressed partially and the obtained results, albeit promising, need to be consolidated by considering realistic 3D problems. The implementation and parallelization of the proposed multi-element DGTD- $\mathbb{P}_{p} \mathbb{Q}_{k}$ method in the $3 \mathrm{D}$ case is underway [15] and will be detailed in a subsequent paper.

Acknowledgements. The authors gratefully acknowledge support from Région Ile-de-France in the framework of the MIEL3D-MESHER project of the System@tic Paris-Région cluster. 


\section{References}

[1] W. Cai and S. Deng. An upwinding embedded boundary method for Maxwell's equations in media with material interfaces: 2D case. J. Comput. Phys., 190(1):159-183, 2003.

[2] P. Ciarlet. The Finite Element Method for Elliptic Problems. North Holland-Elsevier Science Publisher, 1978.

[3] G. Cohen, X. Ferriéres, and S. Pernet. A spatial high order hexahedral discontinuous Galerkin method to solve Maxwell's equations in time-domain. J. Comput. Phys., 217(2):340-363, 2006.

[4] R.W. Davies, K. Morgan, and O. Hassan. A high order hybrid finite element method applied to the solution of electromagnetic wave scattering problems in the time domain. Comput. Mech., 44:321-331, 2009.

[5] S. Dosopoulos and J. F. Lee. Interior penalty discontinuous Galerkin finite element method for the time-dependent first order Maxwell's equations. IEEE Trans. Antennas and Propag., 58(12):4085-4090, 2010.

[6] H. Fahs. Development of a $h p$-like discontinuous Galerkin time-domain method on nonconforming simplicial meshes for electromagnetic wave propagation. Int. J. Numer. Anal. Mod., 6:193-216, 2009.

[7] H. Fahs and S. Lanteri. A high-order non-conforming discontinuous Galerkin method for time-domain electromagnetics. J. Comput. Appl. Math., 234:1088-1096, 2010.

[8] X. Ferrieres, J.-P. Parmantier, S. Bertuol, and A. R. Ruddle. Application of a hybrid finite difference/finite volume method to solve an automotive EMC problem. IEEE Trans. on Eletromag. Compatibility, 46(4):624-634, 2004.

[9] L. Fezoui, S. Lanteri, S. Lohrengel, and S. Piperno. Convergence and stability of a discontinuous Galerkin time-domain method for the 3D heterogeneous Maxwell equations on unstructured meshes. ESAIM: Math. Model. Numer. Anal., 39(6):1149-1176, 2005.

[10] S.G. Garcia, M.F. Pantoja, C.M. de Jong van Coevorden, A.R. Bretones, and R.G. Martin. Hybridizing DGTD and FDTD. In International Conference on Electromagnetics in Advanced Applications, pages 364-366. IEEE, 2007.

[11] S.G. Garcia, M.F. Pantoja, C.M. de Jong van Coevorden, A.R. Bretones, and R.G. Martin. A new hybrid DGTD/FDTD method in 2-D. IEEE Microw. Wireless Compon. Lett., 18(12):764-766, 2008.

[12] V. Hermann, M. Käser, and C.E. Castro. Non-conforming hybrid meshes for efficient 2-D wave propagation using the Discontinuous Galerkin Method. Geophys. J. Int., 184:746-758, 2011.

[13] J.S. Hesthaven and T. Warburton. Nodal high-order methods on unstructured grids. I. Time-domain solution of Maxwell's equations. J. Comput. Phys., 181(1):186-221, 2002.

[14] M. König, K. Busch, and J. Niegemann. The discontinuous Galerkin time-domain method for Maxwell's equations with anisotropic materials. Photonics and Nanostructures - Fundamentals and Applications, 8(4):303-309, 2010. 
[15] R. Léger, C. Durochat, and S. Lanteri. A high-order 3D multi-element Discontinuous Galerkin method for the time-domain Maxwell equations. In 9th International Symposium on Electric and Magnetic Fields (EMF 2013), Bruges, Belgium, 2013.

[16] E. Montseny, S. Pernet, X. Ferriéres, and G. Cohen. Dissipative terms and local timestepping improvements in a spatial high order discontinuous Galerkin scheme for the timedomain Maxwell's equations. J. Comput. Phys., 227(14):6795-6820, 2008.

[17] J. Niegemann, M. König, K. Stannigel, and K. Busch and. Higher-order time-domain methods for the analysis of nano-photonic systems. Photonics and Nanostructures - Fundamentals and Applications, 7(1):2-11, 2009.

[18] S. Pernet and X. Ferrieres. Hp a-priori error estimates for a non-dissipative spectral discontinuous Galerkin method to solve the Maxwell equations in the time domain. Math. Comput., 76:1801-1832, 2007.

[19] B. Zhao S. Dosopoulos and J. F. Lee. Non-conformal and parallel discontinuous Galerkin time domain method for Maxwell's equations: EM analysis of IC packages. J. Comput. Phys., 238(1):48-70, 2013.

[20] C. Scheid and S. Lanteri. Convergence of a Discontinuous Galerkin scheme for the mixed time domain Maxwell's equations in dispersive media. IMA J. Numer. Anal., 2012.

[21] S. Schnepp, E. Gjonaj, and T. Weiland. A hybrid finite integration. J. Comput. Phys., 229(11):4075-4096, 2010.

[22] H. Songoro, M. Vogel, and Z. Cendes. Keeping time with Maxwell's equations. IEEE Microw. Mag., 11(2):42-49, 2010.

[23] K. Stannigel, M. König, J. Niegemann, and K. Busch. Discontinuous Galerkin time-domain computations of metallic nanostructures. Optics Express, 17(17):14934-14947, 2009.

[24] A. Taflove and S.C. Hagness. Computational electrodynamics: the finite-difference timedomain method - 3rd ed. Artech House Publishers, 2005. 


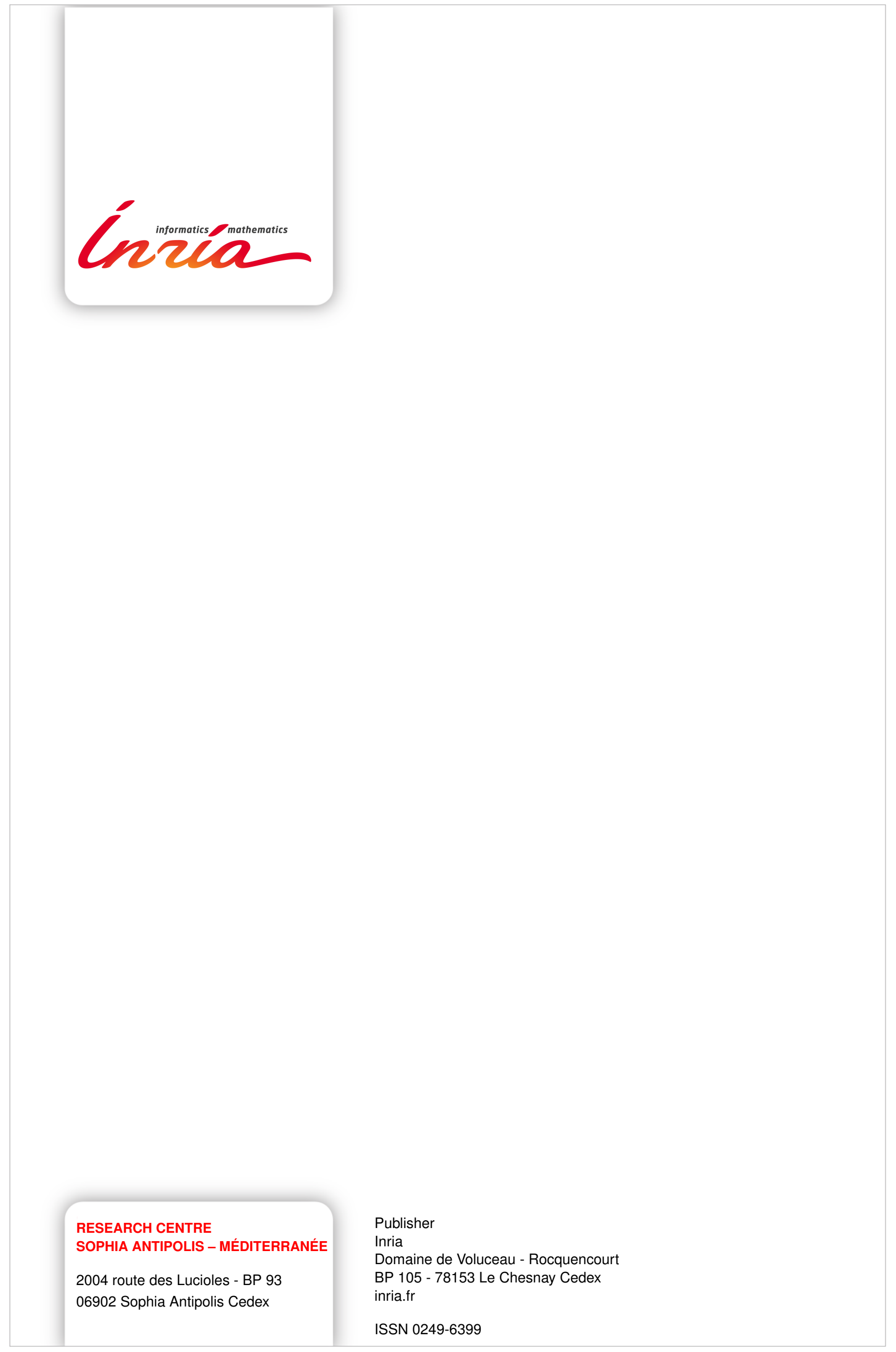

